\title{
2006
}

Lawrence Livermore National Laboratory Annual Illness and Injury Surveillance Report

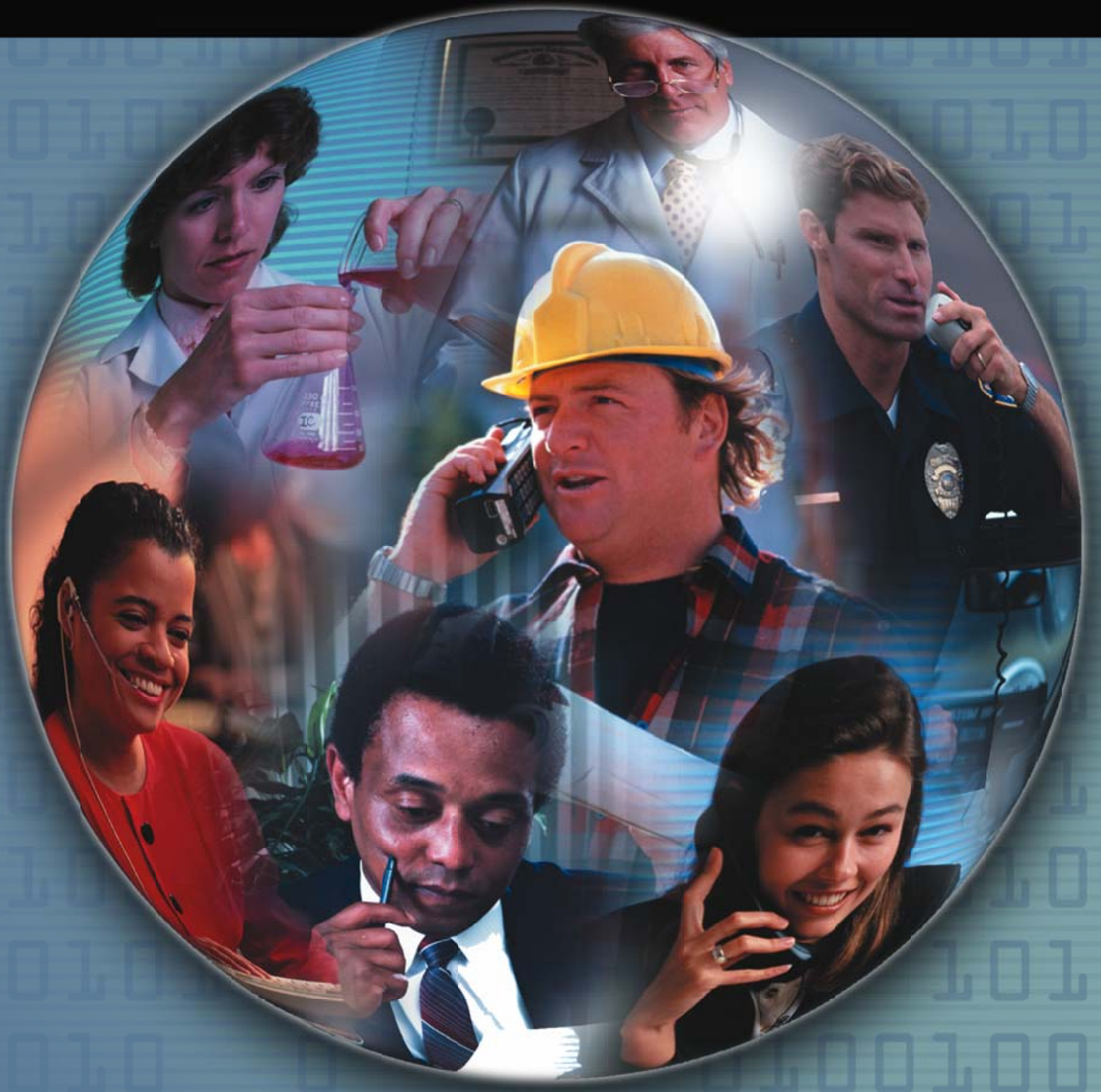




\section{Lawrence Livermore National Laboratory 2006 Illness and Injury Surveillance Report}

Questions or comments about this report or the Illness and Injury Surveillance Program (IISP) may be directed to:

E-mail:

Dr. Cliff Strader at cliff.strader@hq.doe.gov

or Dr. Bonnie Richter at bonnie.richter@hq.doe.gov

or direct letters to:

Mail Stop HS-13 / 270CC

U.S. Department of Energy

1000 Independence Avenue, S.W.

Washington, DC 20585-0270

Additional information about the Department of Energy's Office of Illness and Injury Prevention Programs, the IISP, and annual reports for DOE sites participating in this program can be found at:

http://www.hss.energy.gov/healthsafety/WSHP/epi/surv/

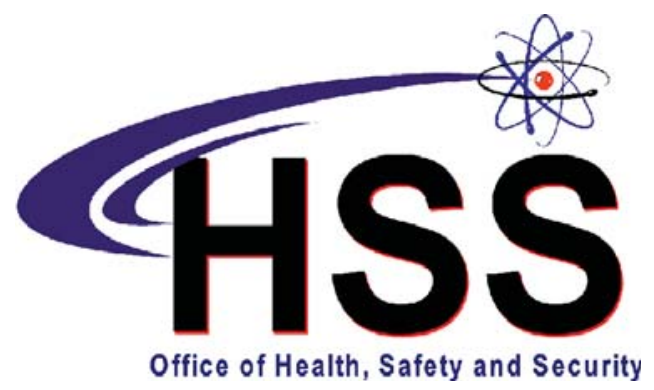

ACKNOWLEDGEMENT

LifeART images copyright 2000 Lippincott Williams \& Wilkins. All rights reserved.

This document was produced under contract number DE-AC05-06OR23100 between the

U.S. Department of Energy and Oak Ridge Associated Universities. 


\title{
Lawrence Livermore National Laboratory 2006 Illness and Injury Surveillance Report
}

\author{
At A Glance
}

A total of 9,044 LLNL employees were included in illness and injury surveillance in 2006; 2,817 (31 percent) women and 6,227 (69 percent) men.

The 233 absences among 214 women resulted in an absence rate of 8.3 per 100 workers $(233 / 2,817)$. The 396 absences reported by 372 men resulted in an absence rate of 6.4 per 100 workers $(396 / 6,227)$. Absence rates have increased since 2003 for both men and women.

The average length of absences in 2006 was 22 days among men and 28 days among women. The average has decreased since 2004 among women but has been more stable among men since 2002 .

Among men, Crafts and Service workers had the highest absence rate, 10.3 and 10.0 per 100 workers, respectively. Among women, Crafts and Security and Fire workers had the highest absence rates, 29.4 and 16.2 per 100 workers, respectively. These high rates do not appear to be a new phenomenon. Since 2004, absence rates for Crafts and Security and Fire workers have been among the highest for women. Among men, Service workers were among the top two categories in absence rates every year since 2003 .

Women lost 6,542 calendar days due to illness and injury. Respiratory diseases (16.7 percent), genitourinary conditions (14.9 percent), and musculoskeletal disorders (14.2 percent) accounted for 45.8 percent of all reported diagnoses among women.

Men lost 5,543 calendar days due to illness and injury. Injuries (19.3 percent), musculoskeletal disorders (18.4 percent), and respiratory diseases (15.1 percent), accounted for 52.8 percent of all reported diagnoses among men.

Since 2002, respiratory and musculoskeletal conditions were among the top 3 diagnostic categories reported by women. Injuries were consistently among the top 3 categories for men.

There were 64 OSHA-recordable events among women and 108 OSHA-recordable events among men. The rate of workers with an OSHA event was similar for women and men $(2.3$ and 1.7 per 100 workers, respectively). Since 2003, the rates have held steady or declined, a pattern similar to what we have seen across the DOE Complex, where OSHA rates have been declining since 1995.

Among women, the OSHA-recordable rates were highest among Crafts workers and among men highest among Security and Fire workers. 
Men aged 40 years and older and women 50 years and older tended to have higher OSHArecordable rates. Eighty-six percent of the OSHA health conditions involved musculoskeletal conditions or injuries.

Of the 160 OSHA events for which the type of accident was reported, 51.6 percent of the events for women were the result of repetitive trauma; among men, 41.8 percent were due to overexertion and strenuous movements. The conditions reported by women that were the result of repetitive trauma included mainly joint disorders (pain, stiffness, swelling, etc.). Only 3 carpal tunnel diagnoses were reported in this group; all were among women in the Professional and Administrative Support job categories. 
The Lawrence Livermore National

Laboratory Work Force - 2006

The Work Force by Gender and Age ....

The Work Force by Gender and Job

Category ......................................... 1

\section{Number and Length of Absences}

Absence Rate by Gender and Age .2

Number of Days Absent by

Gender and Age

Absence Rate by Job Category

and Gender

Average Duration of Absence by

Job Category and Gender.

\section{Diagnostic Categories}

Number of Diagnoses and Lost Calendar

Days by Diagnostic Category

(Categorized by ICD-9-CM) and Gender......4

Common Diagnoses Among Female

Workers in 2006 .5

Common Diagnoses Among Male

Workers in 2006

Number of Most Frequently Reported

Diagnoses by Job Category and Gender ......7

\section{Rates of Disease Occurrence}

Rates for All Illnesses and Injuries

Combined by Job Category, Gender,

and Age

Rates for Selected Diagnostic Categories

by Job Category, Gender, and Age. ..8

\section{Time Trends}

Age-Adjusted Rates for All Diagnoses

Combined Among Women and Men

from 2003 to 2006
Age-Adjusted Rates for Selected Diagnostic Categories Among Women and Men from

2003 to 2006

Age-Adjusted Rates for All Diagnoses

Combined Among Women and Men by

Job Category from 2003 to 2006 .... 12

\section{Sentinel Health Events for Occupations (SHEOs)}

Characteristics of SHEOs by Gender.... 13

SHEO Diagnoses by Gender 13

Occupational Safety and Health Administration (OSHA)-Recordable Events

OSHA-Recordable Events by Gender and Age 14

OSHA-Recordable Events by Job

Category and Gender 14

Diagnostic and Accident Categories for OSHA-Recordable Events

OSHA-Recordable Diagnoses by

Diagnostic Category and Gender .... 15

OSHA-Recordable Accidents by Type and Gender. 16

\section{Rates of OSHA-Recordable Events}

OSHA-Recordable Rates by Age and Job Categories Among Women, All

Diagnoses Combined 17

OSHA-Recordable Rates by Age and

Job Categories Among Men, All

Diagnoses Combined 17

Time Trends for OSHA-Recordable Events

Age-Adjusted Rates for All OSHA-Recordable Diagnoses Combined Among Women and Men by Job Category from 2003 to $2006 . . .18$

\section{Appendices}

Appendices A-W. 20 
The Lawrence Livermore National Laboratory Work Force - 2006

Figure 1. The Work Force by Gender and Age

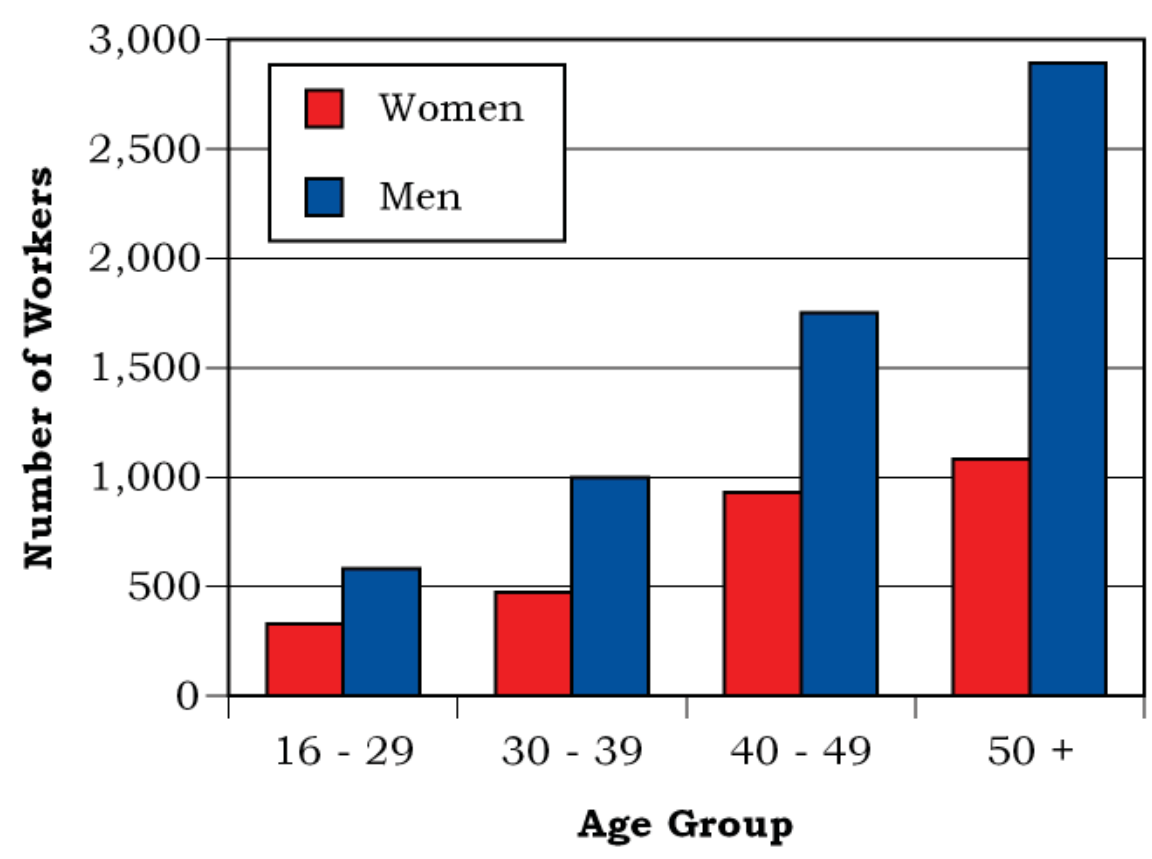

Figure 2. The Work Force by Gender and Job Category

\begin{tabular}{|l|c|c|}
\hline \multirow{2}{*}{ Job Category } & Women & Men \\
\hline \multirow{2}{*}{ Professional } & $\mathbf{5 7 1}$ & $\mathbf{2 , 7 1 0}$ \\
& $20 \%$ & $\mathbf{4 4 \%}$ \\
\hline \multirow{2}{*}{ Administrative Support } & $\mathbf{1 , 7 8 1}$ & $\mathbf{1 , 7 6 2}$ \\
& $\mathbf{6 3} \%$ & $\mathbf{2 8} \%$ \\
\hline \multirow{2}{*}{ Technical Support } & 315 & $\mathbf{8 4 0}$ \\
& $11 \%$ & $13 \%$ \\
\hline \multirow{2}{*}{ Service } & 96 & 220 \\
& $4 \%$ & $4 \%$ \\
\hline \multirow{2}{*}{ Security and Fire } & 37 & 326 \\
\multirow{2}{*}{ Crafts } & $1 \%$ & $\mathbf{5} \%$ \\
\hline \multirow{2}{*}{ Line Operators } & 17 & 369 \\
& $1 \%$ & $6 \%$ \\
\hline \multirow{2}{*}{ Total } & 0 & 0 \\
& $0 \%$ & $\mathbf{0} \%$ \\
\hline
\end{tabular}


Number and Length of Absences

Figure 3. Absence Rate by Gender and Age

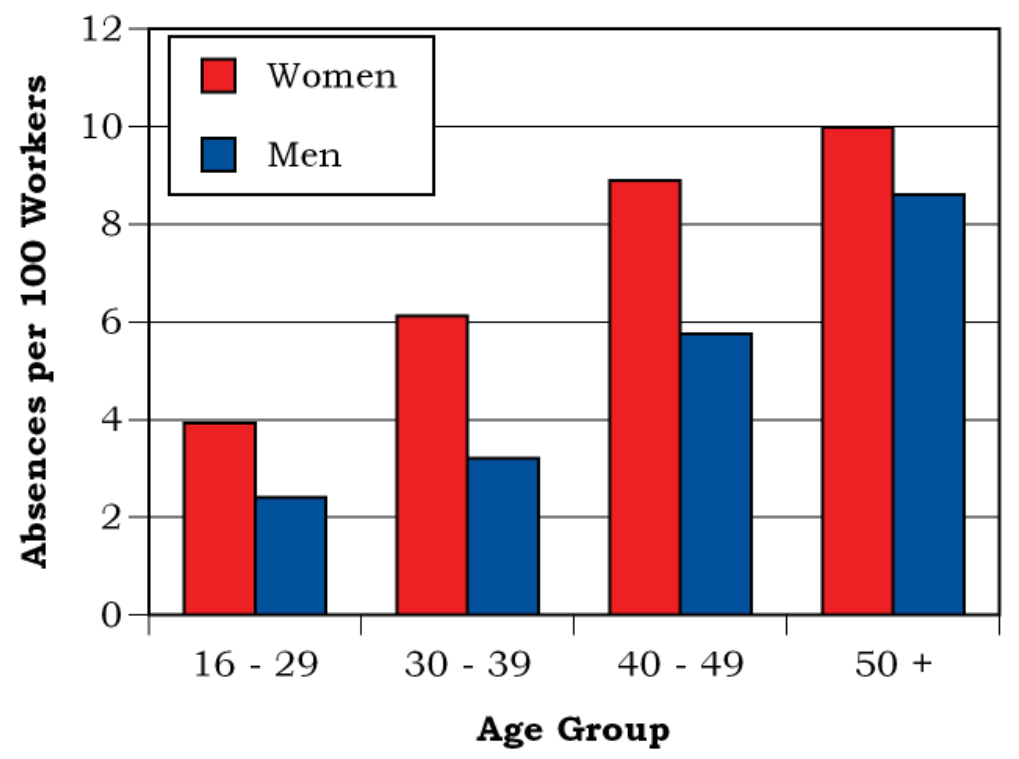

Figure 4. Number of Days Absent by Gender and Age

\begin{tabular}{|c|c|c|c|c|}
\hline \multirow{3}{*}{ Gender } & \multirow{2}{*}{ Age } & Number of & \multicolumn{2}{|c|}{ Number of Days Absent } \\
\cline { 3 - 5 } & & Absences & Total & Average \\
\hline \multirow{4}{*}{ Women } & $16-29$ & 13 & 262 & 20 \\
\cline { 2 - 5 } & $30-39$ & 29 & 896 & 31 \\
\cline { 2 - 5 } & $40-49$ & 83 & 2,464 & 30 \\
\cline { 2 - 5 } & $50+$ & 108 & 2,920 & 27 \\
\cline { 2 - 5 } & Total & 233 & 6,542 & 28 \\
\hline \multirow{4}{*}{ Men } & $16-29$ & 14 & 344 & 25 \\
\cline { 2 - 5 } & $30-39$ & 32 & 536 & 17 \\
\cline { 2 - 5 } & $40-49$ & 101 & 2,270 & 22 \\
\cline { 2 - 5 } & $50+$ & 249 & 5,543 & 22 \\
\cline { 2 - 5 } & Total & 396 & 8,693 & 22 \\
\hline
\end{tabular}


Figure 5. Absence Rate by Job Category and Gender

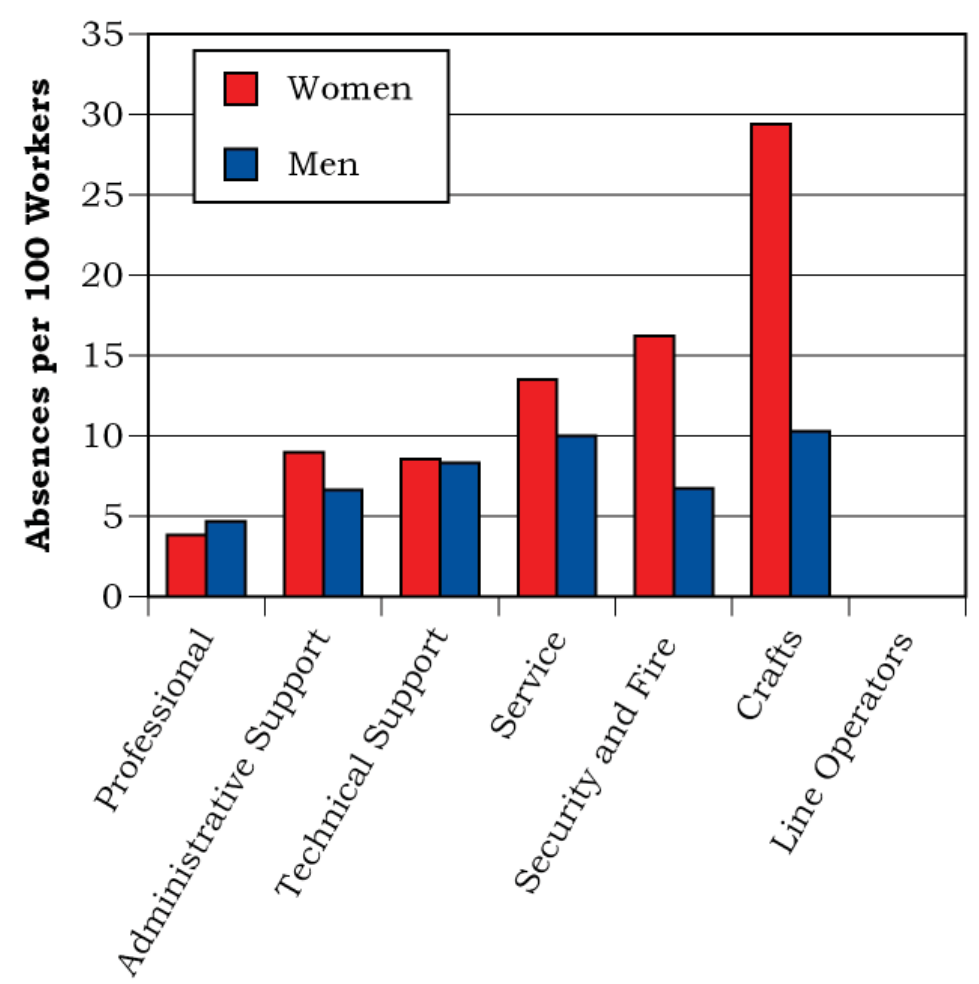

Job Category

Figure 6. Average Duration of Absence by Job Category and Gender

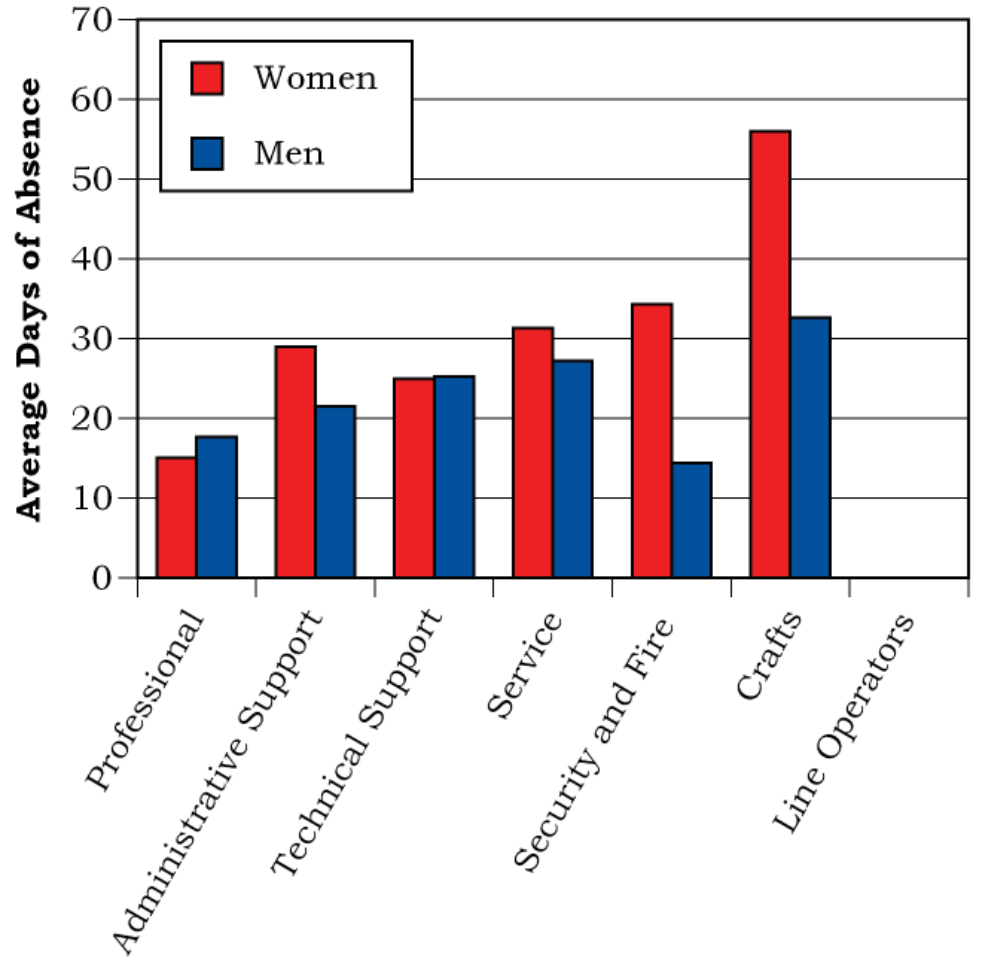




\section{Diagnostic Categories}

\section{Figure 7. Number of Diagnoses and Lost Calendar Days by Diagnostic Category (Categorized by ICD-9-CM) and Gender}

\begin{tabular}{|c|c|c|c|c|}
\hline \multirow[b]{2}{*}{ Diagnostic Category } & \multicolumn{2}{|c|}{ Women } & \multicolumn{2}{|c|}{ Men } \\
\hline & $\begin{array}{l}\text { Number of } \\
\text { Diagnoses }\end{array}$ & $\begin{array}{l}\text { Number } \\
\text { of Lost } \\
\text { Calendar } \\
\text { Days }\end{array}$ & $\begin{array}{l}\text { Number of } \\
\text { Diagnoses }\end{array}$ & $\begin{array}{c}\text { Number } \\
\text { of Lost } \\
\text { Calendar } \\
\text { Days }\end{array}$ \\
\hline Benign Growths & 10 & 503 & 8 & 240 \\
\hline Blood & 0 & 0 & 0 & 0 \\
\hline Cancer & 5 & 237 & 15 & 844 \\
\hline Digestive & 30 & 722 & 47 & 764 \\
\hline Endocrine/Metabolic & 17 & 516 & 10 & 154 \\
\hline Existing Birth Condition & 2 & 38 & 1 & 1 \\
\hline Genitourinary & 42 & 935 & 15 & 142 \\
\hline Heart/Circulatory & 9 & 152 & 31 & 871 \\
\hline Infections / Parasites & 13 & 314 & 20 & 253 \\
\hline Injury & 21 & 684 & 88 & 1,897 \\
\hline Miscarriage & 1 & 5 & NA & NA \\
\hline Musculoskeletal & 40 & 1,805 & 84 & 2,151 \\
\hline Nervous System & 15 & 261 & 28 & 674 \\
\hline Psychological & 7 & 494 & 4 & 135 \\
\hline Respiratory & 47 & 423 & 69 & 651 \\
\hline Skin & 13 & 359 & 11 & 173 \\
\hline Unspecified Symptoms & 10 & 106 & 25 & 438 \\
\hline
\end{tabular}

Note: Lost calendar days for each absence are counted more than once when multiple diagnoses occur in different diagnostic categories for the same absence. 
Figure 8. Common Diagnoses Among Female Workers in 2006

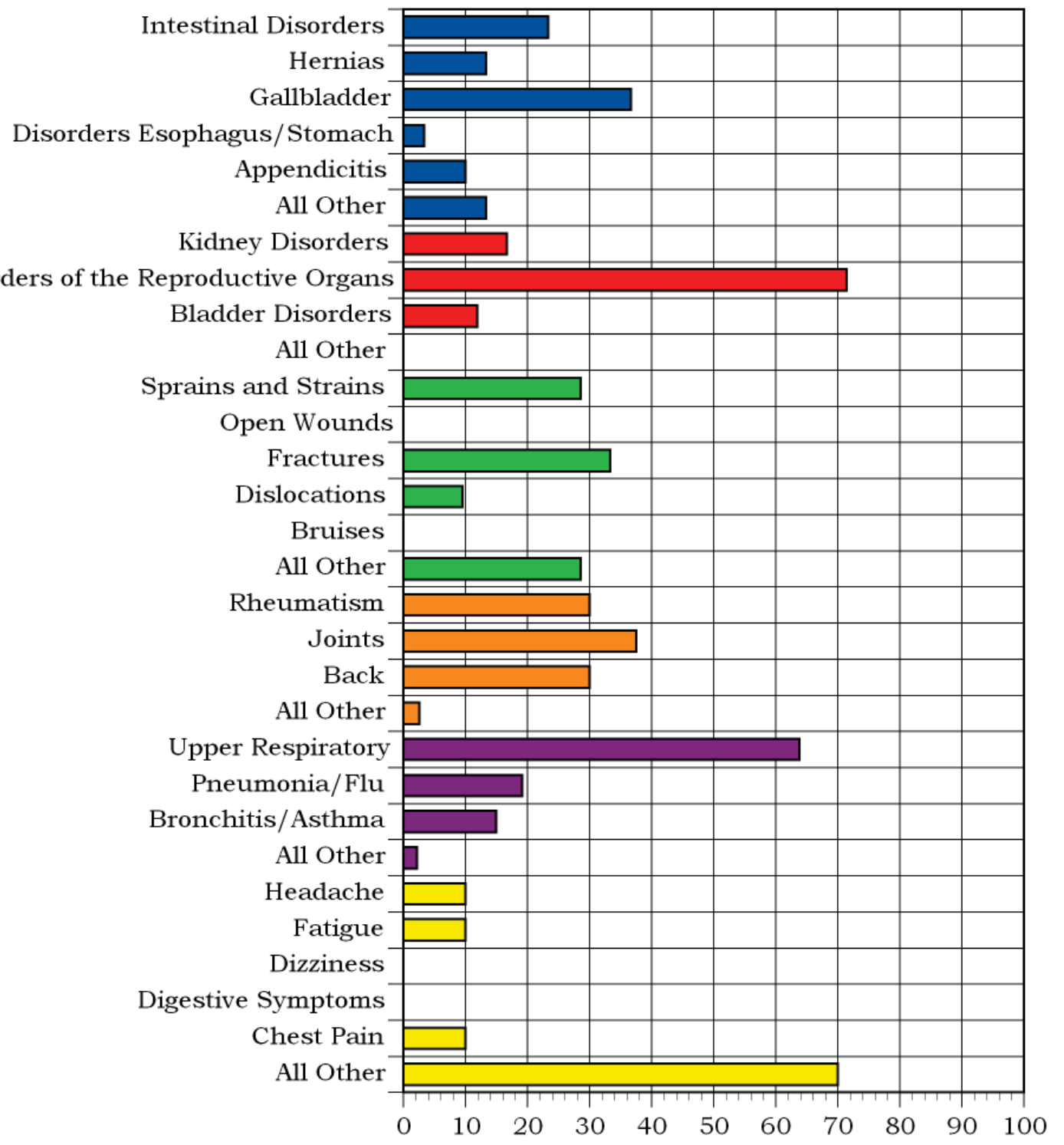

Percent Distribution of Diagnoses Within Diagnostic Category

Digestive, 30 Diagnoses

Genitourinary, 42 Diagnoses

Injury, 21 Diagnoses
Musculoskeletal, 40 Diagnoses

Respiratory, 47 Diagnoses

Unspecified Symptoms, 10 Diagnoses 
Figure 9. Common Diagnoses Among Male Workers in 2006

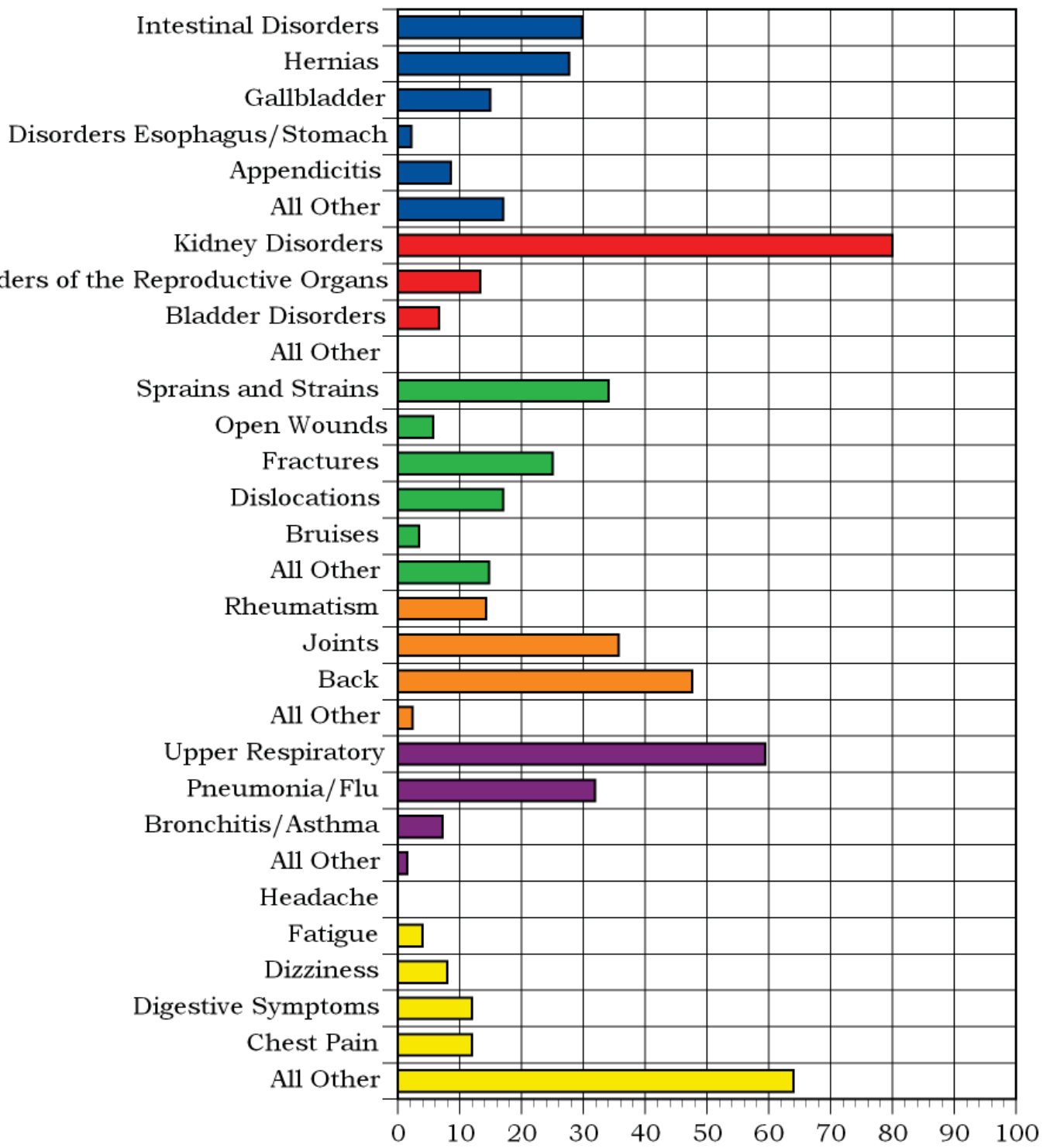

Percent Distribution of Diagnoses Within Diagnostic Category

\begin{tabular}{ll}
$\square$ Digestive, 47 Diagnoses & $\square$ Musculoskeletal, 84 Diagnoses \\
$\square$ Genitourinary, 15 Diagnoses & $\square$ Respiratory, 69 Diagnoses \\
$\square$ Injury, 88 Diagnoses & $\square$ Unspecified Symptoms, 25 Diagnoses \\
\hline
\end{tabular}




\section{Figure 10. Number of Most Frequently Reported Diagnoses by Job Category and Gender}

\begin{tabular}{|c|c|c|c|c|}
\hline Job Category & \multicolumn{2}{|l|}{ Men } & \multicolumn{2}{|l|}{ Women } \\
\hline \multirow{5}{*}{ Professional } & Injury & 24 & Respiratory & 5 \\
\hline & Respiratory & 24 & Cancer & 3 \\
\hline & Digestive & 21 & Digestive & 3 \\
\hline & & & Genitourinary & 3 \\
\hline & & & Skin & 3 \\
\hline \multirow{3}{*}{ Administrative Support } & Musculoskeletal & 34 & Genitourinary & 33 \\
\hline & Injury & 32 & Respiratory & 28 \\
\hline & Respiratory & 23 & Musculoskeletal & 26 \\
\hline \multirow{3}{*}{ Technical Support } & Musculoskeletal & 22 & Musculoskeletal & 8 \\
\hline & Injury & 12 & Digestive & 4 \\
\hline & Respiratory & 12 & Respiratory & 4 \\
\hline \multirow{5}{*}{ Service } & Musculoskeletal & 7 & Respiratory & 7 \\
\hline & Unspecified Symptoms & 4 & Genitourinary & 2 \\
\hline & Cancer & 3 & Musculoskeletal & 2 \\
\hline & Injury & 3 & & \\
\hline & Respiratory & 3 & & \\
\hline \multirow{6}{*}{ Security and Fire } & Injury & 7 & Genitourinary & 2 \\
\hline & Respiratory & 5 & Infections/Parasites & 1 \\
\hline & Unspecified Symptoms & 4 & Injury & 1 \\
\hline & & & Musculoskeletal & 1 \\
\hline & & & Respiratory & 1 \\
\hline & & & Unspecified Symptoms & 1 \\
\hline \multirow{5}{*}{ Crafts } & Injury & 10 & Benign Growths & 2 \\
\hline & Musculoskeletal & 6 & Respiratory & 2 \\
\hline & Digestive & 4 & Existing Birth Condition & 1 \\
\hline & Heart/Circulatory & 4 & Musculoskeletal & 1 \\
\hline & & & Nervous System & 1 \\
\hline Line Operators & & 0 & & $\mathbf{0}$ \\
\hline
\end{tabular}




\section{Rates of Disease Occurrence}

Figure 11. Rates for All Illnesses and Injuries Combined by Job Category, Gender, and Age

\begin{tabular}{|c|c|c|c|c|}
\hline \multirow{2}{*}{$\begin{array}{c}\text { All Illnesses \& } \\
\text { Injuries Combined }\end{array}$} & \multicolumn{4}{|c|}{ Rate per 1,000} \\
\hline & Job Category & Age & Men & Women \\
\hline & \multirow{2}{*}{ Professional } & $<50$ & 31 & 42 \\
\hline & & $50+$ & 73 & 47 \\
\hline & \multirow{2}{*}{ Administrative Support } & $<50$ & 55 & 86 \\
\hline & & $50+$ & 110 & 148 \\
\hline & \multirow{2}{*}{ Technical Support } & $<50$ & 64 & 95 \\
\hline & & $50+$ & 149 & 96 \\
\hline & \multirow{2}{*}{ Service } & $<50$ & 163 & 143 \\
\hline & & $50+$ & 86 & 170 \\
\hline & \multirow{2}{*}{ Security and Fire } & $<50$ & 60 & 103 \\
\hline & & $50+$ & 103 & 500 \\
\hline & \multirow{2}{*}{ Crafts } & $<50$ & 79 & 500 \\
\hline & & $50+$ & 134 & 333 \\
\hline & \multirow{2}{*}{ Line Operators } & $<50$ & 0 & 0 \\
\hline & & $50+$ & 0 & 0 \\
\hline
\end{tabular}

Figure 12. Rates for Selected Diagnostic Categories by Job Category, Gender, and Age

\begin{tabular}{|c|c|c|c|c|}
\hline \multirow{2}{*}{ Cancer } & \multicolumn{4}{|c|}{ Rate per 1,000} \\
\hline & Job Category & Age & Men & Women \\
\hline & Dugfonainas 1 & $<50$ & 1 & 0 \\
\hline & Prolessionad & $50+$ & 6 & 16 \\
\hline & Adminintrotime Gunnout & $<50$ & 0 & 1 \\
\hline & Administrative support & $50+$ & 2 & 0 \\
\hline & Technical Sunnort & $<50$ & 0 & 5 \\
\hline & I ecninical support & $50+$ & 0 & 0 \\
\hline & Service & $<50$ & 19 & 0 \\
\hline & Service & $50+$ & 9 & 0 \\
\hline & Security and Fire & $<50$ & 0 & 0 \\
\hline & Security and Fire & $50+$ & 0 & 0 \\
\hline & Crafts & $<50$ & 0 & 0 \\
\hline & Cratts & $50+$ & 6 & 0 \\
\hline & I ine Oneretors & $<50$ & 0 & 0 \\
\hline & Line uperators & $50+$ & 0 & 0 \\
\hline
\end{tabular}




\section{Figure 12. Rates for Selected Diagnostic Categories by Job Category, Gender, and Age (Continued)}

\begin{tabular}{|c|c|c|c|c|}
\hline \multirow{2}{*}{ Heart/Circulatory } & \multicolumn{4}{|c|}{ Rate per 1,000} \\
\hline & Job Category & Age & Men & Women \\
\hline & \multirow{2}{*}{ Professional } & $<50$ & 1 & 0 \\
\hline & & $50+$ & 8 & 0 \\
\hline & \multirow{2}{*}{ Administrative Support } & $<50$ & 0 & 3 \\
\hline & & $50+$ & 8 & 7 \\
\hline & \multirow{2}{*}{ Technical Support } & $<50$ & 10 & 5 \\
\hline & & $50+$ & 8 & 0 \\
\hline & \multirow{2}{*}{ Service } & $<50$ & 0 & 0 \\
\hline & & $50+$ & 9 & 0 \\
\hline & \multirow{2}{*}{ Security and Fire } & $<50$ & 0 & 0 \\
\hline & & $50+$ & 0 & 0 \\
\hline & \multirow{2}{*}{ Crafts } & $<50$ & 5 & 0 \\
\hline & & $50+$ & 17 & 0 \\
\hline & \multirow{2}{*}{ Line Operators } & $<50$ & 0 & 0 \\
\hline & & $50+$ & 0 & 0 \\
\hline
\end{tabular}

\begin{tabular}{|c|c|c|c|c|}
\hline \multirow{2}{*}{ Respiratory } & \multicolumn{4}{|c|}{ Rate per 1,000} \\
\hline & Job Category & Age & Men & Women \\
\hline & \multirow{2}{*}{ Professional } & $<50$ & 5 & 10 \\
\hline & & $50+$ & 13 & 5 \\
\hline & \multirow{2}{*}{ Administrative Support } & $<50$ & 9 & 10 \\
\hline & & $50+$ & 17 & 24 \\
\hline & \multirow{2}{*}{ Technical Support } & $<50$ & 8 & 9 \\
\hline & & $50+$ & 22 & 19 \\
\hline & \multirow{2}{*}{ Service } & $<50$ & 19 & 61 \\
\hline & & $50+$ & 9 & 85 \\
\hline & \multirow{2}{*}{ Security and Fire } & $<50$ & 15 & 34 \\
\hline & & $50+$ & 17 & 0 \\
\hline & \multirow{2}{*}{ Crafts } & $<50$ & 5 & 250 \\
\hline & & $50+$ & 6 & 0 \\
\hline & \multirow{2}{*}{ Line Operators } & $<50$ & 0 & 0 \\
\hline & & $50+$ & 0 & 0 \\
\hline
\end{tabular}


Figure 12. Rates for Selected Diagnostic Categories by Job Category, Gender, and Age (Continued)

\begin{tabular}{|c|c|c|c|c|}
\hline \multirow{2}{*}{ Injury } & \multicolumn{4}{|c|}{ Rate per 1,000} \\
\hline & Job Category & Age & Men & Women \\
\hline & Profecaionol 1 & $<50$ & 8 & 5 \\
\hline & Protessional & $50+$ & 10 & 0 \\
\hline & Aminiatrotim $\mathrm{G}$ unnont & $<50$ & 9 & 8 \\
\hline & Administrative Support & $50+$ & 27 & 10 \\
\hline & Tochninol Sunnort & $<50$ & 17 & 5 \\
\hline & I echnical support & $50+$ & 11 & 10 \\
\hline & Comrine & $<50$ & 0 & 20 \\
\hline & Service & $50+$ & 26 & 0 \\
\hline & Security and Fire & $<50$ & 15 & 1 \\
\hline & security and Fire & $50+$ & 52 & 125 \\
\hline & Crofta $\mathrm{C} \longrightarrow \mathrm{C}$ & $<50$ & 32 & 0 \\
\hline & Craits & $50+$ & 22 & 0 \\
\hline & I ine Onorotora $\mathrm{C}$ & $<50$ & 0 & 0 \\
\hline & Line uperators & $50+$ & 0 & 0 \\
\hline
\end{tabular}

Time Trends

Figure 13. Age-Adjusted Rates for All Diagnoses Combined Among Women and Men from 2003 to 2006*

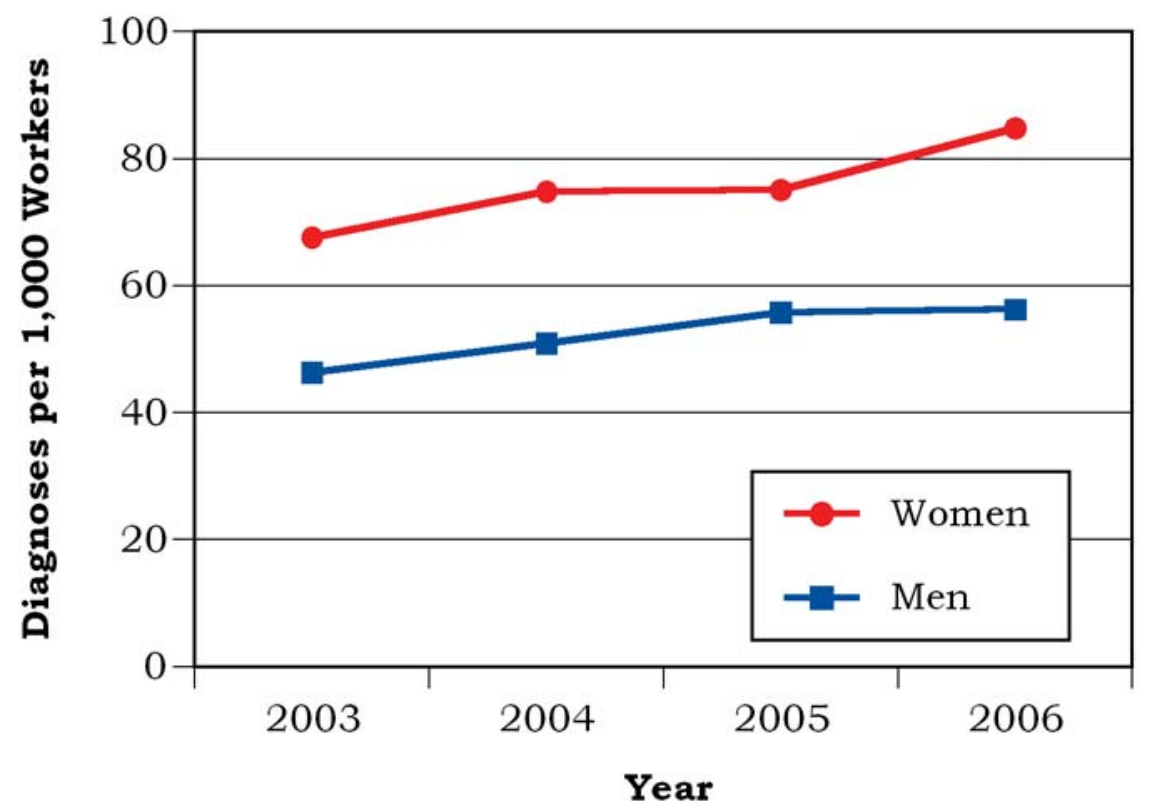

* Standardized to age distribution of 2000 U.S. population. 
Figure 14. Age-Adjusted Rates for Selected Diagnostic

Categories Among Women and Men from 2003 to 2006*

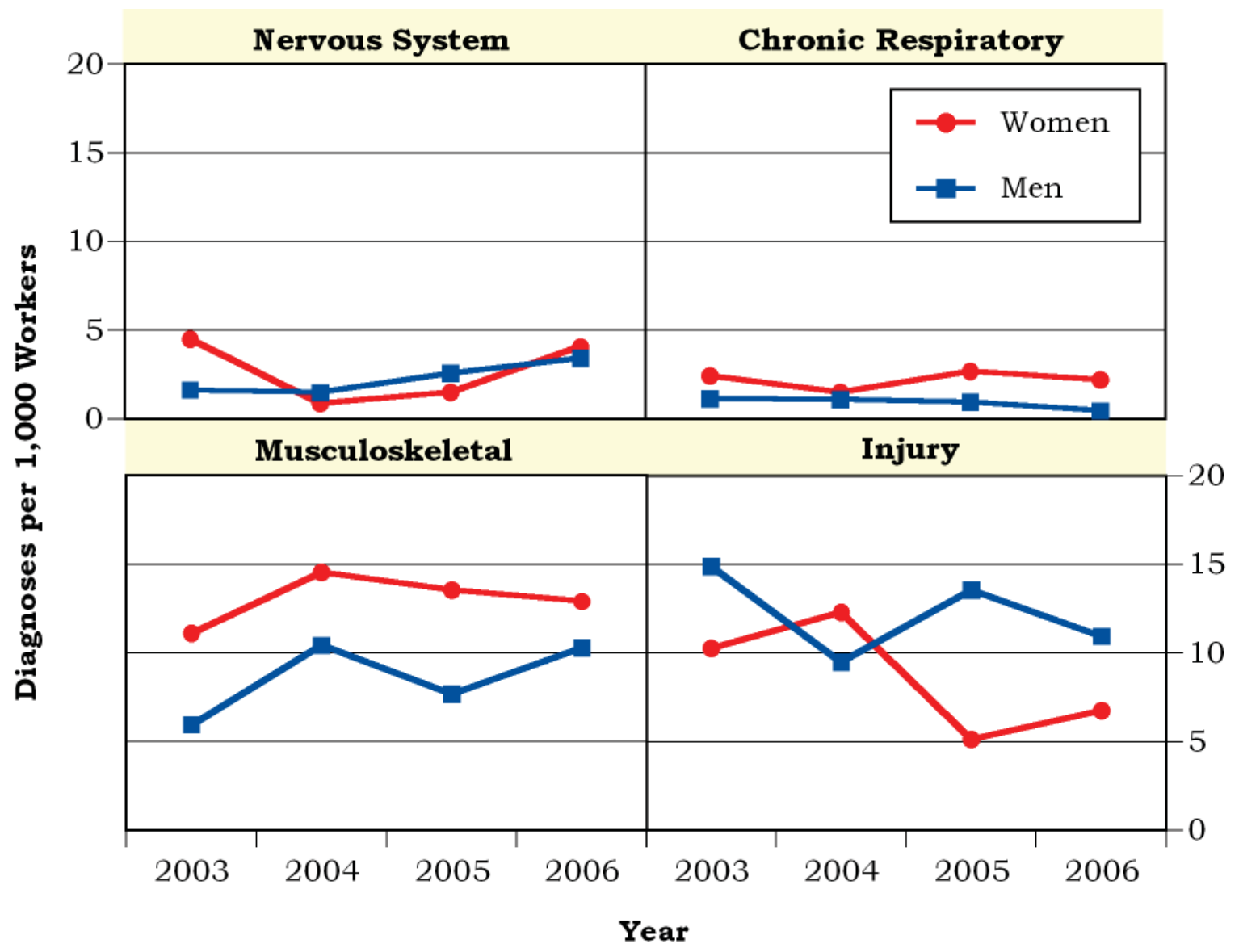

* Standardized to age distribution of 2000 U.S. population. 
Figure 15. Age-Adjusted Rates for All Diagnoses Combined Among Women and Men by Job Category from 2003 to 2006*
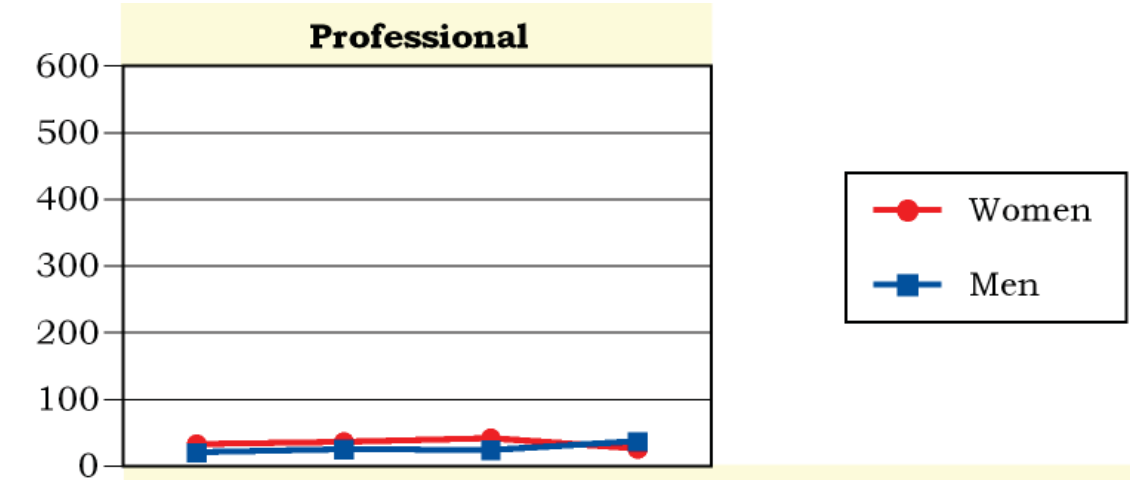

\section{Technical Support}

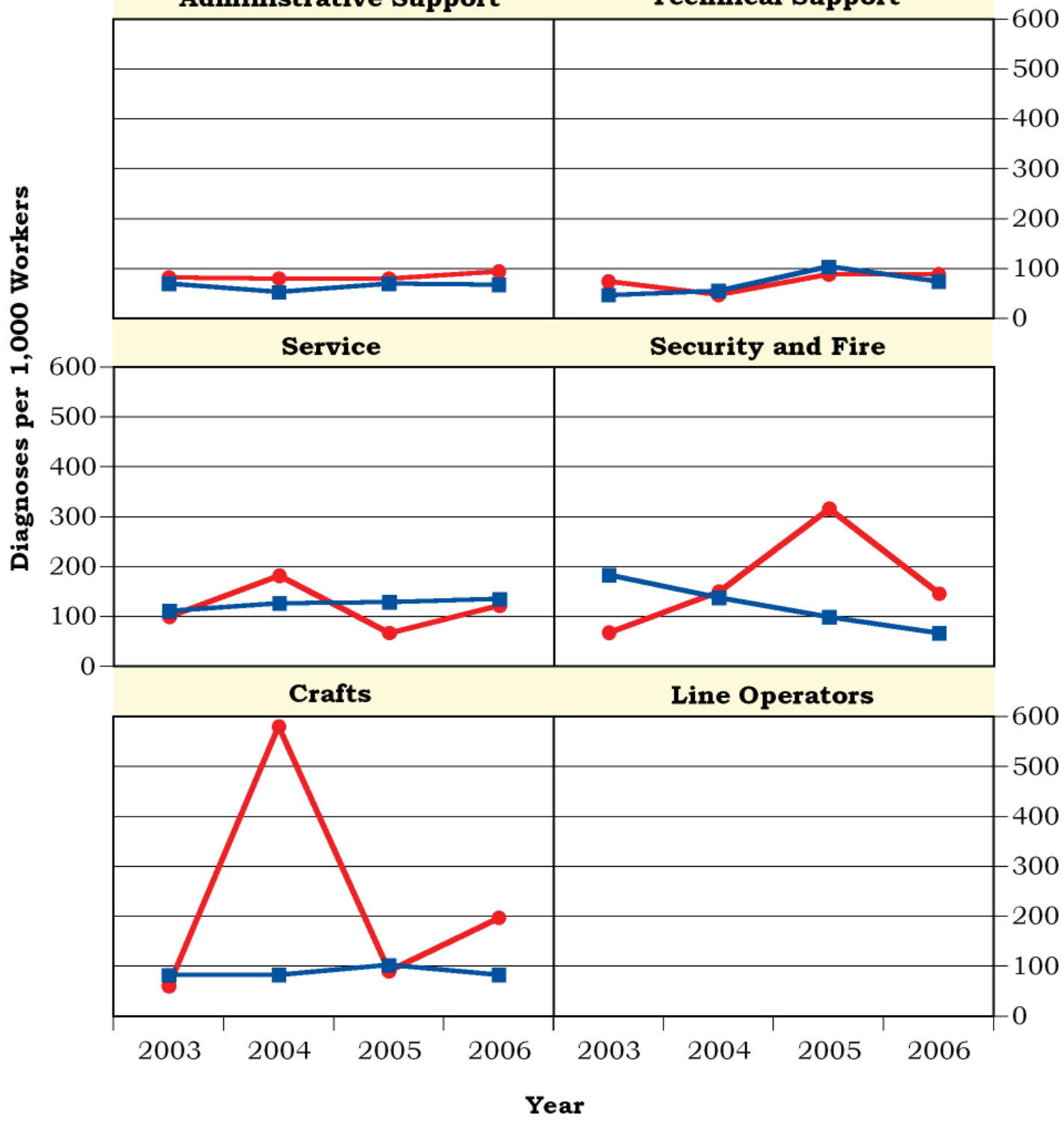

* Standardized to age distribution of 2000 U.S. population. 


\section{Sentinel Health Events for Occupations (SHEOs)}

An occupational sentinel health event (SHEO) is a disease, disability, or death that is likely to be occupationally related. Although sentinel health events may indicate an occupational exposure, many may result from nonoccupational exposures. Sentinel health events are therefore assessed in two categories:

Definite Sentinel Health Events: Diseases that are unlikely to occur in the absence of an occupational exposure (e.g., asbestosis).

Possible Sentinel Health Events: Diseases that may be occupational but can also occur in the absence of an occupational exposure (e.g., lung cancer or carpal tunnel syndrome).

Figure 16. Characteristics of SHEOs by Gender

\begin{tabular}{|l|c|c|c|c|}
\hline \multirow{2}{*}{} & \multicolumn{2}{|c|}{$\begin{array}{c}\text { Total Number of } \\
\text { SHEO Diagnoses }\end{array}$} & \multicolumn{2}{c|}{$\begin{array}{c}\text { Total Number of } \\
\text { Days Absent }\end{array}$} \\
\cline { 2 - 5 } & Men & Women & Men & Women \\
\hline Definite & 0 & 0 & 0 & 0 \\
\hline Possible & 6 & 2 & 236 & 85 \\
\hline Total & 6 & 2 & 236 & 85 \\
\hline
\end{tabular}

Figure 17. SHEO Diagnoses by Gender

\begin{tabular}{|l|c|c|}
\hline \multirow{2}{*}{\multicolumn{1}{|c|}{ Diagnoses }} & \multicolumn{2}{c|}{ Gender } \\
\cline { 2 - 3 } & Women & Men \\
\hline Carpel Tunnel Syndrome & 2 & 3 \\
\hline Pneumonconiosis (Berylliosis) & $\mathbf{0}$ & $\mathbf{0}$ \\
\hline Musculoskeletal Conditions & $\mathbf{0}$ & $\mathbf{0}$ \\
\hline Injuries & $\mathbf{0}$ & $\mathbf{0}$ \\
\hline Other Conditions & $\mathbf{0}$ & $\mathbf{3}$ \\
\hline
\end{tabular}


Occupational Safety and Health Administration (OSHA)-Recordable Events

Figure 18. OSHA-Recordable Events by Gender and Age

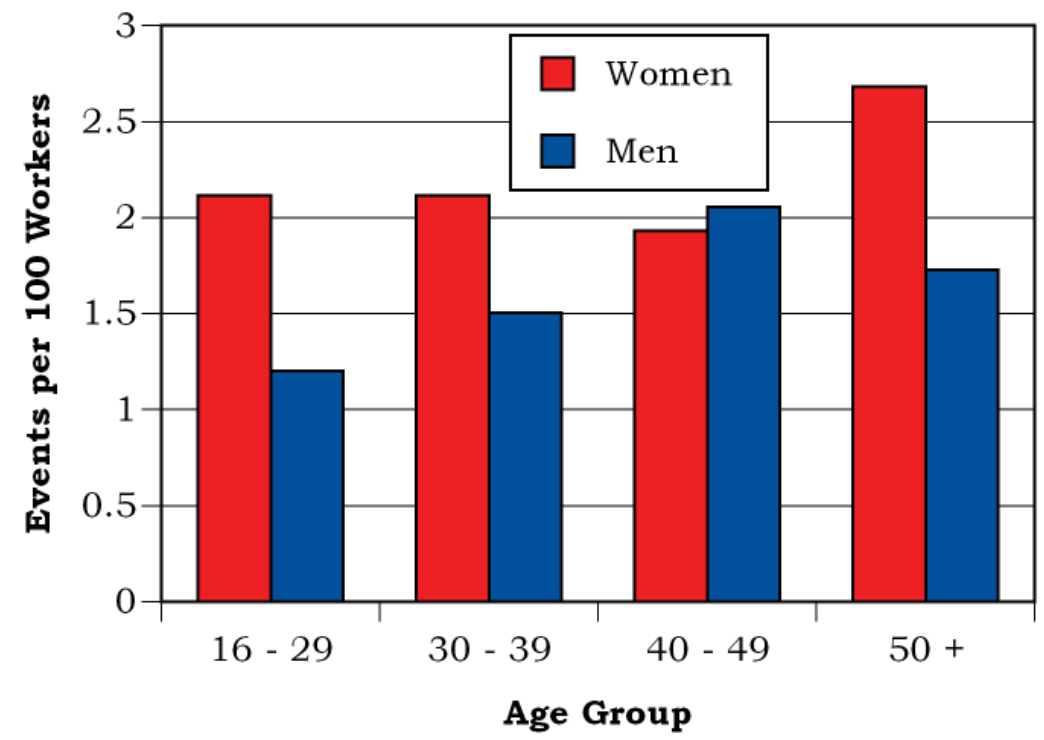

Figure 19. OSHA-Recordable Events by Job Category and Gender

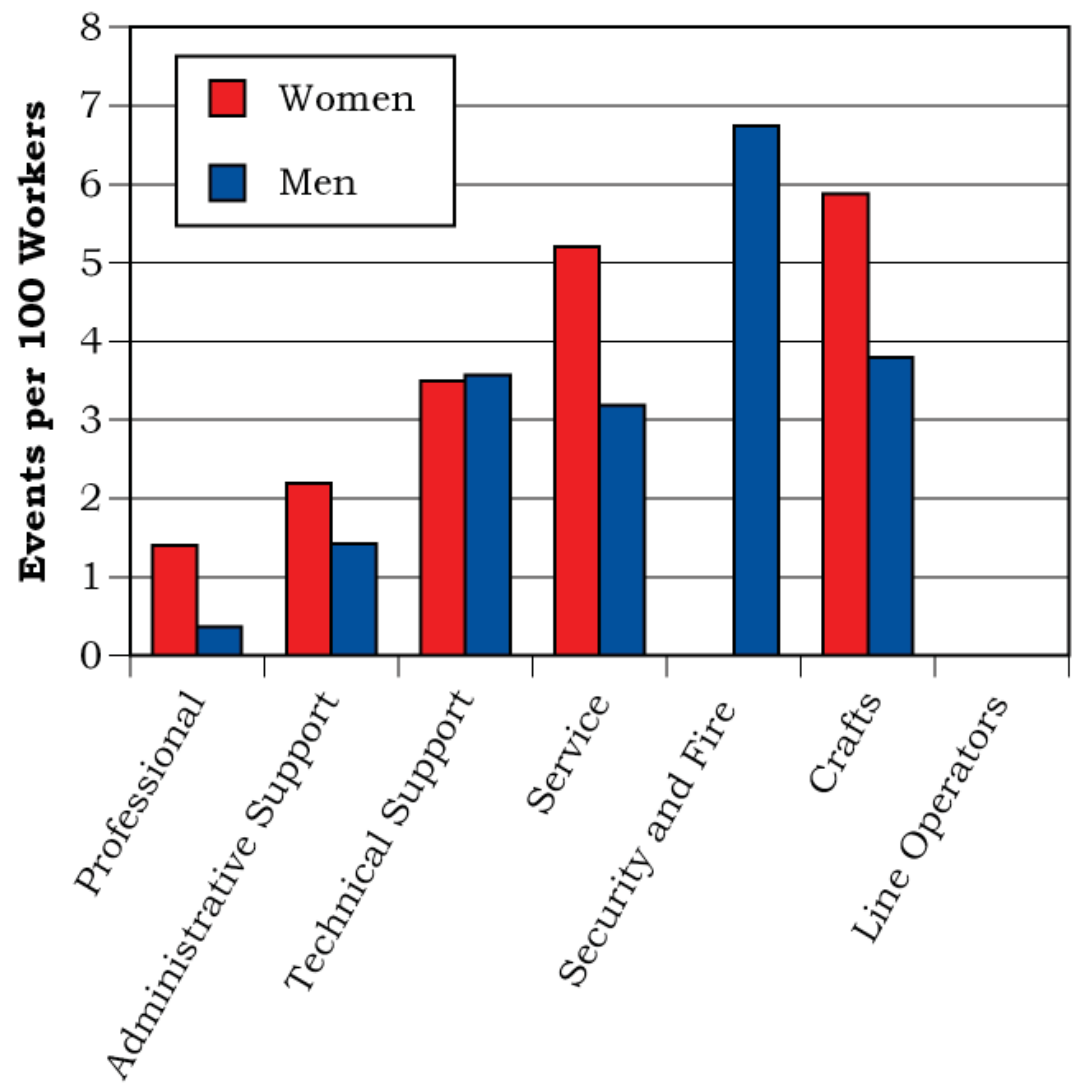

Job Category 


\section{Diagnostic and Accident Categories for OSHA-Recordable Events}

Figure 20. OSHA-Recordable Diagnoses by Diagnostic Category and Gender

\begin{tabular}{|l|c|c|}
\hline \multirow{2}{*}{ Diagnostic Category } & \multicolumn{2}{|c|}{ Gender } \\
\cline { 2 - 3 } & Women & Men \\
\hline Infections / Parasites & $\mathbf{1}$ & $\mathbf{2}$ \\
\hline Musculoskeletal & $\mathbf{6 2}$ & $\mathbf{5 6}$ \\
\hline Nervous System & $\mathbf{0}$ & $\mathbf{3}$ \\
\hline Respiratory & $\mathbf{1}$ & $\mathbf{3}$ \\
\hline Skin & $\mathbf{1 2}$ & $\mathbf{1 3}$ \\
\hline Unspecified Symptoms & $\mathbf{4 4}$ & $\mathbf{1 0 4}$ \\
\hline Injury & $\mathbf{0}$ & $\mathbf{1}$ \\
\hline Fractures - Neck, Trunk & $\mathbf{1}$ & $\mathbf{9}$ \\
\hline Fractures - Upper Limb & $\mathbf{1}$ & $\mathbf{1}$ \\
\hline Fractures - Lower Limb & $\mathbf{7}$ & $\mathbf{1 4}$ \\
\hline Back Sprains \& Strains & $\mathbf{6}$ & $\mathbf{3 3}$ \\
\hline Other Sprains \& Strains & $\mathbf{1}$ & $\mathbf{2}$ \\
\hline Intracranial Injuries & $\mathbf{1}$ & $\mathbf{2}$ \\
\hline Open Wounds - Head, Neck, Trunk & $\mathbf{0}$ & $\mathbf{1 1}$ \\
\hline Open Wounds - Upper Limb & $\mathbf{0}$ & $\mathbf{1}$ \\
\hline Open Wounds - Lower Limb & $\mathbf{7}$ & $\mathbf{2}$ \\
\hline Superficial Injuries & $\mathbf{4}$ & $\mathbf{9}$ \\
\hline Bruises & $\mathbf{1}$ & $\mathbf{2}$ \\
\hline Foreign Bodies Entering Orifice & $\mathbf{0}$ & $\mathbf{2}$ \\
\hline Burns & $\mathbf{0}$ & $\mathbf{1}$ \\
\hline Injuries to Nerves \& Spinal Cord & $\mathbf{0}$ & $\mathbf{1 3}$ \\
\hline Unspecified Injuries & & \\
\hline Adverse Reactions to Non-Medical & & \\
\hline Substances & & \\
\hline
\end{tabular}


Figure 21. OSHA-Recordable Accidents by Type and Gender

\begin{tabular}{|l|c|c|}
\hline \multirow{2}{*}{ Accident Category } & \multicolumn{2}{|c|}{ Gender } \\
\cline { 2 - 3 } & Women & Men \\
\cline { 2 - 3 } & $\begin{array}{c}\text { Number of } \\
\text { Accidents }\end{array}$ & $\begin{array}{c}\text { Number of } \\
\text { Accidents }\end{array}$ \\
\hline Motor Vehicle Traffic & $\mathbf{2}$ & $\mathbf{1}$ \\
\hline Non-Motor Vehicle & $\mathbf{1}$ & $\mathbf{4}$ \\
\hline Falls & $\mathbf{1 1}$ & $\mathbf{1 1}$ \\
\hline Natural/Environmental Factors & $\mathbf{0}$ & $\mathbf{3}$ \\
\hline Submersion/Suffocation/Foreign Bodies & $\mathbf{1}$ & $\mathbf{2}$ \\
\hline Drug Reaction & $\mathbf{0}$ & $\mathbf{1}$ \\
\hline Other Accidents & $\mathbf{4 7}$ & $\mathbf{7 6}$ \\
\hline Struck by an Object & $\mathbf{2}$ & $\mathbf{5}$ \\
\hline Caught Between Objects & $\mathbf{0}$ & $\mathbf{2}$ \\
\hline Cutting/Piercing Instrument/Object & $\mathbf{0}$ & $\mathbf{7}$ \\
\hline Firearm & $\mathbf{0}$ & $\mathbf{1}$ \\
\hline Hot, Corrosive, or Caustic Material/ & $\mathbf{0}$ & $\mathbf{2}$ \\
\hline Steam & & $\mathbf{4 1}$ \\
\hline Overexertion/Strenuous Movements & $\mathbf{1 3}$ & $\mathbf{1}$ \\
\hline Noise & $\mathbf{0}$ & $\mathbf{1 7}$ \\
\hline Repetitive Trauma & $\mathbf{3 2}$ & $\mathbf{9 8}$ \\
\hline Total & $\mathbf{6 2}$ & \\
\hline
\end{tabular}




\section{Rates of OSHA-Recordable Events}

Figure 22. OSHA-Recordable Rates by Age and Job Categories Among Women, All Diagnoses Combined

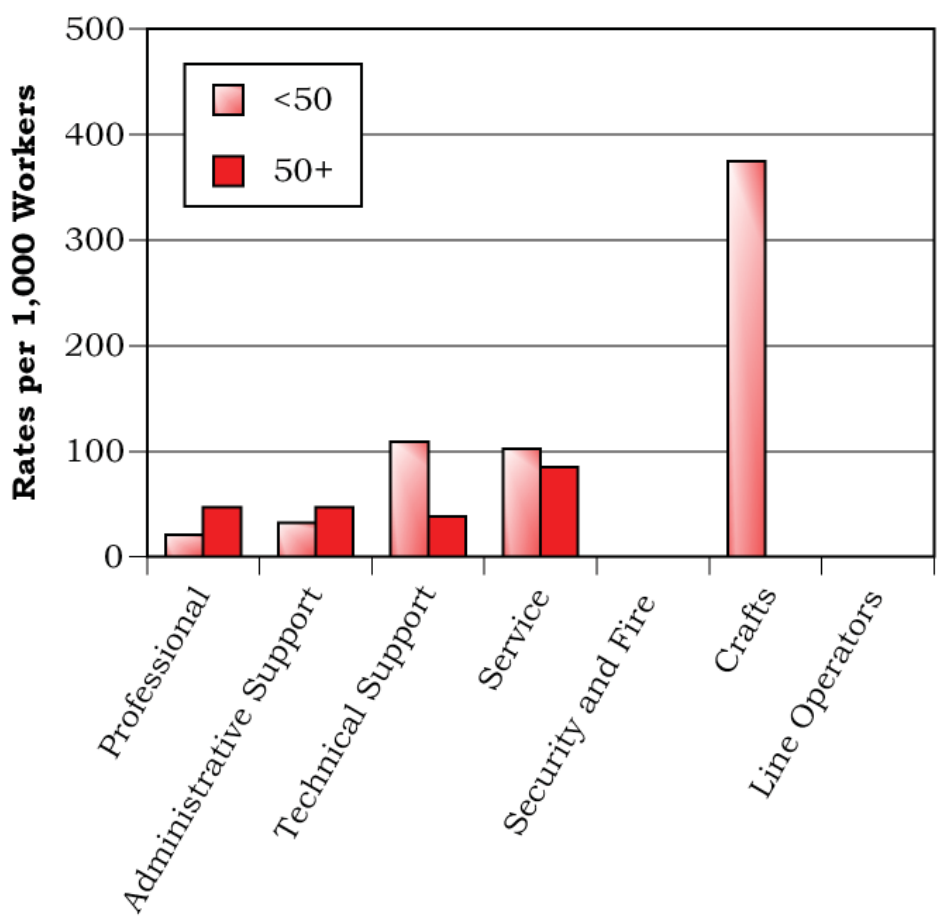

Job Category

Figure 23. OSHA-Recordable Rates by Age and Job Categories Among Men, All Diagnoses Combined

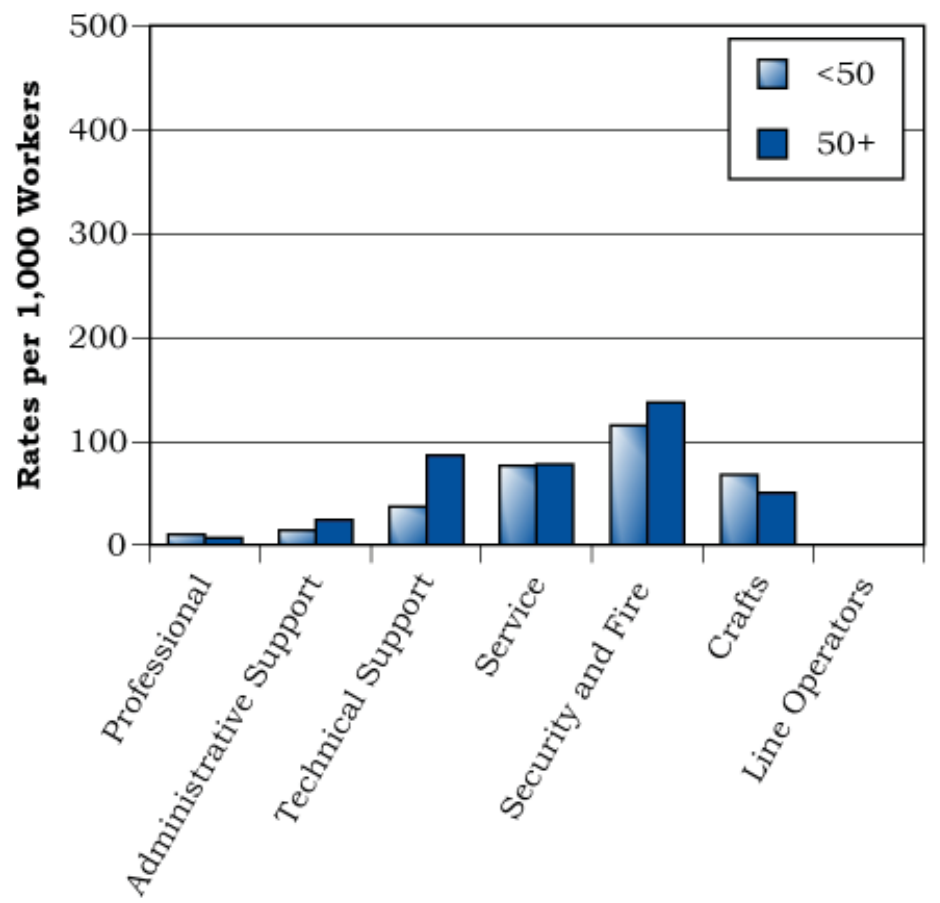

Job Category 


\section{Time Trends for OSHA-Recordable Events}

Figure 24. Age-Adjusted Rates for All OSHA-Recordable Diagnoses Combined Among Women and Men by Job Category from 2003 to 2006*

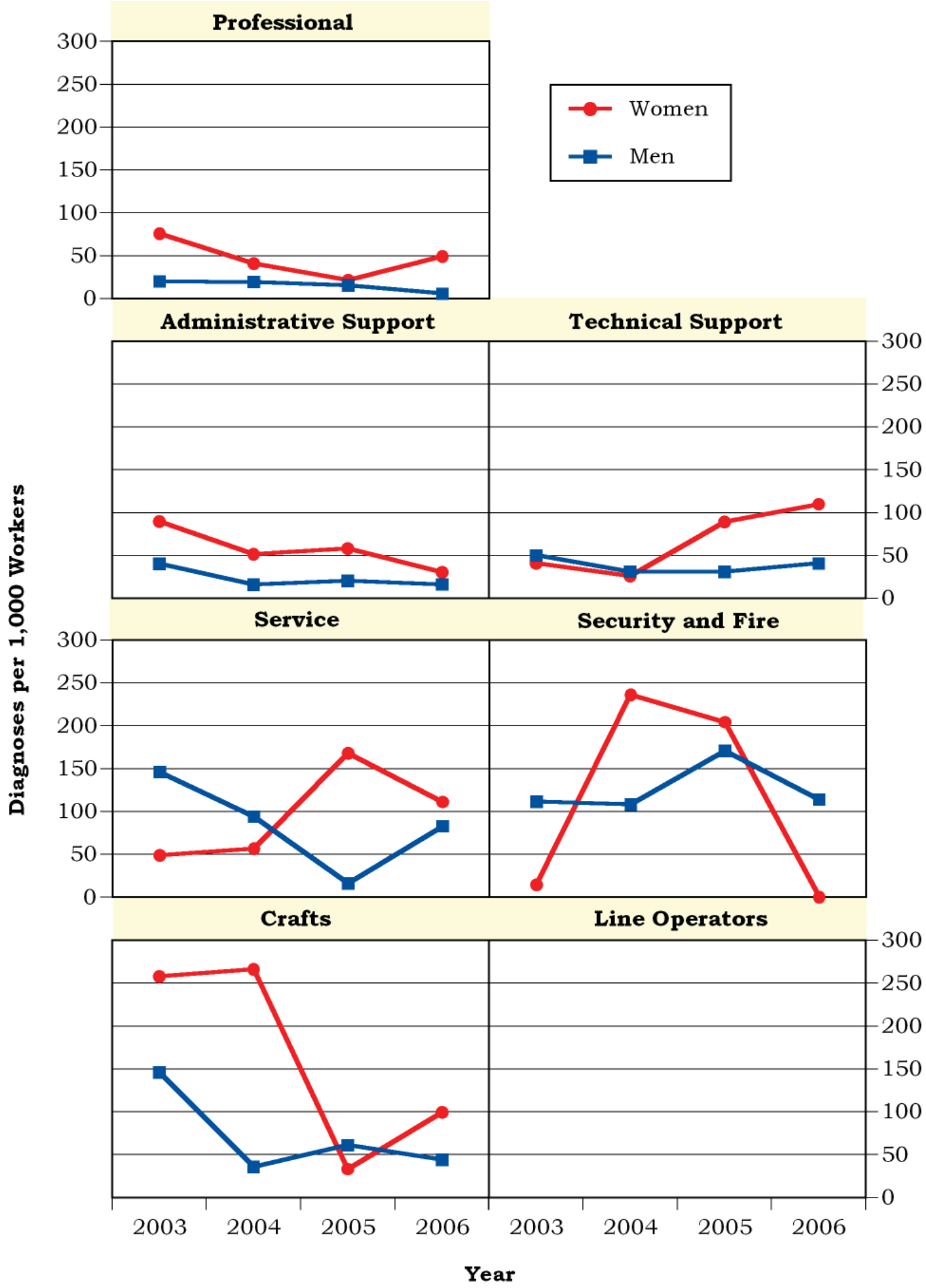

* Standardized to age distribution of 2000 U.S. population. 


\section{Appendices}


Lawrence Livermore National Laboratory 2006

Absence Data

Appendix A. Work Force by Gender, Age, and Job Category

\begin{tabular}{|c|c|c|c|c|c|c|c|c|c|c|c|}
\hline \multirow{3}{*}{ Job Category } & \multicolumn{5}{|c|}{ Women } & \multicolumn{5}{|c|}{ Men } & \multirow[b]{3}{*}{ TOTAL } \\
\hline & \multicolumn{4}{|c|}{ Age Group } & \multirow[b]{2}{*}{ TOTAL } & \multicolumn{4}{|c|}{ Age Group } & \multirow[b]{2}{*}{ TOTAL } & \\
\hline & $16-29$ & 30 - 39 & $40-49$ & $50+$ & & $16-29$ & 30 - 39 & $40-49$ & $50+$ & & \\
\hline Professional & 35 & 123 & 223 & 190 & 571 & 117 & 466 & 816 & 1,311 & 2,710 & 3,281 \\
\hline Administrative Support & 228 & 259 & 571 & 723 & 1,781 & 334 & 186 & 368 & 874 & 1,762 & 3,543 \\
\hline Technical Support & 56 & 70 & 85 & 104 & 315 & 46 & 155 & 283 & 356 & 840 & 1,155 \\
\hline Service & 7 & 9 & 33 & 47 & 96 & 13 & 35 & 56 & 116 & 220 & 316 \\
\hline Security and Fire & 5 & 11 & 13 & 8 & 37 & 60 & 114 & 94 & 58 & 326 & 363 \\
\hline Crafts & 0 & 1 & 7 & 9 & 17 & 13 & 42 & 135 & 179 & 369 & 386 \\
\hline TOTAL & 331 & 473 & 932 & 1,081 & 2,817 & 583 & 998 & 1,752 & 2,894 & 6,227 & 9,044 \\
\hline
\end{tabular}

Appendix B. Age Distribution of the Work Force by Gender

\begin{tabular}{|c|r|r|r|r|r|r|r|c|}
\hline \multirow{3}{*}{ Year } & \multicolumn{4}{|c|}{ Women } & \multicolumn{4}{c|}{ Men } \\
\cline { 2 - 9 } & \multicolumn{3}{|c|}{$\begin{array}{c}\text { Percent Distribution by Age } \\
\text { Group }\end{array}$} & \multicolumn{4}{|c|}{$\begin{array}{c}\text { Percent Distribution by Age } \\
\text { Group }\end{array}$} \\
\cline { 2 - 9 } & $\mathbf{1 6 - 2 9}$ & $\mathbf{3 0}-\mathbf{3 9}$ & $\mathbf{4 0}-\mathbf{4 9}$ & $\mathbf{5 0}+$ & $\mathbf{1 6}-\mathbf{2 9}$ & $\mathbf{3 0}-\mathbf{3 9}$ & $\mathbf{4 0}-\mathbf{4 9}$ & $\mathbf{5 0}+$ \\
\hline $\mathbf{2 0 0 2}$ & 13.04 & 19.68 & 35.45 & 31.83 & 10.25 & 18.23 & 32.16 & 39.35 \\
\hline $\mathbf{2 0 0 3}$ & 14.40 & 16.07 & 39.17 & 30.37 & 11.31 & 15.34 & 34.82 & 38.53 \\
\hline $\mathbf{2 0 0 4}$ & 14.34 & 16.24 & 37.93 & 31.48 & 11.59 & 14.87 & 33.85 & 39.70 \\
\hline $\mathbf{2 0 0 5}$ & 12.77 & 16.87 & 33.95 & 36.41 & 10.41 & 16.04 & 28.99 & 44.56 \\
\hline $\mathbf{2 0 0 6}$ & 11.75 & 16.79 & 33.08 & 38.37 & 9.36 & 16.03 & 28.14 & 46.48 \\
\hline
\end{tabular}


Lawrence Livermore National Laboratory 2006

Absence Data

Appendix C. Total Number of Workers Who Reported at Least One Absence by Gender, Age, and Job Category*

\begin{tabular}{|c|c|c|c|c|c|c|c|c|c|c|c|}
\hline \multirow{3}{*}{ Job Category } & \multicolumn{5}{|c|}{ Women } & \multicolumn{5}{|c|}{ Men } & \multirow[b]{3}{*}{ TOTAL } \\
\hline & \multicolumn{4}{|c|}{ Age Group } & \multirow[b]{2}{*}{ TOTAL } & \multicolumn{4}{|c|}{ Age Group } & \multirow[b]{2}{*}{ TOTAL } & \\
\hline & $16-29$ & 30 - 39 & $40-49$ & $50+$ & & $16-29$ & $30-39$ & $40-49$ & $50+$ & & \\
\hline Professional & 1 & 3 & 10 & 6 & 20 & 1 & 6 & 27 & 89 & 123 & 143 \\
\hline Administrative Support & 10 & 15 & 50 & 73 & 148 & 3 & 7 & 30 & 68 & 108 & 256 \\
\hline Technical Support & 1 & 6 & 10 & 7 & 24 & 1 & 7 & 17 & 39 & 64 & 88 \\
\hline Service & 1 & 0 & 5 & 7 & 13 & 2 & 1 & 9 & 7 & 19 & 32 \\
\hline Security and Fire & 0 & 2 & 1 & 1 & 4 & 6 & 9 & 1 & 6 & 22 & 26 \\
\hline Crafts & 0 & 0 & 3 & 2 & 5 & 1 & 1 & 13 & 21 & 36 & 41 \\
\hline TOTAL & 13 & 26 & 79 & 96 & 214 & 14 & 31 & 97 & 230 & 372 & 586 \\
\hline
\end{tabular}

*Only those job categories and gender/age combinations with at least one absence appear in this table.

Appendix D. Total Number of Absences by Gender, Age, and Job Category*

\begin{tabular}{|c|c|c|c|c|c|c|c|c|c|c|c|}
\hline \multirow{3}{*}{ Job Category } & \multicolumn{5}{|c|}{ Women } & \multicolumn{5}{|c|}{ Men } & \multirow[b]{3}{*}{ TOTAL } \\
\hline & \multicolumn{4}{|c|}{ Age Group } & \multirow[b]{2}{*}{ TOTAL } & \multicolumn{4}{|c|}{ Age Group } & \multirow[b]{2}{*}{ TOTAL } & \\
\hline & $16-29$ & 30 - 39 & $40-49$ & $50+$ & & $16-29$ & 30 - 39 & $40-49$ & $50+$ & & \\
\hline Professional & 1 & 3 & 10 & 8 & 22 & 1 & 6 & 28 & 92 & 127 & 149 \\
\hline Administrative Support & 10 & 17 & 53 & 80 & 160 & 3 & 8 & 30 & 76 & 117 & 277 \\
\hline Technical Support & 1 & 7 & 11 & 8 & 27 & 1 & 7 & 18 & 44 & 70 & 97 \\
\hline Service & 1 & 0 & 5 & 7 & 13 & 2 & 1 & 11 & 8 & 22 & 35 \\
\hline Security and Fire & 0 & 2 & 1 & 3 & 6 & 6 & 9 & 1 & 6 & 22 & 28 \\
\hline Crafts & 0 & 0 & 3 & 2 & 5 & 1 & 1 & 13 & 23 & 38 & 43 \\
\hline TOTAL & 13 & 29 & 83 & 108 & 233 & 14 & 32 & 101 & 249 & 396 & 629 \\
\hline
\end{tabular}

*Only those job categories and gender/age combinations with at least one absence appear in this table. 
Lawrence Livermore National Laboratory 2006

Absence Data

Appendix E. Distribution of the Number of Calendar Days Missed per Absence by Gender and Age*

\begin{tabular}{|c|c|c|c|c|c|c|c|c|c|c|c|}
\hline \multirow{3}{*}{$\begin{array}{c}\# \\
\text { of } \\
\text { Calendar } \\
\text { Days }\end{array}$} & \multicolumn{5}{|c|}{ Women } & \multicolumn{5}{|c|}{ Men } & \multirow[b]{3}{*}{ TOTAL } \\
\hline & \multicolumn{4}{|c|}{ Age Group } & \multirow[b]{2}{*}{ TOTAL } & \multicolumn{4}{|c|}{ Age Group } & \multirow[b]{2}{*}{ TOTAL } & \\
\hline & $16-29$ & 30 - 39 & $40-49$ & $50+$ & & 16 - 29 & $30-39$ & $40-49$ & $50+$ & & \\
\hline$<15$ & 10 & 12 & 48 & 56 & 126 & 9 & 24 & 65 & 152 & 250 & 376 \\
\hline $15-28$ & 2 & 10 & 9 & 23 & 44 & 2 & 3 & 17 & 48 & 70 & 114 \\
\hline $29-42$ & 0 & 1 & 6 & 8 & 15 & 0 & 1 & 5 & 14 & 20 & 35 \\
\hline $43-56$ & 0 & 1 & 6 & 4 & 11 & 1 & 2 & 5 & 13 & 21 & 32 \\
\hline $57-91$ & 0 & 1 & 9 & 12 & 22 & 1 & 1 & 5 & 15 & 22 & 44 \\
\hline $92-182$ & 1 & 4 & 4 & 5 & 14 & 1 & 1 & 3 & 4 & 9 & 23 \\
\hline $183+$ & 0 & 0 & 1 & 0 & 1 & 0 & 0 & 1 & 3 & 4 & 5 \\
\hline TOTAL & 13 & 29 & 83 & 108 & 233 & 14 & 32 & 101 & 249 & 396 & 629 \\
\hline
\end{tabular}

*Only those gender/age combinations with at least one absence appear in this table. 
Lawrence Livermore National Laboratory 2006

Absence Data

Appendix F. Distribution of the Number of Calendar Days Missed per Absence by Gender and Job Category*

Women

\begin{tabular}{|c|c|c|c|c|c|c|c|}
\hline \multirow{2}{*}{$\begin{array}{c}\# \\
\text { of } \\
\text { Calendar } \\
\text { Days }\end{array}$} & \multicolumn{6}{|c|}{ Job Category } & \multirow[b]{2}{*}{ TOTAL } \\
\hline & Professional & $\begin{array}{c}\text { Administrative } \\
\text { Support }\end{array}$ & $\begin{array}{l}\text { Technical } \\
\text { Support }\end{array}$ & Service & $\begin{array}{c}\text { Security } \\
\text { and } \\
\text { Fire }\end{array}$ & Crafts & \\
\hline$<15$ & 15 & 87 & 13 & 6 & 5 & 0 & 126 \\
\hline $15-28$ & 5 & 27 & 7 & 4 & 0 & 1 & 44 \\
\hline $29-42$ & 1 & 10 & 2 & 1 & 0 & 1 & 15 \\
\hline $43-56$ & 0 & 8 & 2 & 0 & 0 & 1 & 11 \\
\hline $57-91$ & 1 & 17 & 1 & 1 & 0 & 2 & 22 \\
\hline $92-182$ & 0 & 11 & 2 & 0 & 1 & 0 & 14 \\
\hline $183+$ & 0 & 0 & 0 & 1 & 0 & 0 & 1 \\
\hline TOTAL & 22 & 160 & 27 & 13 & 6 & 5 & 233 \\
\hline
\end{tabular}

Men

\begin{tabular}{|c|c|c|c|c|c|c|c|}
\hline \multirow{2}{*}{$\begin{array}{c}\# \\
\text { of } \\
\text { Calendar } \\
\text { Days }\end{array}$} & \multicolumn{6}{|c|}{ Job Category } & \multirow[b]{2}{*}{ TOTAI } \\
\hline & Professional & $\begin{array}{l}\text { Administrative } \\
\text { Support }\end{array}$ & $\begin{array}{l}\text { Technical } \\
\text { Support }\end{array}$ & Service & $\begin{array}{c}\text { Security } \\
\text { and } \\
\text { Fire }\end{array}$ & Crafts & \\
\hline$<15$ & 86 & 73 & 45 & 11 & 18 & 17 & 250 \\
\hline $15-28$ & 23 & 26 & 7 & 4 & 1 & 9 & 70 \\
\hline $29-42$ & 7 & 4 & 6 & 1 & 0 & 2 & 20 \\
\hline $43-56$ & 4 & 7 & 1 & 2 & 2 & 5 & 21 \\
\hline $57-91$ & 5 & 3 & 7 & 3 & 1 & 3 & 22 \\
\hline $92-182$ & 1 & 2 & 4 & 1 & 0 & 1 & 9 \\
\hline $183+$ & 1 & 2 & 0 & 0 & 0 & 1 & 4 \\
\hline TOTAL & 127 & 117 & 70 & 22 & 22 & 38 & 396 \\
\hline
\end{tabular}

*Only those gender/job category combinations with at least one absence appear in this table. 


\section{Lawrence Livermore National Laboratory 2006}

Absence Data

Appendix G. Number of Diagnoses in Each Diagnostic Category by Gender and Age*

\begin{tabular}{|c|c|c|c|c|c|c|}
\hline & & \multicolumn{5}{|c|}{ Women } \\
\hline & & \multicolumn{4}{|c|}{ Age Group } & \multirow[b]{2}{*}{ TOTAL } \\
\hline & & $16-29$ & $30-39$ & $40-49$ & $50+$ & \\
\hline Diagnostic Category & ICD-9-CM Code & \multirow[b]{2}{*}{2} & \multirow[b]{2}{*}{1} & \multirow[b]{2}{*}{3} & \multirow[b]{2}{*}{7} & \multirow[b]{2}{*}{13} \\
\hline INFECTIOUS \& PARASITIC DISEASES (DIS) & 001-139 & & & & & \\
\hline -Intestinal Infectious Dis & 001-009 & 0 & 0 & 0 & 1 & 1 \\
\hline -Other Bacterial Dis & 030-041 & 2 & 1 & 0 & 1 & 4 \\
\hline -Viral Dis with Exanthem & 050-057 & 0 & 0 & 0 & 2 & 2 \\
\hline -Other Viral Dis \& Chlamydiae & 070-079 & 0 & 0 & 3 & 3 & 6 \\
\hline -Mycoses & 110-118 & 0 & 0 & 0 & 0 & 0 \\
\hline -Other Infections \& Parasitic Dis & $130-136$ & 0 & 0 & 0 & 0 & 0 \\
\hline MALIGNANT NEOPLASMS & $140-208,230-234$ & 0 & 0 & 2 & 3 & 5 \\
\hline -Lip, Oral Cavity, Pharynx & 140-149 & 0 & 0 & 0 & 0 & 0 \\
\hline -Digestive \& Peritoneal & 150-159 & 0 & 0 & 0 & 0 & 0 \\
\hline -Bone, Connective Tissue, Skin & $170-173,176$ & 0 & 0 & 0 & 0 & 0 \\
\hline -Breast & $174-175$ & 0 & 0 & 1 & 3 & 4 \\
\hline -Genitourinary & 179-189 & 0 & 0 & 1 & 0 & 1 \\
\hline -Lymphatic \& Hematopoietic & $200-208$ & 0 & 0 & 0 & 0 & 0 \\
\hline BENIGN \& UNCERTAIN NEOPLASMS & 210-229, 235-239 & 1 & 0 & 5 & 4 & 10 \\
\hline ENDOCRINE/METABOLIC/IMMUNITY & 240-279 & 0 & 0 & 5 & 12 & 17 \\
\hline -Thyroid Gland Disorders & $240-246$ & 0 & 0 & 0 & 1 & 1 \\
\hline -Other Endocrine Gland Dis & $250-259$ & 0 & 0 & 0 & 1 & 1 \\
\hline -Other Metabolic \& Immunity Disorders & 270-279 & 0 & 0 & 5 & 10 & 15 \\
\hline MENTAL DISORDERS & 290-319 & 3 & 0 & 3 & 1 & 7 \\
\hline -Psychoses & 290-299 & 1 & 0 & 0 & 0 & 1 \\
\hline -Non-Psychotic Disorders & $300-302,306-316$ & 2 & 0 & 3 & 1 & 6 \\
\hline NERVOUS SYSTEM (NS) \& SENSE ORGANS & $320-389$ & 1 & 1 & 5 & 8 & 15 \\
\hline -Inflammatory Dis of Central NS & $320-326$ & 0 & 0 & 0 & 0 & 0 \\
\hline -Hereditary/Degenerative Central NS Dis & 330-337 & 0 & 0 & 0 & 0 & 0 \\
\hline -Other Disorders of Central NS & $340-349$ & 0 & 0 & 1 & 2 & 3 \\
\hline -Disorders of Peripheral NS & $350-359$ & 0 & 0 & 3 & 3 & 6 \\
\hline -Disorders of Eye & $360-379$ & 0 & 0 & 0 & 2 & 2 \\
\hline -Dis of Ear \& Mastoid & 380-389 & 1 & 1 & 1 & 1 & 4 \\
\hline CIRCULATORY SYSTEM & $390-459$ & 0 & 0 & 4 & 5 & 9 \\
\hline -Hypertensive Dis & 401-405 & 0 & 0 & 0 & 0 & 0 \\
\hline -Ischemic Heart Dis & $410-414$ & 0 & 0 & 0 & 1 & 1 \\
\hline -Dis of Pulmonary Circulation & $415-417$ & 0 & 0 & 0 & 1 & 1 \\
\hline -Other Heart Dis & $420-429$ & 0 & 0 & 0 & 1 & 1 \\
\hline -Cerebrovascular Dis & $430-438$ & 0 & 0 & 0 & 2 & 2 \\
\hline -Dis of Arteries \& Capillaries & $440-448$ & 0 & 0 & 1 & 0 & 1 \\
\hline -Dis of Veins, Lymphatics, Other & 451-459 & 0 & 0 & 3 & 0 & 3 \\
\hline
\end{tabular}

(Continued)

*Only those diagnostic categories and gender/age combinations with at least one occurrence appear in this table. 


\section{Lawrence Livermore National Laboratory 2006}

Absence Data

Appendix G. Number of Diagnoses in Each Diagnostic Category by Gender and Age*

\begin{tabular}{|c|c|c|c|c|c|c|c|}
\hline & & \multicolumn{5}{|c|}{ Men } & \multirow[b]{3}{*}{ TOTAL } \\
\hline & & \multicolumn{4}{|c|}{ Age Group } & \multirow[b]{2}{*}{ TOTAL } & \\
\hline & & $16-29$ & 30 - 39 & $40-49$ & $50+$ & & \\
\hline Diagnostic Category & ICD-9-CM Code & & & & & & \\
\hline INFECTIOUS \& PARASITIC DISEASES (DIS) & 001-139 & 0 & 3 & 6 & 11 & 20 & 33 \\
\hline -Intestinal Infectious Dis & 001-009 & 0 & 0 & 0 & 2 & 2 & 3 \\
\hline -Other Bacterial Dis & 030-041 & 0 & 1 & 1 & 1 & 3 & 7 \\
\hline -Viral Dis with Exanthem & 050-057 & 0 & 2 & 0 & 2 & 4 & 6 \\
\hline -Other Viral Dis \& Chlamydiae & 070-079 & 0 & 0 & 5 & 4 & 9 & 15 \\
\hline -Mycoses & $110-118$ & 0 & 0 & 0 & 1 & 1 & 1 \\
\hline -Other Infections \& Parasitic Dis & $130-136$ & 0 & 0 & 0 & 1 & 1 & 1 \\
\hline MALIGNANT NEOPLASMS & 140-208, 230-234 & 0 & 0 & 3 & 12 & 15 & 20 \\
\hline -Lip, Oral Cavity, Pharynx & $140-149$ & 0 & 0 & 1 & 0 & 1 & 1 \\
\hline -Digestive \& Peritoneal & $150-159$ & 0 & 0 & 0 & 1 & 1 & 1 \\
\hline -Bone, Connective Tissue, Skin & $170-173,176$ & 0 & 0 & 1 & 1 & 2 & 2 \\
\hline -Breast & 174-175 & 0 & 0 & 0 & 0 & 0 & 4 \\
\hline -Genitourinary & 179-189 & 0 & 0 & 1 & 8 & 9 & 10 \\
\hline -Lymphatic \& Hematopoietic & $200-208$ & 0 & 0 & 0 & 2 & 2 & 2 \\
\hline BENIGN \& UNCERTAIN NEOPLASMS & 210-229, 235-239 & 0 & 0 & 0 & 8 & 8 & 18 \\
\hline ENDOCRINE/METABOLIC/IMMUNITY & $240-279$ & 1 & 0 & 3 & 6 & 10 & 27 \\
\hline -Thyroid Gland Disorders & $240-246$ & 0 & 0 & 0 & 1 & 1 & 2 \\
\hline -Other Endocrine Gland Dis & $250-259$ & 0 & 0 & 0 & 1 & 1 & 2 \\
\hline -Other Metabolic \& Immunity Disorders & $270-279$ & 1 & 0 & 3 & 4 & 8 & 23 \\
\hline MENTAL DISORDERS & $290-319$ & 1 & 0 & 0 & 3 & 4 & 11 \\
\hline -Psychoses & $290-299$ & 0 & 0 & 0 & 0 & 0 & 1 \\
\hline -Non-Psychotic Disorders & $300-302,306-316$ & 1 & 0 & 0 & 3 & 4 & 10 \\
\hline NERVOUS SYSTEM (NS) \& SENSE ORGANS & $320-389$ & 1 & 2 & 7 & 18 & 28 & 43 \\
\hline -Inflammatory Dis of Central NS & $320-326$ & 0 & 0 & 1 & 0 & 1 & 1 \\
\hline -Hereditary/Degenerative Central NS Dis & $330-337$ & 0 & 0 & 0 & 1 & 1 & 1 \\
\hline -Other Disorders of Central NS & $340-349$ & 0 & 0 & 0 & 0 & 0 & 3 \\
\hline -Disorders of Peripheral NS & $350-359$ & 0 & 1 & 2 & 7 & 10 & 16 \\
\hline -Disorders of Eye & $360-379$ & 1 & 1 & 1 & 9 & 12 & 14 \\
\hline -Dis of Ear \& Mastoid & $380-389$ & 0 & 0 & 3 & 1 & 4 & 8 \\
\hline CIRCULATORY SYSTEM & $390-459$ & 0 & 0 & 7 & 24 & 31 & 40 \\
\hline -Hypertensive Dis & $401-405$ & 0 & 0 & 2 & 1 & 3 & 3 \\
\hline -Ischemic Heart Dis & $410-414$ & 0 & 0 & 1 & 6 & 7 & 8 \\
\hline -Dis of Pulmonary Circulation & $415-417$ & 0 & 0 & 0 & 0 & 0 & 1 \\
\hline -Other Heart Dis & $420-429$ & 0 & 0 & 1 & 4 & 5 & $\overline{6}$ \\
\hline -Cerebrovascular Dis & $430-438$ & 0 & 0 & 1 & 6 & 7 & 9 \\
\hline -Dis of Arteries \& Capillaries & $440-448$ & 0 & 0 & 1 & 3 & 4 & 5 \\
\hline -Dis of Veins, Lymphatics, Other & $451-459$ & 0 & 0 & 1 & 4 & 5 & 8 \\
\hline
\end{tabular}

(Continued)

*Only those diagnostic categories and gender/age combinations with at least one occurrence appear in this table. 


\section{Lawrence Livermore National Laboratory 2006}

Absence Data

Appendix G. Number of Diagnoses in Each Diagnostic Category by Gender and Age*

\begin{tabular}{|c|c|c|c|c|c|c|}
\hline & & \multicolumn{5}{|c|}{ Women } \\
\hline & & \multicolumn{4}{|c|}{ Age Group } & \multirow[b]{2}{*}{ TOTAL } \\
\hline & & $16-29$ & $30-39$ & $40-49$ & $50+$ & \\
\hline Diagnostic Category & $\begin{array}{l}\text { ICD-9-CM } \\
\text { Code }\end{array}$ & & & & & \\
\hline RESPIRATORY SYSTEM & 460-519 & 5 & 4 & 14 & 24 & 47 \\
\hline -Acute Respiratory Infections & $460-466$ & 3 & 3 & 7 & 12 & 25 \\
\hline -Other Dis Upper Respiratory Tract & $470-478$ & 1 & 1 & 2 & 1 & 5 \\
\hline -Pneumonia \& Influenza & $480-487$ & 1 & 0 & 2 & 6 & 9 \\
\hline -Chronic Obstructive Dis & $490-496$ & 0 & 0 & 3 & 4 & 7 \\
\hline -Other Respiratory Dis & $510-519$ & 0 & 0 & 0 & 1 & 1 \\
\hline DIGESTIVE SYSTEM & $520-579$ & 1 & 7 & 8 & 14 & 30 \\
\hline -Oral Cavity, Saliva Glands, Jaw & $520-529$ & 0 & 0 & 0 & 0 & 0 \\
\hline -Esophagus, Stomach, Duodenum & $530-537$ & 0 & 0 & 0 & 1 & 1 \\
\hline -Appendicitis & $540-543$ & 1 & 2 & 0 & 0 & 3 \\
\hline -Hernia & $550-553$ & 0 & 0 & 1 & 3 & 4 \\
\hline -Enteritis, Colitis & $555-558$ & 0 & 0 & 2 & 0 & 2 \\
\hline -Other Intestinal Dis & $560-569$ & 0 & 0 & 2 & 5 & 7 \\
\hline -Other Digestive Dis & $570-579$ & 0 & 5 & 3 & 5 & 13 \\
\hline GENITOURINARY SYSTEM & $580-629$ & 0 & 6 & 16 & 20 & 42 \\
\hline -Nephritis, Nephrosis & $580-589$ & 0 & 2 & 0 & 2 & 4 \\
\hline -Other Urinary Dis & $590-599$ & 0 & 0 & 2 & 6 & 8 \\
\hline -Male Genital Organ Dis & $600-608$ & 0 & 0 & 0 & 0 & 0 \\
\hline -Breast Disorders & $610-611$ & 0 & 2 & 2 & 4 & 8 \\
\hline -Pelvic Inflammatory Dis & 614-616 & 0 & 0 & 1 & 0 & 1 \\
\hline -Other Female Disorders & 617-629 & 0 & 2 & 11 & 8 & 21 \\
\hline PREGNANCY \& CHILDBIRTH & $630-677$ & 0 & 1 & 0 & 0 & 1 \\
\hline -Pregnancy with Abortive Outcome & 634-639 & 0 & 1 & 0 & 0 & 1 \\
\hline SKIN \& SUBCUTANEOUS TISSUE & $680-709$ & 0 & 2 & 2 & 9 & 13 \\
\hline -Infections & $680-686$ & 0 & 1 & 1 & 5 & 7 \\
\hline -Other Inflammatory Conditions & $690-698$ & 0 & 0 & 0 & 0 & 0 \\
\hline -Other & 700-709 & 0 & 1 & 1 & 4 & 6 \\
\hline MUSCULOSKELETAL \& CONNECTIVE TISSUE & $710-739$ & 1 & 8 & 14 & 17 & 40 \\
\hline -Arthropathies & $710-719$ & 1 & 2 & 6 & 6 & 15 \\
\hline -Dorsopathies & 720-724 & 0 & 5 & 4 & 3 & 12 \\
\hline -Rheumatism, Excluding Back & 725-729 & 0 & 1 & 4 & 7 & 12 \\
\hline -Other Dis \& Acquired Deformities & $730-739$ & 0 & 0 & 0 & 1 & 1 \\
\hline CONGENITAL ANOMALIES & $740-759$ & 0 & 0 & 0 & 2 & 2 \\
\hline SYMPTOMS, SIGNS, \& ILL-DEFINED CONDITIONS & 780-799 & 0 & 0 & 4 & 6 & 10 \\
\hline -Symptoms & $780-789$ & 0 & 0 & 4 & 6 & 10 \\
\hline -Non-Specific Abnormal Findings & $790-796$ & 0 & 0 & 0 & 0 & 0 \\
\hline
\end{tabular}

(Continued)

*Only those diagnostic categories and gender/age combinations with at least one occurrence appear in this table. 


\section{Lawrence Livermore National Laboratory 2006}

Absence Data

Appendix G. Number of Diagnoses in Each Diagnostic Category by Gender and Age*

\begin{tabular}{|c|c|c|c|c|c|c|c|}
\hline & & \multicolumn{5}{|c|}{ Men } & \multirow[b]{3}{*}{ TOTAL } \\
\hline & & \multicolumn{4}{|c|}{ Age Group } & \multirow[b]{2}{*}{ TOTAL } & \\
\hline & & $16-29$ & 30 - 39 & $40-49$ & $50+$ & & \\
\hline Diagnostic Category & $\begin{array}{l}\text { ICD-9-CM } \\
\text { Code }\end{array}$ & & & & & & \\
\hline RESPIRATORY SYSTEM & $460-519$ & 2 & 8 & 16 & 43 & 69 & 116 \\
\hline -Acute Respiratory Infections & $460-466$ & 1 & 4 & 8 & 18 & 31 & 56 \\
\hline -Other Dis Upper Respiratory Tract & $470-478$ & 0 & 1 & 4 & 5 & 10 & 15 \\
\hline -Pneumonia \& Influenza & $480-487$ & 1 & 3 & 3 & 15 & 22 & 31 \\
\hline -Chronic Obstructive Dis & $490-496$ & 0 & 0 & 1 & 4 & 5 & 12 \\
\hline -Other Respiratory Dis & $510-519$ & 0 & 0 & 0 & 1 & 1 & 2 \\
\hline DIGESTIVE SYSTEM & $520-579$ & 3 & 2 & 11 & 31 & 47 & 77 \\
\hline -Oral Cavity, Saliva Glands, Jaw & $520-529$ & 1 & 0 & 1 & 1 & 3 & 3 \\
\hline -Esophagus, Stomach, Duodenum & $530-537$ & 0 & 0 & 0 & 1 & 1 & 2 \\
\hline -Appendicitis & $540-543$ & 0 & 0 & 3 & 1 & 4 & 7 \\
\hline -Hernia & $550-553$ & 1 & 0 & 2 & 10 & 13 & 17 \\
\hline -Enteritis, Colitis & $555-558$ & 1 & 1 & 0 & 2 & 4 & 6 \\
\hline -Other Intestinal Dis & $560-569$ & 0 & 0 & 2 & 8 & 10 & 17 \\
\hline -Other Digestive Dis & $570-579$ & 0 & 1 & 3 & 8 & 12 & 25 \\
\hline GENITOURINARY SYSTEM & $580-629$ & 0 & 1 & 6 & 8 & 15 & 57 \\
\hline -Nephritis, Nephrosis & $580-589$ & 0 & 0 & 0 & 0 & 0 & 4 \\
\hline -Other Urinary Dis & $590-599$ & 0 & 0 & 6 & 7 & 13 & 21 \\
\hline -Male Genital Organ Dis & $600-608$ & 0 & 1 & 0 & 1 & 2 & 2 \\
\hline -Breast Disorders & $610-611$ & 0 & 0 & 0 & 0 & 0 & 8 \\
\hline -Pelvic Inflammatory Dis & 614-616 & 0 & 0 & 0 & 0 & 0 & 1 \\
\hline -Other Female Disorders & 617-629 & 0 & 0 & 0 & 0 & 0 & 21 \\
\hline PREGNANCY \& CHILDBIRTH & 630-677 & 0 & 0 & 0 & 0 & 0 & 1 \\
\hline -Pregnancy with Abortive Outcome & 634-639 & 0 & 0 & 0 & 0 & 0 & 1 \\
\hline SKIN \& SUBCUTANEOUS TISSUE & $680-709$ & 0 & 2 & 4 & 5 & 11 & 24 \\
\hline -Infections & $680-686$ & 0 & 0 & 2 & 2 & 4 & 11 \\
\hline -Other Inflammatory Conditions & 690-698 & 0 & 1 & 0 & 0 & 1 & 1 \\
\hline -Other & 700-709 & 0 & 1 & 2 & 3 & 6 & 12 \\
\hline MUSCULOSKELETAL \& CONNECTIVE TISSUE & 710-739 & 2 & 6 & 27 & 49 & 84 & 124 \\
\hline -Arthropathies & $710-719$ & 1 & 1 & 9 & 19 & 30 & 45 \\
\hline -Dorsopathies & $720-724$ & 1 & 5 & 13 & 21 & 40 & 52 \\
\hline -Rheumatism, Excluding Back & 725-729 & 0 & 0 & 4 & 8 & 12 & 24 \\
\hline -Other Dis \& Acquired Deformities & 730-739 & 0 & 0 & 1 & 1 & 2 & 3 \\
\hline CONGENITAL ANOMALIES & $740-759$ & 0 & 0 & 0 & 1 & 1 & 3 \\
\hline SYMPTOMS, SIGNS, \& ILL-DEFINED CONDITIONS & 780-799 & 2 & 1 & 7 & 15 & 25 & 35 \\
\hline -Symptoms & 780-789 & 2 & 1 & 7 & 14 & 24 & 34 \\
\hline -Non-Specific Abnormal Findings & $790-796$ & 0 & 0 & 0 & 1 & 1 & 1 \\
\hline
\end{tabular}

(Continued)

*Only those diagnostic categories and gender/age combinations with at least one occurrence appear in this table. 


\section{Lawrence Livermore National Laboratory 2006}

Absence Data

Appendix G. Number of Diagnoses in Each Diagnostic Category by Gender and Age*

\begin{tabular}{|c|c|c|c|c|c|c|}
\hline & & \multicolumn{5}{|c|}{ Women } \\
\hline & & \multicolumn{4}{|c|}{ Age Group } & \multirow[b]{2}{*}{ TOTAL } \\
\hline & & $16-29$ & $30-39$ & 40 - 49 & $50+$ & \\
\hline Diagnostic Category & $\begin{array}{l}\text { ICD-9-CM } \\
\text { Code }\end{array}$ & & & & & \\
\hline INJURY \& POISONING & 800-999 & 1 & 2 & 9 & 9 & 21 \\
\hline -Fracture - Skull & $800-804$ & 0 & 0 & 0 & 0 & 0 \\
\hline -Fracture - Neck, Trunk & $805-809$ & 0 & 0 & 0 & 0 & 0 \\
\hline -Fracture - Upper Limb & 810-819 & 0 & 0 & 0 & 2 & 2 \\
\hline -Fracture - Lower Limb & $820-829$ & 0 & 0 & 2 & 3 & 5 \\
\hline -Dislocation & $830-839$ & 0 & 0 & 1 & 1 & 2 \\
\hline -Sprains \& Strains - Back & 846-847 & 0 & 0 & 0 & 0 & 0 \\
\hline -Sprains \& Strains - Other & $840-845,848$ & 1 & 1 & 3 & 1 & 6 \\
\hline -Internal Injury - Thorax, Abdomen, Pelvis & $860-869$ & 0 & 0 & 0 & 0 & 0 \\
\hline -Open Wound - Head, Neck, Trunk & 870-879 & 0 & 0 & 0 & 0 & 0 \\
\hline -Open Wound - Upper Limb & 880-887 & 0 & 0 & 0 & 0 & 0 \\
\hline -Late Effects of Accident & 905-909 & 0 & 0 & 0 & 0 & 0 \\
\hline -Superficial Injury & 910-919 & 0 & 0 & 1 & 0 & 1 \\
\hline -Contusion & 920-924 & 0 & 0 & 0 & 0 & 0 \\
\hline -Burns & 940-949 & 0 & 0 & 0 & 0 & 0 \\
\hline -Complications \& Unspecified Injuries & 958-959 & 0 & 0 & 1 & 0 & 1 \\
\hline -Unspecified Effects - External Causes & 990-995 & 0 & 0 & 0 & 0 & 0 \\
\hline -Complications of Surgical/Medical Care & 996-999 & 0 & 1 & 1 & 2 & 4 \\
\hline HEALTH STATUS/HEALTH SERVICE CONTACT & V01-V82 & 0 & 3 & 3 & 2 & 8 \\
\hline -Personal \& Family History & V10-V19 & 0 & 0 & 0 & 0 & 0 \\
\hline -Health Services Reproduction/Development & V20-V29 & 0 & 2 & 0 & 0 & 2 \\
\hline -Specific Procedures/Aftercare & V50-V59 & 0 & 1 & 3 & 1 & 5 \\
\hline -Other Circumstances & V60-V69 & 0 & 0 & 0 & 0 & 0 \\
\hline -Examination \& Investigation & V70-V82 & 0 & 0 & 0 & 1 & 1 \\
\hline
\end{tabular}

*Only those diagnostic categories and gender/age combinations with at least one occurrence appear in this table. 


\section{Lawrence Livermore National Laboratory 2006}

Absence Data

Appendix G. Number of Diagnoses in Each Diagnostic Category by Gender and Age*

\begin{tabular}{|c|c|c|c|c|c|c|c|}
\hline & & \multicolumn{5}{|c|}{ Men } & \multirow[b]{3}{*}{ TOTAL } \\
\hline & & \multicolumn{4}{|c|}{ Age Group } & \multirow[b]{2}{*}{ TOTAL } & \\
\hline & & $16-29$ & 30 - 39 & $40-49$ & $50+$ & & \\
\hline Diagnostic Category & \begin{tabular}{|l|} 
ICD-9-CM \\
Code
\end{tabular} & & & & & & \\
\hline INJURY \& POISONING & $800-999$ & 2 & 9 & 26 & 51 & 88 & 109 \\
\hline -Fracture - Skull & $800-804$ & 0 & 1 & 0 & 1 & 2 & 2 \\
\hline -Fracture - Neck, Trunk & $805-809$ & 0 & 0 & 1 & 2 & 3 & 3 \\
\hline -Fracture - Upper Limb & 810-819 & 0 & 0 & 4 & 1 & 5 & 7 \\
\hline -Fracture - Lower Limb & $820-829$ & 0 & 2 & 5 & 5 & 12 & 17 \\
\hline -Dislocation & $830-839$ & 0 & 2 & 2 & 11 & 15 & 17 \\
\hline -Sprains \& Strains - Back & 846-847 & 1 & 1 & 2 & 6 & 10 & 10 \\
\hline -Sprains \& Strains - Other & $840-845,848$ & 1 & 3 & 5 & 11 & 20 & 26 \\
\hline -Internal Injury - Thorax, Abdomen, Pelvis & $860-869$ & 0 & 0 & 1 & 0 & 1 & 1 \\
\hline -Open Wound - Head, Neck, Trunk & $870-879$ & 0 & 0 & 1 & 1 & 2 & 2 \\
\hline -Open Wound - Upper Limb & $880-887$ & 0 & 0 & 0 & 3 & 3 & 3 \\
\hline -Late Effects of Accident & 905-909 & 0 & 0 & 1 & 0 & 1 & 1 \\
\hline -Superficial Injury & 910-919 & 0 & 0 & 0 & 0 & 0 & 1 \\
\hline -Contusion & $920-924$ & 0 & 0 & 2 & 1 & 3 & 3 \\
\hline -Burns & 940-949 & 0 & 0 & 0 & 1 & 1 & 1 \\
\hline -Complications \& Unspecified Injuries & 958-959 & 0 & 0 & 2 & 6 & 8 & 9 \\
\hline -Unspecified Effects - External Causes & 990-995 & 0 & 0 & 0 & 1 & 1 & 1 \\
\hline -Complications of Surgical/Medical Care & 996-999 & 0 & 0 & 0 & 1 & 1 & 5 \\
\hline HEALTH STATUS/HEALTH SERVICE CONTACT & V01-V82 & 0 & 4 & 1 & 2 & 7 & 15 \\
\hline -Personal \& Family History & V10-V19 & 0 & 0 & 0 & 1 & 1 & 1 \\
\hline -Health Services Reproduction/Development & V20-V29 & 0 & 2 & 1 & 0 & 3 & 5 \\
\hline -Specific Procedures/Aftercare & V50-V59 & 0 & 2 & 0 & 0 & 2 & 7 \\
\hline -Other Circumstances & V60-V69 & 0 & 0 & 0 & 1 & 1 & 1 \\
\hline -Examination \& Investigation & V70-V82 & 0 & 0 & 0 & 0 & 0 & 1 \\
\hline
\end{tabular}

\begin{tabular}{|c|c|c|c|c|c|c|c|c|c|c|c|}
\hline & \multicolumn{5}{|c|}{ Women } & \multicolumn{5}{|c|}{ Men } & \multirow[b]{3}{*}{ TOTAL } \\
\hline & \multicolumn{4}{|c|}{ Age Group } & \multirow[b]{2}{*}{ TOTAL } & \multicolumn{4}{|c|}{ Age Group } & \multirow[b]{2}{*}{ TOTAL } & \\
\hline & $16-29$ & $30-39$ & $40-49$ & $50+$ & & $16-29$ & $30-39$ & $40-49$ & $50+$ & & \\
\hline $\begin{array}{l}\text { Diagnostic } \\
\text { Category }\end{array}$ & & & & & & & & & & & \\
\hline Total & 15 & 35 & 97 & 143 & 290 & 14 & 38 & 124 & 287 & 463 & 753 \\
\hline
\end{tabular}

*Only those diagnostic categories and gender/age combinations with at least one occurrence appear in this table. 
Lawrence Livermore National Laboratory 2006

Absence Data

Appendix H. Total Number of Calendar Days Absent in Each Diagnostic Category by Gender and Age*

\begin{tabular}{|c|c|c|c|c|c|c|}
\hline & & \multicolumn{5}{|c|}{ Women } \\
\hline & & \multicolumn{4}{|c|}{ Age Group } & \multirow[b]{2}{*}{ TOTAL } \\
\hline & & $16-29$ & $30-39$ & $40-49$ & $50+$ & \\
\hline Diagnostic Category & ICD-9-CM Code & \multirow[b]{2}{*}{19} & \multirow[b]{2}{*}{13} & \multirow[b]{2}{*}{163} & \multirow[b]{2}{*}{119} & \multirow[b]{2}{*}{314} \\
\hline INFECTIOUS \& PARASITIC DISEASES (DIS) & 001-139 & & & & & \\
\hline MALIGNANT NEOPLASMS & 140-208, 230-234 & 0 & 0 & 187 & 50 & 237 \\
\hline BENIGN \& UNCERTAIN NEOPLASMS & 210-229, 235-239 & 15 & 0 & 287 & 201 & 503 \\
\hline ENDOCRINE/METABOLIC/IMMUNITY & $240-279$ & 0 & 0 & 108 & 408 & 516 \\
\hline MENTAL DISORDERS & $290-319$ & 186 & 0 & 298 & 10 & 494 \\
\hline NERVOUS SYSTEM (NS) \& SENSE ORGANS & $320-389$ & 5 & 17 & 104 & 135 & 261 \\
\hline CIRCULATORY SYSTEM & $390-459$ & 0 & 0 & 53 & 99 & 152 \\
\hline RESPIRATORY SYSTEM & $460-519$ & 33 & 35 & 145 & 210 & 423 \\
\hline DIGESTIVE SYSTEM & $520-579$ & 10 & 142 & 111 & 459 & 722 \\
\hline GENITOURINARY SYSTEM & $580-629$ & 0 & 123 & 327 & 485 & 935 \\
\hline PREGNANCY \& CHILDBIRTH & $630-677$ & 0 & 5 & 0 & 0 & 5 \\
\hline SKIN \& SUBCUTANEOUS TISSUE & $680-709$ & 0 & 38 & 80 & 241 & 359 \\
\hline MUSCULOSKELETAL \& CONNECTIVE TISSUE & $710-739$ & 2 & 517 & 509 & 777 & 1,805 \\
\hline CONGENITAL ANOMALIES & $740-759$ & 0 & 0 & 0 & 38 & 38 \\
\hline SYMPTOMS, SIGNS, \& ILL-DEFINED CONDITIONS & 780-799 & 0 & 0 & 63 & 43 & 106 \\
\hline INJURY \& POISONING & $800-999$ & 5 & 76 & 238 & 365 & 684 \\
\hline
\end{tabular}

\footnotetext{
*Absences with >1 ICD-9-CM code in the same diagnostic category were counted only once. Only those diagnostic categories and gender/age combinations with at least one occurrence appear in this table.
} 
Lawrence Livermore National Laboratory 2006

Absence Data

Appendix H. Total Number of Calendar Days Absent in Each Diagnostic Category by Gender and Age*

\begin{tabular}{|c|c|c|c|c|c|c|c|}
\hline & & \multicolumn{5}{|c|}{ Men } & \multirow[b]{3}{*}{ TOTAL } \\
\hline & & \multicolumn{4}{|c|}{ Age Group } & \multirow[b]{2}{*}{ TOTAL } & \\
\hline & & $16-29$ & $30-39$ & $40-49$ & $\mathbf{5 0 +}$ & & \\
\hline Diagnostic Category & ICD-9-CM Code & \multirow[b]{2}{*}{0} & \multirow[b]{2}{*}{20} & \multirow[b]{2}{*}{61} & \multirow[b]{2}{*}{172} & \multirow[b]{2}{*}{253} & \multirow[b]{2}{*}{567} \\
\hline INFECTIOUS \& PARASITIC DISEASES (DIS) & $001-139$ & & & & & & \\
\hline MALIGNANT NEOPLASMS & $140-208,230-234$ & 0 & 0 & 185 & 659 & 844 & 1,081 \\
\hline BENIGN \& UNCERTAIN NEOPLASMS & 210-229, 235-239 & 0 & 0 & 0 & 240 & 240 & 743 \\
\hline ENDOCRINE/METABOLIC/IMMUNITY & $240-279$ & 18 & 0 & 15 & 121 & 154 & 670 \\
\hline MENTAL DISORDERS & $290-319$ & 111 & 0 & 0 & 24 & 135 & 629 \\
\hline NERVOUS SYSTEM (NS) \& SENSE ORGANS & $320-389$ & 5 & 105 & 109 & 455 & 674 & 935 \\
\hline CIRCULATORY SYSTEM & $390-459$ & 0 & 0 & 287 & 584 & 871 & 1,023 \\
\hline RESPIRATORY SYSTEM & $460-519$ & 11 & 44 & 107 & 489 & 651 & 1,074 \\
\hline DIGESTIVE SYSTEM & $520-579$ & 66 & 53 & 150 & 495 & 764 & 1,486 \\
\hline GENITOURINARY SYSTEM & $580-629$ & 0 & 7 & 50 & 85 & 142 & 1,077 \\
\hline PREGNANCY \& CHILDBIRTH & $630-677$ & 0 & 0 & 0 & 0 & 0 & 5 \\
\hline SKIN \& SUBCUTANEOUS TISSUE & 680-709 & 0 & 13 & 68 & 92 & 173 & 532 \\
\hline MUSCULOSKELETAL \& CONNECTIVE TISSUE & 710-739 & 27 & 241 & 725 & 1,158 & 2,151 & 3,956 \\
\hline CONGENITAL ANOMALIES & $740-759$ & 0 & 0 & 0 & 1 & 1 & 39 \\
\hline SYMPTOMS, SIGNS, \& ILL-DEFINED CONDITIONS & 780-799 & 19 & 14 & 108 & 297 & 438 & 544 \\
\hline INJURY \& POISONING & $800-999$ & 87 & 139 & 482 & 1,189 & 1,897 & 2,581 \\
\hline
\end{tabular}

\footnotetext{
*Absences with >1 ICD-9-CM code in the same diagnostic category were counted only once. Only those diagnostic categories and gender/age combinations with at least one occurrence appear in this table.
} 


\section{Lawrence Livermore National Laboratory 2006}

\section{Absence Data}

Appendix I. Number of Diagnoses in Each Diagnostic Category by Gender and Job Category*

\begin{tabular}{|c|c|c|c|c|c|c|c|c|}
\hline & & \multicolumn{7}{|c|}{ Women } \\
\hline & & \multicolumn{6}{|c|}{ Job Category } & \multirow[b]{2}{*}{ TOTAL } \\
\hline & & Professional & $\begin{array}{l}\text { Administrative } \\
\text { Support }\end{array}$ & $\begin{array}{l}\text { Technical } \\
\text { Support }\end{array}$ & Service & $\begin{array}{c}\text { Security } \\
\text { and } \\
\text { Fire }\end{array}$ & Crafts & \\
\hline Diagnostic Category & \begin{tabular}{|l|} 
ICD-9-CM \\
Code
\end{tabular} & & & & & & & \\
\hline $\begin{array}{l}\text { INFECTIOUS \& PARASITIC DISEASES } \\
\text { (DIS) }\end{array}$ & 001-139 & 1 & 9 & 1 & 1 & 1 & 0 & 13 \\
\hline -Intestinal Infectious Dis & 001-009 & 0 & 1 & 0 & 0 & 0 & 0 & 1 \\
\hline -Other Bacterial Dis & $030-041$ & 1 & 2 & 0 & 0 & 1 & 0 & 4 \\
\hline -Viral Dis with Exanthem & $050-057$ & 0 & 2 & 0 & 0 & 0 & 0 & 2 \\
\hline -Other Viral Dis \& Chlamydiae & 070-079 & 0 & 4 & 1 & 1 & 0 & 0 & 6 \\
\hline MALIGNANT NEOPLASMS & $\begin{array}{l}140-208 \\
230-234\end{array}$ & 3 & 1 & 1 & 0 & 0 & 0 & 5 \\
\hline -Breast & 174-175 & 3 & 1 & 0 & 0 & 0 & 0 & 4 \\
\hline -Genitourinary & 179-189 & 0 & 0 & 1 & 0 & 0 & 0 & 1 \\
\hline BENIGN \& UNCERTAIN NEOPLASMS & $\begin{array}{l}\text { 210-229, } \\
\text { 235-239 }\end{array}$ & 0 & 6 & 1 & 1 & 0 & 2 & 10 \\
\hline ENDOCRINE/METABOLIC/IMMUNITY & $240-279$ & 1 & 14 & 2 & 0 & 0 & 0 & 17 \\
\hline -Thyroid Gland Disorders & $240-246$ & 0 & 1 & 0 & 0 & 0 & 0 & 1 \\
\hline -Other Endocrine Gland Dis & $250-259$ & 0 & 1 & 0 & 0 & 0 & 0 & 1 \\
\hline -Other Metabolic \& Immunity Disorders & 270-279 & 1 & 12 & 2 & 0 & 0 & 0 & 15 \\
\hline MENTAL DISORDERS & 290-319 & 1 & 6 & 0 & 0 & 0 & 0 & 7 \\
\hline -Psychoses & $290-299$ & 0 & 1 & 0 & 0 & 0 & 0 & 1 \\
\hline -Non-Psychotic Disorders & $\begin{array}{l}300-302 \\
306-316\end{array}$ & 1 & 5 & 0 & 0 & 0 & 0 & 6 \\
\hline $\begin{array}{l}\text { NERVOUS SYSTEM (NS) \& SENSE } \\
\text { ORGANS }\end{array}$ & $320-389$ & 0 & 14 & 0 & 0 & 0 & 1 & 15 \\
\hline -Other Disorders of Central NS & $340-349$ & 0 & 3 & 0 & 0 & 0 & 0 & 3 \\
\hline -Disorders of Peripheral NS & $350-359$ & 0 & 5 & 0 & 0 & 0 & 1 & 6 \\
\hline -Disorders of Eye & $360-379$ & 0 & 2 & 0 & 0 & 0 & 0 & 2 \\
\hline -Dis of Ear \& Mastoid & $380-389$ & 0 & 4 & 0 & 0 & 0 & 0 & 4 \\
\hline CIRCULATORY SYSTEM & $390-459$ & 0 & 8 & 1 & 0 & 0 & 0 & 9 \\
\hline -Ischemic Heart Dis & $410-414$ & 0 & 1 & 0 & 0 & 0 & 0 & 1 \\
\hline -Dis of Pulmonary Circulation & 415-417 & 0 & 1 & 0 & 0 & 0 & 0 & 1 \\
\hline -Other Heart Dis & $420-429$ & 0 & 1 & 0 & 0 & 0 & 0 & 1 \\
\hline -Cerebrovascular Dis & $430-438$ & 0 & 2 & 0 & 0 & 0 & 0 & 2 \\
\hline -Dis of Arteries \& Capillaries & $440-448$ & 0 & 1 & 0 & 0 & 0 & 0 & 1 \\
\hline -Dis of Veins, Lymphatics, Other & 451-459 & 0 & 2 & 1 & 0 & 0 & 0 & 3 \\
\hline RESPIRATORY SYSTEM & $460-519$ & 5 & 28 & 4 & 7 & 1 & 2 & 47 \\
\hline -Acute Respiratory Infections & $460-466$ & 4 & 14 & 4 & 2 & 1 & 0 & 25 \\
\hline -Other Dis Upper Respiratory Tract & $470-478$ & 1 & 3 & 0 & 1 & 0 & 0 & 5 \\
\hline
\end{tabular}

(Continued)

*Only those diagnostic categories and gender/job category combinations with at least one occurrence appear in this table. 


\section{Lawrence Livermore National Laboratory 2006}

\section{Absence Data}

Appendix I. Number of Diagnoses in Each Diagnostic Category by Gender and Job Category*

\begin{tabular}{|c|c|c|c|c|c|c|c|c|}
\hline & & \multicolumn{7}{|c|}{ Women } \\
\hline & & \multicolumn{6}{|c|}{ Job Category } & \multirow[b]{2}{*}{ TOTAL } \\
\hline & & Professional & $\begin{array}{l}\text { Administrative } \\
\text { Support }\end{array}$ & $\begin{array}{l}\text { Technical } \\
\text { Support }\end{array}$ & Service & $\begin{array}{c}\text { Security } \\
\text { and } \\
\text { Fire }\end{array}$ & Crafts & \\
\hline Diagnostic Category & \begin{tabular}{|l|} 
ICD-9-CM \\
Code
\end{tabular} & & & & & & & \\
\hline -Pneumonia \& Influenza & $480-487$ & 0 & 5 & 0 & 3 & 0 & 1 & 9 \\
\hline -Chronic Obstructive Dis & $490-496$ & 0 & 6 & 0 & 0 & 0 & 1 & 7 \\
\hline -Other Respiratory Dis & $510-519$ & 0 & 0 & 0 & 1 & 0 & 0 & 1 \\
\hline DIGESTIVE SYSTEM & $520-579$ & 3 & 23 & 4 & 0 & 0 & 0 & 30 \\
\hline -Esophagus, Stomach, Duodenum & $530-537$ & 0 & 1 & 0 & 0 & 0 & 0 & 1 \\
\hline -Appendicitis & $540-543$ & 0 & 3 & 0 & 0 & 0 & 0 & 3 \\
\hline -Hernia & $550-553$ & 0 & 4 & 0 & 0 & 0 & 0 & 4 \\
\hline -Enteritis, Colitis & $555-558$ & 0 & 2 & 0 & 0 & 0 & 0 & 2 \\
\hline -Other Intestinal Dis & $560-569$ & 0 & 6 & 1 & 0 & 0 & 0 & 7 \\
\hline -Other Digestive Dis & $570-579$ & 3 & 7 & 3 & 0 & 0 & 0 & 13 \\
\hline GENITOURINARY SYSTEM & $580-629$ & 3 & 33 & 2 & 2 & 2 & 0 & 42 \\
\hline -Nephritis, Nephrosis & $580-589$ & 0 & 4 & 0 & 0 & 0 & 0 & 4 \\
\hline -Other Urinary Dis & $590-599$ & 2 & 2 & 0 & 2 & 2 & 0 & 8 \\
\hline -Breast Disorders & $610-611$ & 1 & 7 & 0 & 0 & 0 & 0 & 8 \\
\hline -Pelvic Inflammatory Dis & 614-616 & 0 & 1 & 0 & 0 & 0 & 0 & 1 \\
\hline -Other Female Disorders & $617-629$ & 0 & 19 & 2 & 0 & 0 & 0 & 21 \\
\hline PREGNANCY \& CHILDBIRTH & $630-677$ & 0 & 0 & 1 & 0 & 0 & 0 & 1 \\
\hline -Pregnancy with Abortive Outcome & $634-639$ & 0 & 0 & 1 & 0 & 0 & 0 & 1 \\
\hline SKIN \& SUBCUTANEOUS TISSUE & $680-709$ & 3 & 7 & 2 & 1 & 0 & 0 & 13 \\
\hline -Infections & $680-686$ & 1 & 5 & 1 & 0 & 0 & 0 & 7 \\
\hline -Other & 700-709 & 2 & 2 & 1 & 1 & 0 & 0 & 6 \\
\hline $\begin{array}{l}\text { MUSCULOSKELETAL \& CONNECTIVE } \\
\text { TISSUE }\end{array}$ & 710-739 & 2 & 26 & 8 & 2 & 1 & 1 & 40 \\
\hline -Arthropathies & $710-719$ & 1 & 10 & 2 & 1 & 0 & 1 & 15 \\
\hline -Dorsopathies & $720-724$ & 0 & 9 & 3 & 0 & 0 & 0 & 12 \\
\hline -Rheumatism, Excluding Back & 725-729 & 1 & 7 & 2 & 1 & 1 & 0 & 12 \\
\hline -Other Dis \& Acquired Deformities & 730-739 & 0 & 0 & 1 & 0 & 0 & 0 & 1 \\
\hline CONGENITAL ANOMALIES & $740-759$ & 0 & 1 & 0 & 0 & 0 & 1 & 2 \\
\hline $\begin{array}{l}\text { SYMPTOMS, SIGNS, \& ILL-DEFINED } \\
\text { CONDITIONS }\end{array}$ & 780-799 & 1 & 7 & 1 & 0 & 1 & 0 & 10 \\
\hline -Symptoms & 780-789 & 1 & 7 & 1 & 0 & 1 & 0 & 10 \\
\hline INJURY \& POISONING & $800-999$ & 2 & 15 & 2 & 1 & 1 & 0 & 21 \\
\hline -Fracture - Upper Limb & \begin{tabular}{|c|}
$810-819$ \\
\end{tabular} & 0 & 2 & 0 & 0 & 0 & 0 & 2 \\
\hline -Fracture - Lower Limb & \begin{tabular}{|c|}
$820-829$ \\
\end{tabular} & 1 & 3 & 0 & 0 & 1 & 0 & 5 \\
\hline -Dislocation & $830-839$ & 0 & 1 & 1 & 0 & 0 & 0 & 2 \\
\hline
\end{tabular}

(Continued)

*Only those diagnostic categories and gender/job category combinations with at least one occurrence appear in this table. 


\section{Lawrence Livermore National Laboratory 2006}

Absence Data

Appendix I. Number of Diagnoses in Each Diagnostic Category by Gender and Job Category*

\begin{tabular}{|c|c|c|c|c|c|c|c|c|}
\hline & & \multicolumn{7}{|c|}{ Women } \\
\hline & & \multicolumn{6}{|c|}{ Job Category } & \multirow[b]{2}{*}{ TOTAL } \\
\hline & & Professional & $\begin{array}{l}\text { Administrative } \\
\text { Support }\end{array}$ & $\begin{array}{l}\text { Technical } \\
\text { Support }\end{array}$ & Service & $\begin{array}{c}\text { Security } \\
\text { and } \\
\text { Fire }\end{array}$ & Crafts & \\
\hline Diagnostic Category & $\begin{array}{l}\text { ICD-9-CM } \\
\text { Code }\end{array}$ & & & & & & & \\
\hline -Sprains \& Strains - Other & $\begin{array}{l}840-845 \\
848\end{array}$ & 0 & 5 & 0 & 1 & 0 & 0 & 6 \\
\hline -Superficial Injury & $910-919$ & 0 & 1 & 0 & 0 & 0 & 0 & 1 \\
\hline -Complications \& Unspecified Injuries & 958-959 & 1 & 0 & 0 & 0 & 0 & 0 & 1 \\
\hline -Complications of Surgical/Medical Care & 996-999 & 0 & 3 & 1 & 0 & 0 & 0 & 4 \\
\hline $\begin{array}{l}\text { HEALTH STATUS/HEALTH SERVICE } \\
\text { CONTACT }\end{array}$ & V01-V82 & 0 & 6 & 2 & 0 & 0 & 0 & 8 \\
\hline -Health Services Reproduction/Development & V20-V29 & 0 & 2 & 0 & 0 & 0 & 0 & 2 \\
\hline -Specific Procedures/Aftercare & V50-V59 & 0 & 3 & 2 & 0 & 0 & 0 & 5 \\
\hline -Examination \& Investigation & V70-V82 & 0 & 1 & 0 & 0 & 0 & 0 & 1 \\
\hline
\end{tabular}

\begin{tabular}{|c|c|c|c|c|c|c|c|}
\hline & \multicolumn{7}{|c|}{ Women } \\
\hline & \multicolumn{6}{|c|}{ Job Category } & \multirow[b]{2}{*}{ TOTAL } \\
\hline & Professional & $\begin{array}{l}\text { Administrative } \\
\text { Support }\end{array}$ & $\begin{array}{l}\text { Technical } \\
\text { Support }\end{array}$ & Service & $\begin{array}{c}\text { Security } \\
\text { and } \\
\text { Fire }\end{array}$ & Crafts & \\
\hline $\begin{array}{l}\text { Diagnostic } \\
\text { Category }\end{array}$ & \multirow[b]{2}{*}{25} & \multirow[b]{2}{*}{204} & \multirow[b]{2}{*}{32} & \multirow[b]{2}{*}{15} & \multirow[b]{2}{*}{7} & \multirow[b]{2}{*}{7} & \multirow[b]{2}{*}{290} \\
\hline Total & & & & & & & \\
\hline
\end{tabular}

*Only those diagnostic categories and gender/job category combinations with at least one occurrence appear in this table. 


\section{Lawrence Livermore National Laboratory 2006}

\section{Absence Data}

Appendix I. Number of Diagnoses in Each Diagnostic Category by Gender and Job Category*

\begin{tabular}{|c|c|c|c|c|c|c|c|c|}
\hline & & \multicolumn{7}{|c|}{ Men } \\
\hline & & \multicolumn{6}{|c|}{ Job Category } & \multirow[b]{2}{*}{ TOTAL } \\
\hline & & Professional & $\begin{array}{l}\text { Administrative } \\
\text { Support }\end{array}$ & $\begin{array}{l}\text { Technical } \\
\text { Support }\end{array}$ & Service & $\begin{array}{c}\text { Security } \\
\text { and } \\
\text { Fire }\end{array}$ & Crafts & \\
\hline Diagnostic Category & $\begin{array}{l}\text { ICD-9-CM } \\
\text { Code }\end{array}$ & & & & & & & \\
\hline $\begin{array}{l}\text { INFECTIOUS \& PARASITIC DISEASES } \\
\text { (DIS) }\end{array}$ & 001-139 & 9 & 4 & 4 & 1 & 0 & 2 & 20 \\
\hline -Intestinal Infectious Dis & 001-009 & 0 & 0 & 1 & 0 & 0 & 1 & 2 \\
\hline -Other Bacterial Dis & 030-041 & 2 & 1 & 0 & 0 & 0 & 0 & 3 \\
\hline -Viral Dis with Exanthem & 050-057 & 3 & 0 & 1 & 0 & 0 & 0 & 4 \\
\hline -Other Viral Dis \& Chlamydiae & 070-079 & 3 & 3 & 1 & 1 & 0 & 1 & 9 \\
\hline -Mycoses & 110-118 & 1 & 0 & 0 & 0 & 0 & 0 & 1 \\
\hline -Other Infections \& Parasitic Dis & $130-136$ & 0 & 0 & 1 & 0 & 0 & 0 & 1 \\
\hline MALIGNANT NEOPLASMS & $\begin{array}{l}140-208 \\
230-234\end{array}$ & 9 & 2 & 0 & 3 & 0 & 1 & 15 \\
\hline -Lip, Oral Cavity, Pharynx & $140-149$ & 1 & 0 & 0 & 0 & 0 & 0 & 1 \\
\hline -Digestive \& Peritoneal & $150-159$ & 0 & 0 & 0 & 0 & 0 & 1 & 1 \\
\hline -Bone, Connective Tissue, Skin & $\begin{array}{l}170-173 \\
176\end{array}$ & 1 & 0 & 0 & 1 & 0 & 0 & 2 \\
\hline -Genitourinary & 179-189 & 5 & 2 & 0 & 2 & 0 & 0 & 9 \\
\hline -Lymphatic \& Hematopoietic & $200-208$ & 2 & 0 & 0 & 0 & 0 & 0 & 2 \\
\hline BENIGN \& UNCERTAIN NEOPLASMS & $\begin{array}{l}210-229 \\
235-239\end{array}$ & 2 & 4 & 1 & 0 & 0 & 1 & 8 \\
\hline ENDOCRINE/METABOLIC/IMMUNITY & $240-279$ & 2 & 2 & 3 & 0 & 0 & 3 & 10 \\
\hline -Thyroid Gland Disorders & $240-246$ & 0 & 1 & 0 & 0 & 0 & 0 & 1 \\
\hline -Other Endocrine Gland Dis & $250-259$ & 0 & 1 & 0 & 0 & 0 & 0 & 1 \\
\hline -Other Metabolic \& Immunity Disorders & $270-279$ & 2 & 0 & 3 & 0 & 0 & 3 & 8 \\
\hline MENTAL DISORDERS & $290-319$ & 1 & 2 & 0 & 0 & 0 & 1 & 4 \\
\hline -Non-Psychotic Disorders & $\begin{array}{l}300-302 \\
306-316\end{array}$ & 1 & 2 & 0 & 0 & 0 & 1 & 4 \\
\hline $\begin{array}{l}\text { NERVOUS SYSTEM (NS) \& SENSE } \\
\text { ORGANS }\end{array}$ & $320-389$ & 7 & 8 & 9 & 1 & 2 & 1 & 28 \\
\hline -Inflammatory Dis of Central NS & $320-326$ & 0 & 0 & 1 & 0 & 0 & 0 & 1 \\
\hline -Hereditary/Degenerative Central NS Dis & 330-337 & 0 & 1 & 0 & 0 & 0 & 0 & 1 \\
\hline -Disorders of Peripheral NS & $350-359$ & 3 & 4 & 2 & 1 & 0 & 0 & 10 \\
\hline -Disorders of Eye & $360-379$ & 3 & 1 & 5 & 0 & 2 & 1 & 12 \\
\hline -Dis of Ear \& Mastoid & $380-389$ & 1 & 2 & 1 & 0 & 0 & 0 & 4 \\
\hline CIRCULATORY SYSTEM & $390-459$ & 11 & 7 & 8 & 1 & 0 & 4 & 31 \\
\hline -Hypertensive Dis & $401-405$ & 0 & 0 & 2 & 0 & 0 & 1 & 3 \\
\hline -Ischemic Heart Dis & $410-414$ & 4 & 2 & 1 & 0 & 0 & 0 & 7 \\
\hline -Other Heart Dis & $420-429$ & 1 & 2 & 1 & 0 & 0 & 1 & 5 \\
\hline -Cerebrovascular Dis & $430-438$ & 3 & 1 & 2 & 1 & 0 & 0 & 7 \\
\hline
\end{tabular}

(Continued)

*Only those diagnostic categories and gender/job category combinations with at least one occurrence appear in this table. 


\section{Lawrence Livermore National Laboratory 2006}

\section{Absence Data}

Appendix I. Number of Diagnoses in Each Diagnostic Category by Gender and Job Category*

\begin{tabular}{|c|c|c|c|c|c|c|c|c|}
\hline & & \multicolumn{7}{|c|}{ Men } \\
\hline & & \multicolumn{6}{|c|}{ Job Category } & \multirow[b]{2}{*}{ TOTAL } \\
\hline & & Professional & $\begin{array}{l}\text { Administrative } \\
\text { Support }\end{array}$ & $\begin{array}{l}\text { Technical } \\
\text { Support }\end{array}$ & Service & $\begin{array}{c}\text { Security } \\
\text { and } \\
\text { Fire }\end{array}$ & Crafts & \\
\hline Diagnostic Category & \begin{tabular}{|l|} 
ICD-9-CM \\
Code
\end{tabular} & & & & & & & \\
\hline -Dis of Arteries \& Capillaries & $440-448$ & 1 & 2 & 0 & 0 & 0 & 1 & 4 \\
\hline -Dis of Veins, Lymphatics, Other & $451-459$ & 2 & 0 & 2 & 0 & 0 & 1 & 5 \\
\hline RESPIRATORY SYSTEM & $460-519$ & 24 & 23 & 12 & 3 & 5 & 2 & 69 \\
\hline -Acute Respiratory Infections & $460-466$ & 10 & 8 & 8 & 1 & 2 & 2 & 31 \\
\hline -Other Dis Upper Respiratory Tract & $470-478$ & 5 & 3 & 1 & 1 & 0 & 0 & 10 \\
\hline -Pneumonia \& Influenza & $480-487$ & 9 & 8 & 1 & 1 & 3 & 0 & 22 \\
\hline -Chronic Obstructive Dis & $490-496$ & 0 & 4 & 1 & 0 & 0 & 0 & 5 \\
\hline -Other Respiratory Dis & $510-519$ & 0 & 0 & 1 & 0 & 0 & 0 & 1 \\
\hline DIGESTIVE SYSTEM & $520-579$ & 21 & 13 & 6 & 2 & 1 & 4 & 47 \\
\hline -Oral Cavity, Saliva Glands, Jaw & $520-529$ & 1 & 0 & 1 & 0 & 1 & 0 & 3 \\
\hline -Esophagus, Stomach, Duodenum & $530-537$ & 0 & 1 & 0 & 0 & 0 & 0 & 1 \\
\hline -Appendicitis & $540-543$ & 1 & 0 & 1 & 1 & 0 & 1 & 4 \\
\hline -Hernia & $550-553$ & 4 & 6 & 1 & 0 & 0 & 2 & 13 \\
\hline -Enteritis, Colitis & $555-558$ & 3 & 1 & 0 & 0 & 0 & 0 & 4 \\
\hline -Other Intestinal Dis & $560-569$ & 6 & 1 & 2 & 0 & 0 & 1 & 10 \\
\hline -Other Digestive Dis & $570-579$ & 6 & 4 & 1 & 1 & 0 & 0 & 12 \\
\hline GENITOURINARY SYSTEM & $580-629$ & 8 & 3 & 0 & 2 & 1 & 1 & 15 \\
\hline -Other Urinary Dis & $590-599$ & 7 & 3 & 0 & 1 & 1 & 1 & 13 \\
\hline -Male Genital Organ Dis & $600-608$ & 1 & 0 & 0 & 1 & 0 & 0 & 2 \\
\hline SKIN \& SUBCUTANEOUS TISSUE & $680-709$ & 1 & 5 & 2 & 0 & 1 & 2 & 11 \\
\hline -Infections & $680-686$ & 1 & 1 & 1 & 0 & 0 & 1 & 4 \\
\hline -Other Inflammatory Conditions & $690-698$ & 0 & 0 & 0 & 0 & 1 & 0 & 1 \\
\hline -Other & 700-709 & 0 & 4 & 1 & 0 & 0 & 1 & 6 \\
\hline $\begin{array}{l}\text { MUSCULOSKELETAL \& CONNECTIVE } \\
\text { TISSUE }\end{array}$ & $710-739$ & 14 & 34 & 22 & 7 & 1 & 6 & 84 \\
\hline -Arthropathies & $710-719$ & 5 & 13 & 11 & 0 & 0 & 1 & 30 \\
\hline -Dorsopathies & $720-724$ & 7 & 15 & 8 & 6 & 1 & 3 & 40 \\
\hline -Rheumatism, Excluding Back & 725-729 & 1 & 6 & 2 & 1 & 0 & 2 & 12 \\
\hline -Other Dis \& Acquired Deformities & $730-739$ & 1 & 0 & 1 & 0 & 0 & 0 & 2 \\
\hline CONGENITAL ANOMALIES & $740-759$ & 0 & 1 & 0 & 0 & 0 & 0 & 1 \\
\hline $\begin{array}{l}\text { SYMPTOMS, SIGNS, \& ILL-DEFINED } \\
\text { CONDITIONS }\end{array}$ & $780-799$ & 6 & 5 & 5 & 4 & 4 & 1 & 25 \\
\hline -Symptoms & $780-789$ & 5 & 5 & 5 & 4 & 4 & 1 & 24 \\
\hline -Non-Specific Abnormal Findings & $790-796$ & 1 & 0 & 0 & 0 & 0 & 0 & 1 \\
\hline INJURY \& POISONING & \begin{tabular}{|l|}
$800-999$ \\
\end{tabular} & 24 & 32 & 12 & 3 & 7 & 10 & 88 \\
\hline
\end{tabular}

(Continued)

*Only those diagnostic categories and gender/job category combinations with at least one occurrence appear in this table. 


\section{Lawrence Livermore National Laboratory 2006}

Absence Data

Appendix I. Number of Diagnoses in Each Diagnostic Category by Gender and Job Category*

\begin{tabular}{|c|c|c|c|c|c|c|c|c|}
\hline & & \multicolumn{7}{|c|}{ Men } \\
\hline & & \multicolumn{6}{|c|}{ Job Category } & \multirow[b]{2}{*}{ TOTAL } \\
\hline & & Professional & $\begin{array}{c}\text { Administrative } \\
\text { Support }\end{array}$ & $\begin{array}{l}\text { Technical } \\
\text { Support }\end{array}$ & Service & $\begin{array}{c}\text { Security } \\
\text { and } \\
\text { Fire }\end{array}$ & Crafts & \\
\hline Diagnostic Category & \begin{tabular}{|l|} 
ICD-9-CM \\
Code
\end{tabular} & & & & & & & \\
\hline -Fracture - Skull & \begin{tabular}{|l|}
$800-804$ \\
\end{tabular} & 0 & 1 & 1 & 0 & 0 & 0 & 2 \\
\hline -Fracture - Neck, Trunk & \begin{tabular}{|l|}
$805-809$ \\
\end{tabular} & 0 & 2 & 1 & 0 & 0 & 0 & 3 \\
\hline -Fracture - Upper Limb & $810-819$ & 2 & 2 & 0 & 0 & 0 & 1 & 5 \\
\hline -Fracture - Lower Limb & $820-829$ & 5 & 4 & 1 & 0 & 1 & 1 & 12 \\
\hline -Dislocation & \begin{tabular}{|l|}
$830-839$ \\
\end{tabular} & 5 & 6 & 1 & 1 & 0 & 2 & 15 \\
\hline -Sprains \& Strains - Back & 846-847 & 2 & 2 & 1 & 1 & 2 & 2 & 10 \\
\hline -Sprains \& Strains - Other & $\begin{array}{l}840-845 \\
848\end{array}$ & 5 & 5 & 4 & 0 & 3 & 3 & 20 \\
\hline -Internal Injury - Thorax, Abdomen, Pelvis & $860-869$ & 0 & 0 & 1 & 0 & 0 & 0 & 1 \\
\hline -Open Wound - Head, Neck, Trunk & $870-879$ & 1 & 1 & 0 & 0 & 0 & 0 & 2 \\
\hline -Open Wound - Upper Limb & $880-887$ & 1 & 1 & 0 & 1 & 0 & 0 & 3 \\
\hline -Late Effects of Accident & $905-909$ & 0 & 1 & 0 & 0 & 0 & 0 & 1 \\
\hline -Contusion & $920-924$ & 2 & 1 & 0 & 0 & 0 & 0 & 3 \\
\hline -Burns & 940-949 & 0 & 0 & 0 & 0 & 1 & 0 & 1 \\
\hline -Complications \& Unspecified Injuries & 958-959 & 1 & 5 & 1 & 0 & 0 & 1 & 8 \\
\hline -Unspecified Effects - External Causes & $990-995$ & 0 & 1 & 0 & 0 & 0 & 0 & 1 \\
\hline -Complications of Surgical/Medical Care & 996-999 & 0 & 0 & 1 & 0 & 0 & 0 & 1 \\
\hline $\begin{array}{l}\text { HEALTH STATUS/HEALTH SERVICE } \\
\text { CONTACT }\end{array}$ & V01-V82 & 1 & 4 & 1 & 0 & 1 & 0 & 7 \\
\hline -Personal \& Family History & V10-V19 & 1 & 0 & 0 & 0 & 0 & 0 & 1 \\
\hline -Health Services Reproduction/Development & V20-V29 & 0 & 2 & 0 & 0 & 1 & 0 & 3 \\
\hline -Specific Procedures/Aftercare & V50-V59 & 0 & 1 & 1 & 0 & 0 & 0 & 2 \\
\hline -Other Circumstances & V60-V69 & 0 & 1 & 0 & 0 & 0 & 0 & 1 \\
\hline
\end{tabular}

\begin{tabular}{|c|c|c|c|c|c|c|c|}
\hline & \multicolumn{7}{|c|}{ Men } \\
\hline & \multicolumn{6}{|c|}{ Job Category } & \multirow[b]{2}{*}{ TOTAL } \\
\hline & Professional & $\begin{array}{l}\text { Administrative } \\
\text { Support }\end{array}$ & $\begin{array}{c}\text { Technical } \\
\text { Support }\end{array}$ & Service & $\begin{array}{c}\text { Security } \\
\text { and } \\
\text { Fire }\end{array}$ & Crafts & \\
\hline $\begin{array}{l}\text { Diagnostic } \\
\text { Category }\end{array}$ & & & & & & & \\
\hline Total & 140 & 149 & 85 & 27 & 23 & 39 & 463 \\
\hline
\end{tabular}

*Only those diagnostic categories and gender/job category combinations with at least one occurrence appear in this table. 


\section{Lawrence Livermore National Laboratory 2006}

\section{Absence Data}

Appendix J. Total Number of Calendar Days Absent in Each Diagnostic Category by Gender and Job Category*

\begin{tabular}{|c|c|c|c|c|c|c|c|c|}
\hline & & \multicolumn{7}{|c|}{ Women } \\
\hline & & \multicolumn{6}{|c|}{ Job Category } & \multirow[b]{2}{*}{ TOTAL } \\
\hline & & Professional & $\begin{array}{c}\text { Administrative } \\
\text { Support }\end{array}$ & $\begin{array}{l}\text { Technical } \\
\text { Support }\end{array}$ & Service & $\begin{array}{c}\text { Security } \\
\text { and } \\
\text { Fire }\end{array}$ & Crafts & \\
\hline Diagnostic Category & $\begin{array}{l}\text { ICD-9-CM } \\
\text { Code }\end{array}$ & & & & & & & \\
\hline $\begin{array}{l}\text { INFECTIOUS \& PARASITIC DISEASES } \\
\text { (DIS) }\end{array}$ & 001-139 & 10 & 211 & 69 & 11 & 13 & 0 & 314 \\
\hline MALIGNANT NEOPLASMS & \begin{tabular}{|l}
$140-208$ \\
$230-234$
\end{tabular} & 50 & 76 & 111 & 0 & 0 & 0 & 237 \\
\hline BENIGN \& UNCERTAIN NEOPLASMS & $\begin{array}{l}210-229 \\
235-239\end{array}$ & 0 & 319 & 32 & 15 & 0 & 137 & 503 \\
\hline ENDOCRINE/METABOLIC/IMMUNITY & $240-279$ & 1 & 450 & 65 & 0 & 0 & 0 & 516 \\
\hline MENTAL DISORDERS & $290-319$ & 10 & 484 & 0 & 0 & 0 & 0 & 494 \\
\hline $\begin{array}{l}\text { NERVOUS SYSTEM (NS) \& SENSE } \\
\text { ORGANS }\end{array}$ & 320-389 & 0 & 185 & 0 & 0 & 0 & 76 & 261 \\
\hline CIRCULATORY SYSTEM & $390-459$ & 0 & 140 & 12 & 0 & 0 & 0 & 152 \\
\hline RESPIRATORY SYSTEM & $460-519$ & 53 & 217 & 33 & 72 & 7 & 41 & 423 \\
\hline DIGESTIVE SYSTEM & $520-579$ & 37 & 573 & 112 & 0 & 0 & 0 & 722 \\
\hline GENITOURINARY SYSTEM & $580-629$ & 18 & 821 & 45 & 44 & 7 & 0 & 935 \\
\hline PREGNANCY \& CHILDBIRTH & 630-677 & 0 & 0 & 5 & 0 & 0 & 0 & 5 \\
\hline SKIN \& SUBCUTANEOUS TISSUE & 680-709 & 109 & 183 & 5 & 62 & 0 & 0 & 359 \\
\hline $\begin{array}{l}\text { MUSCULOSKELETAL \& CONNECTIVE } \\
\text { TISSUE }\end{array}$ & 710-739 & 33 & 1,190 & 186 & 200 & 170 & 26 & 1,805 \\
\hline CONGENITAL ANOMALIES & 740-759 & 0 & 12 & 0 & 0 & 0 & 26 & 38 \\
\hline $\begin{array}{l}\text { SYMPTOMS, SIGNS, \& ILL-DEFINED } \\
\text { CONDITIONS }\end{array}$ & 780-799 & 10 & 88 & 4 & 0 & 4 & 0 & 106 \\
\hline INJURY \& POISONING & $800-999$ & 11 & 640 & 20 & 4 & 9 & 0 & 684 \\
\hline
\end{tabular}

\begin{tabular}{|c|c|c|c|c|c|c|c|c|}
\hline & & \multicolumn{7}{|c|}{ Men } \\
\hline & & \multicolumn{6}{|c|}{ Job Category } & \multirow[b]{2}{*}{ TOTAL } \\
\hline & & Professional & $\begin{array}{c}\text { Administrative } \\
\text { Support }\end{array}$ & $\begin{array}{c}\text { Technical } \\
\text { Support }\end{array}$ & Service & \begin{tabular}{|c|}
$\begin{array}{c}\text { Security } \\
\text { and } \\
\text { Fire }\end{array}$ \\
\end{tabular} & Crafts & \\
\hline Diagnostic Category & $\begin{array}{l}\text { ICD-9-CM } \\
\text { Code }\end{array}$ & & & & & & & \\
\hline $\begin{array}{l}\text { INFECTIOUS \& PARASITIC DISEASES } \\
\text { (DIS) }\end{array}$ & 001-139 & 157 & 47 & 27 & 10 & 0 & 12 & 253 \\
\hline MALIGNANT NEOPLASMS & \begin{tabular}{|l|}
$140-208$ \\
$230-234$
\end{tabular} & 413 & 56 & 0 & 83 & 0 & 292 & 844 \\
\hline BENIGN \& UNCERTAIN NEOPLASMS & \begin{tabular}{|l}
$210-229$ \\
$235-239$
\end{tabular} & 110 & 81 & 6 & 0 & 0 & 43 & 240 \\
\hline ENDOCRINE/METABOLIC/IMMUNITY & 240-279 & 18 & 16 & 45 & 0 & 0 & 75 & 154 \\
\hline MENTAL DISORDERS & $290-319$ & 13 & 117 & 0 & 0 & 0 & 5 & 135 \\
\hline
\end{tabular}

(Continued) 


\section{Lawrence Livermore National Laboratory 2006}

Absence Data

Appendix J. Total Number of Calendar Days Absent in Each Diagnostic Category by Gender and Job Category*

\begin{tabular}{|c|c|c|c|c|c|c|c|c|}
\hline & & \multicolumn{7}{|c|}{ Men } \\
\hline & & \multicolumn{6}{|c|}{ Job Category } & \multirow[b]{2}{*}{ TOTAL } \\
\hline & & Professional & $\begin{array}{l}\text { Administrative } \\
\text { Support }\end{array}$ & $\begin{array}{l}\text { Technical } \\
\text { Support }\end{array}$ & Service & $\begin{array}{c}\text { Security } \\
\text { and } \\
\text { Fire }\end{array}$ & Crafts & \\
\hline Diagnostic Category & $\begin{array}{l}\text { ICD-9-CM } \\
\text { Code }\end{array}$ & & & & & & & \\
\hline $\begin{array}{l}\text { NERVOUS SYSTEM (NS) \& SENSE } \\
\text { ORGANS }\end{array}$ & $320-389$ & 76 & 229 & 335 & 5 & 11 & 18 & 674 \\
\hline CIRCULATORY SYSTEM & $390-459$ & 222 & 177 & 254 & 97 & 0 & 121 & 871 \\
\hline RESPIRATORY SYSTEM & $460-519$ & 233 & 236 & 105 & 35 & 24 & 18 & 651 \\
\hline DIGESTIVE SYSTEM & $520-579$ & 351 & 143 & 114 & 40 & 46 & 70 & 764 \\
\hline GENITOURINARY SYSTEM & $580-629$ & 53 & 53 & 0 & 17 & 14 & 5 & 142 \\
\hline SKIN \& SUBCUTANEOUS TISSUE & $680-709$ & 13 & 96 & 8 & 0 & 11 & 45 & 173 \\
\hline $\begin{array}{l}\text { MUSCULOSKELETAL \& CONNECTIVE } \\
\text { TISSUE }\end{array}$ & $710-739$ & 265 & 969 & 646 & 148 & 11 & 112 & 2,151 \\
\hline CONGENITAL ANOMALIES & $740-759$ & 0 & 1 & 0 & 0 & 0 & 0 & 1 \\
\hline $\begin{array}{l}\text { SYMPTOMS, SIGNS, \& ILL-DEFINED } \\
\text { CONDITIONS }\end{array}$ & 780-799 & 106 & 33 & 188 & 69 & 34 & 8 & 438 \\
\hline INJURY \& POISONING & 800-999 & 242 & 621 & 329 & 121 & 166 & 418 & 1,897 \\
\hline
\end{tabular}

\footnotetext{
*Absences with >1 ICD-9-CM code in the same diagnostic category were counted only once. Only those diagnostic categories and gender/job category combinations with at least one occurrence appear in this table.
} 


\section{Lawrence Livermore National Laboratory 2006}

\section{Absence Data}

Appendix K. Age-Adjusted IIlness and Injury Rates by Diagnostic Category

Part 1. Men

\begin{tabular}{|c|c|c|c|c|c|}
\hline Category of Diagnoses & ICD-9-CM Code & $\begin{array}{l}\text { Number of } \\
\text { Diagnoses }\end{array}$ & $\begin{array}{c}\text { Age-Adjusted } \\
\text { Rate per } 1000 *\end{array}$ & $\begin{array}{c}\text { Lower } 95 \% \\
\text { Confidence } \\
\text { Limit per } 1000\end{array}$ & $\begin{array}{c}\text { Upper 95\% } \\
\text { Confidence } \\
\text { Limit per } 1000\end{array}$ \\
\hline Infections/Parasites & $001-139$ & 20 & 2.4 & 1.5 & 3.9 \\
\hline Cancer & $140-208,230-234$ & 15 & 1.5 & 0.9 & 2.6 \\
\hline -Lip, Oral Cavity, Pharynx & $140-149$ & 1 & 0.1 & 0.0 & 0.9 \\
\hline -Digestive Organs & $150-159$ & 1 & 0.1 & 0.0 & 0.6 \\
\hline -Respiratory System & $160-165$ & 0 & 0 & 0 & 0 \\
\hline -Bone, Connective Tissue, Skin & $170-173,176$ & 2 & 0.3 & 0.1 & 1.0 \\
\hline -Breast & $174-175$ & 0 & 0 & 0 & $\overline{0}$ \\
\hline -Genitourinary & $179-189$ & 9 & 0.9 & 0.4 & 1.7 \\
\hline -Other \& Unspecified Sites & $190,193-199$ & 0 & 0 & 0 & $\overline{0}$ \\
\hline -Nervous System & 191-192 & 0 & 0 & 0 & 0 \\
\hline -Leukemia, Lymphoma & $200-208$ & 2 & 0.2 & 0.0 & 0.8 \\
\hline -Carcinoma in situ & $230-234$ & 0 & 0 & 0 & 0 \\
\hline Benign Growths & $210-229,235-239$ & 8 & 0.7 & 0.4 & 1.5 \\
\hline Endocrine/Metabolic & $240-279$ & 10 & 1.5 & 0.7 & 3.2 \\
\hline -Other Endocrine Glands & $250-259$ & 1 & 0.1 & 0.0 & 0.9 \\
\hline Blood & $280-289$ & 0 & 0 & 0 & 0 \\
\hline Mental & $290-319$ & 4 & 0.7 & 0.2 & 2.6 \\
\hline -Non-Psychotic Conditions & $300-302,306-316$ & 4 & 0.7 & 0.2 & 2.6 \\
\hline Nervous System & $320-389$ & 28 & 3.4 & 2.2 & 5.3 \\
\hline -Disorders of Peripheral NS & $350-359$ & 10 & 1.1 & 0.6 & 2.2 \\
\hline Heart/Circulatory & $390-459$ & 31 & 3.2 & 2.2 & 4.5 \\
\hline -Hypertensive Disease & $401-405$ & 3 & 0.3 & 0.1 & 1.1 \\
\hline -Ischemic Heart Disease & $410-414$ & 7 & 0.6 & 0.3 & 1.3 \\
\hline Respiratory & $460-519$ & 69 & 8.8 & 6.7 & 11.5 \\
\hline -Chronic Obstructive Diseases & $490-496$ & 5 & 0.5 & 0.2 & 1.1 \\
\hline -Lung Disease from External Agents & $500-508$ & 0 & 0 & 0 & 0 \\
\hline Digestive & $520-579$ & 47 & 6.1 & 4.3 & 8.7 \\
\hline -Hernias & $550-553$ & 13 & 1.6 & 0.8 & 3.2 \\
\hline Genitourinary & $580-629$ & 15 & 1.8 & 1.0 & 3.0 \\
\hline -Nephritis, Nephrosis & $580-589$ & 0 & 0 & 0 & 0 \\
\hline Miscarriage $* *^{* *}$ & $630-677$ & 0 & 0 & 0 & 0 \\
\hline Skin & $680-709$ & 11 & 1.5 & 0.8 & 2.8 \\
\hline Musculoskeletal & $710-739$ & 84 & 10.3 & 8.1 & 13.1 \\
\hline -Arthropathies & $710-719$ & 30 & 3.5 & 2.3 & 5.3 \\
\hline -Dorsopathies & $720-724$ & 40 & 5.4 & 3.8 & 7.6 \\
\hline -Rheumatism, Excluding Back & $725-729$ & 12 & 1.2 & 0.7 & 2.1 \\
\hline Congenital Anomalies & $740-759$ & 1 & 0.1 & 0.0 & $\overline{0.6}$ \\
\hline
\end{tabular}

*Standardized to age distribution of 2000 U.S. population.

**Only women aged 18-45 were included in the calculation of the rates for these diagnostic categories. 


\section{Lawrence Livermore National Laboratory 2006}

Absence Data

Appendix K. Age-Adjusted Illness and Injury Rates by Diagnostic Category

\begin{tabular}{|c|c|c|c|c|c|}
\hline Category of Diagnoses & ICD-9-CM Code & $\begin{array}{l}\text { Number of } \\
\text { Diagnoses }\end{array}$ & $\begin{array}{l}\text { Age-Adjusted } \\
\text { Rate per } 1000 *\end{array}$ & $\begin{array}{c}\text { Lower } 95 \% \\
\text { Confidence } \\
\text { Limit per } 1000\end{array}$ & $\begin{array}{c}\text { Upper } 95 \% \\
\text { Confidence } \\
\text { Limit per } 1000\end{array}$ \\
\hline Unspecified Symptoms & $780-799$ & 25 & 3.3 & 2.0 & 5.4 \\
\hline Injury & $800-999$ & 88 & 10.9 & 8.6 & 13.9 \\
\hline -Fractures - Skull, Neck, Trunk & $800-809$ & 5 & 0.6 & 0.2 & 1.6 \\
\hline -Fractures - Upper Limb & $810-819$ & 5 & 0.6 & 0.3 & 1.5 \\
\hline -Fractures - Lower Limb & $820-829$ & 12 & 1.5 & 0.8 & 2.9 \\
\hline -Dislocations & $830-839$ & 15 & 1.8 & 1.0 & 3.1 \\
\hline -Back Sprains \& Strains & $846-847$ & 10 & 1.5 & 0.7 & 3.2 \\
\hline -Other Sprains \& Strains & $840-845,848$ & 20 & 2.8 & 1.6 & 4.6 \\
\hline -Open Wounds & $870-887,890-897$ & 5 & 0.5 & 0.2 & 1.1 \\
\hline -Burns & $940-949$ & 1 & 0.1 & 0.0 & $\overline{0.6}$ \\
\hline -Adverse Reactions to Nonmedical Substances & $980-989$ & 0 & 0 & 0 & 0 \\
\hline -Adverse Reactions to External Causes & $990-995$ & 1 & 0.1 & 0.0 & 0.6 \\
\hline Health Status/Health Service Contact & V01-V82 & 0 & 0 & 0 & 0 \\
\hline Total & & 456 & 56.3 & 50.6 & 62.5 \\
\hline
\end{tabular}

Part 2. Women

\begin{tabular}{|c|c|c|c|c|c|}
\hline Category of Diagnoses & ICD-9-CM Code & $\begin{array}{l}\text { Number of } \\
\text { Diagnoses }\end{array}$ & $\begin{array}{l}\text { Age-Adjusted } \\
\text { Rate per } 1000 *\end{array}$ & $\begin{array}{c}\text { Lower } 95 \% \\
\text { Confidence } \\
\text { Limit per } 1000\end{array}$ & $\begin{array}{c}\text { Upper } 95 \% \\
\text { Confidence } \\
\text { Limit per } 1000\end{array}$ \\
\hline Infections/Parasites & 001-139 & 13 & 4.1 & 2.2 & 7.8 \\
\hline Cancer & $140-208,230-234$ & 5 & 1.1 & 0.5 & 2.6 \\
\hline -Lip, Oral Cavity, Pharynx & $140-149$ & 0 & 0 & 0 & 0 \\
\hline -Digestive Organs & $150-159$ & 0 & 0 & 0 & 0 \\
\hline -Respiratory System & $160-165$ & 0 & 0 & 0 & 0 \\
\hline -Bone, Connective Tissue, Skin & $170-173,176$ & 0 & 0 & 0 & 0 \\
\hline -Breast & $174-175$ & 4 & 0.8 & 0.3 & 2.3 \\
\hline -Genitourinary & $179-189$ & 1 & 0.2 & 0.0 & 1.8 \\
\hline -Other \& Unspecified Sites & $190,193-199$ & 0 & 0 & 0 & 0 \\
\hline -Nervous System & 191-192 & 0 & 0 & 0 & 0 \\
\hline -Leukemia, Lymphoma & $200-208$ & 0 & 0 & 0 & 0 \\
\hline -Carcinoma in situ & $230-234$ & 0 & 0 & 0 & 0 \\
\hline Benign Growths & $210-229,235-239$ & 10 & 3.1 & 1.5 & 6.3 \\
\hline Endocrine/Metabolic & $240-279$ & 17 & 4.0 & 2.4 & 6.6 \\
\hline -Other Endocrine Glands & $250-259$ & 1 & 0.2 & 0.0 & 1.4 \\
\hline Blood & $280-289$ & 0 & 0 & 0 & 0 \\
\hline Mental & $290-319$ & 7 & 3.2 & 1.4 & 7.4 \\
\hline -Non-Psychotic Conditions & $300-302,306-316$ & 6 & 2.4 & 1.0 & 6.2 \\
\hline Nervous System & $320-389$ & 15 & 4.1 & 2.3 & 7.2 \\
\hline
\end{tabular}

*Standardized to age distribution of 2000 U.S. population.

**Only women aged 18-45 were included in the calculation of the rates for these diagnostic categories. 


\section{Lawrence Livermore National Laboratory 2006}

Absence Data

Appendix K. Age-Adjusted Illness and Injury Rates by Diagnostic Category

\begin{tabular}{|c|c|c|c|c|c|}
\hline Category of Diagnoses & ICD-9-CM Code & $\begin{array}{l}\text { Number of } \\
\text { Diagnoses }\end{array}$ & $\begin{array}{l}\text { Age-Adjusted } \\
\text { Rate per } 1000 *\end{array}$ & $\begin{array}{c}\text { Lower } 95 \% \\
\text { Confidence } \\
\text { Limit per } 1000\end{array}$ & $\begin{array}{c}\text { Upper } 95 \% \\
\text { Confidence } \\
\text { Limit per } 1000\end{array}$ \\
\hline -Disorders of Peripheral NS & $350-359$ & 6 & 1.3 & 0.6 & 3.0 \\
\hline Heart/Circulatory & $390-459$ & 9 & 2.0 & 1.0 & 3.8 \\
\hline -Hypertensive Disease & $401-405$ & 0 & 0 & 0 & 0 \\
\hline -Ischemic Heart Disease & $410-414$ & 1 & 0.2 & 0.0 & 1.4 \\
\hline Respiratory & $460-519$ & 47 & 16.6 & 12.0 & 23.0 \\
\hline -Chronic Obstructive Diseases & $490-496$ & 7 & 2.2 & 1.0 & 5.0 \\
\hline -Lung Disease from External Agents & $500-508$ & 0 & 0 & 0 & 0 \\
\hline Digestive & $520-579$ & 30 & 9.3 & 6.3 & 13.8 \\
\hline -Hernias & $550-553$ & 4 & 0.8 & 0.3 & 2.3 \\
\hline Genitourinary & $580-629$ & 42 & 11.3 & 8.1 & 15.6 \\
\hline -Nephritis, Nephrosis & $580-589$ & 4 & 1.4 & 0.5 & 4.0 \\
\hline Miscarriage** & $630-677$ & 1 & 0.5 & 0.1 & 3.5 \\
\hline Skin & $680-709$ & 13 & 3.3 & 1.8 & 5.9 \\
\hline Musculoskeletal & $710-739$ & 40 & 12.9 & 9.2 & 18.1 \\
\hline -Arthropathies & $710-719$ & 15 & 5.4 & 3.1 & 9.5 \\
\hline -Dorsopathies & $720-724$ & 12 & 4.1 & 2.2 & 7.4 \\
\hline -Rheumatism, Excluding Back & $725-729$ & 12 & 3.2 & 1.7 & 5.9 \\
\hline Congenital Anomalies & $740-759$ & 2 & 0.4 & 0.1 & 1.6 \\
\hline Unspecified Symptoms & $780-799$ & 10 & 2.2 & 1.2 & 4.1 \\
\hline Injury & $800-999$ & 21 & 6.8 & 4.2 & 10.9 \\
\hline -Fractures - Skull, Neck, Trunk & $800-809$ & 0 & 0 & 0 & 0 \\
\hline -Fractures - Upper Limb & $810-819$ & 2 & 1.1 & 0.3 & 4.3 \\
\hline -Fractures - Lower Limb & $820-829$ & 5 & 1.1 & 0.5 & 2.6 \\
\hline -Dislocations & $830-839$ & 2 & 0.8 & 0.2 & 3.4 \\
\hline -Back Sprains \& Strains & $846-847$ & 0 & 0 & 0 & 0 \\
\hline -Other Sprains \& Strains & $840-845,848$ & 6 & 2.2 & 0.9 & 5.4 \\
\hline -Open Wounds & $870-887,890-897$ & 0 & 0 & 0 & 0 \\
\hline -Burns & $940-949$ & 0 & 0 & 0 & 0 \\
\hline -Adverse Reactions to Nonmedical Substances & $980-989$ & 0 & 0 & 0 & 0 \\
\hline -Adverse Reactions to External Causes & $990-995$ & 0 & 0 & 0 & 0 \\
\hline Health Status/Health Service Contact & V01-V82 & 0 & 0 & 0 & 0 \\
\hline Total & & 282 & 84.8 & 74.4 & 96.7 \\
\hline
\end{tabular}

*Standardized to age distribution of 2000 U.S. population.

**Only women aged 18-45 were included in the calculation of the rates for these diagnostic categories. 


\section{Lawrence Livermore National Laboratory 2006}

\section{Absence Data}

Appendix K. Age-Adjusted IIlness and Injury Rates by Diagnostic Category

Part 3. Men and Women

\begin{tabular}{|c|c|c|c|c|c|}
\hline Category of Diagnoses & ICD-9-CM Code & $\begin{array}{c}\text { Number of } \\
\text { Diagnoses }\end{array}$ & $\begin{array}{l}\text { Age-Adjusted } \\
\text { Rate per } 1000 *\end{array}$ & \begin{tabular}{|c|} 
Lower $95 \%$ \\
Confidence \\
Limit per 1000
\end{tabular} & $\begin{array}{c}\text { Upper } 95 \% \\
\text { Confidence } \\
\text { Limit per } 1000\end{array}$ \\
\hline Infections/Parasites & $001-139$ & 33 & 3.1 & 2.1 & $\overline{4.6}$ \\
\hline Cancer & $140-208,230-234$ & 20 & 1.5 & 0.9 & 2.3 \\
\hline -Lip, Oral Cavity, Pharynx & $140-149$ & 1 & 0.1 & 0.0 & 0.6 \\
\hline -Digestive Organs & $150-159$ & 1 & 0.1 & 0.0 & 0.4 \\
\hline -Respiratory System & $160-165$ & 0 & 0 & 0 & 0 \\
\hline -Bone, Connective Tissue, Skin & $170-173,176$ & 2 & 0.2 & 0.0 & 0.8 \\
\hline -Breast & $174-175$ & 4 & 0.3 & 0.1 & 0.7 \\
\hline -Genitourinary & $179-189$ & 10 & 0.7 & 0.4 & 1.4 \\
\hline -Other \& Unspecified Sites & $190,193-199$ & 0 & 0 & 0 & 0 \\
\hline -Nervous System & $191-192$ & 0 & 0 & 0 & 0 \\
\hline -Leukemia, Lymphoma & $200-208$ & 2 & 0.2 & 0.0 & 0.7 \\
\hline -Carcinoma in situ & $230-234$ & 0 & 0 & 0 & 0 \\
\hline Benign Growths & $210-229,235-239$ & 18 & 1.5 & 0.9 & 2.6 \\
\hline Endocrine/Metabolic & $240-279$ & 27 & 2.2 & 1.5 & 3.4 \\
\hline -Other Endocrine Glands & $250-259$ & 2 & 0.2 & 0.0 & 0.7 \\
\hline Blood & $280-289$ & 0 & 0 & 0 & 0 \\
\hline Mental & $290-319$ & 11 & 1.6 & 0.8 & 3.3 \\
\hline -Non-Psychotic Conditions & $300-302,306-316$ & 10 & 1.3 & 0.6 & 2.8 \\
\hline Nervous System & $320-389$ & 43 & 3.7 & 2.6 & 5.3 \\
\hline -Disorders of Peripheral NS & $350-359$ & 16 & 1.2 & 0.7 & 2.0 \\
\hline Heart/Circulatory & $390-459$ & 40 & 3.0 & 2.1 & 4.1 \\
\hline -Hypertensive Disease & $401-405$ & 3 & 0.2 & 0.1 & 0.7 \\
\hline -Ischemic Heart Disease & $410-414$ & 8 & 0.5 & 0.2 & 1.0 \\
\hline Respiratory & $460-519$ & 116 & 11.1 & 9.0 & 13.7 \\
\hline -Chronic Obstructive Diseases & $490-496$ & 12 & 0.9 & 0.5 & 1.6 \\
\hline -Lung Disease from External Agents & $500-508$ & 0 & 0 & 0 & 0 \\
\hline Digestive & $520-579$ & 77 & 7.2 & 5.6 & 9.3 \\
\hline -Hernias & $550-553$ & 17 & 1.4 & 0.8 & 2.5 \\
\hline Genitourinary & $580-629$ & 57 & 4.8 & 3.6 & 6.3 \\
\hline -Nephritis, Nephrosis & $580-589$ & 4 & 0.4 & 0.1 & 1.3 \\
\hline Miscarriage $^{* * *}$ & $630-677$ & 1 & 0.2 & 0.0 & 1.1 \\
\hline Skin & $680-709$ & 24 & 2.0 & 1.3 & 3.1 \\
\hline Musculoskeletal & $710-739$ & 124 & 11.1 & 9.1 & 13.5 \\
\hline -Arthropathies & $710-719$ & 45 & 4.0 & 2.9 & 5.6 \\
\hline -Dorsopathies & $720-724$ & 52 & 5.1 & 3.8 & 6.9 \\
\hline -Rheumatism, Excluding Back & $725-729$ & 24 & 1.8 & 1.2 & 2.7 \\
\hline Congenital Anomalies & $740-759$ & 3 & 0.2 & 0.1 & 0.5 \\
\hline
\end{tabular}

*Standardized to age distribution of 2000 U.S. population.

**Only women aged 18-45 were included in the calculation of the rates for these diagnostic categories. 
Lawrence Livermore National Laboratory 2006

Absence Data

Appendix K. Age-Adjusted Illness and Injury Rates by Diagnostic Category

\begin{tabular}{|c|c|c|c|c|c|}
\hline Category of Diagnoses & ICD-9-CM Code & $\begin{array}{l}\text { Number of } \\
\text { Diagnoses }\end{array}$ & $\begin{array}{l}\text { Age-Adjusted } \\
\text { Rate per } 1000 *\end{array}$ & $\begin{array}{c}\text { Lower } 95 \% \\
\text { Confidence } \\
\text { Limit per } 1000\end{array}$ & $\begin{array}{c}\text { Upper } 95 \% \\
\text { Confidence } \\
\text { Limit per } 1000\end{array}$ \\
\hline Unspecified Symptoms & $780-799$ & 35 & 2.9 & 2.0 & 4.3 \\
\hline Injury & $800-999$ & 109 & 9.6 & 7.8 & 11.8 \\
\hline -Fractures - Skull, Neck, Trunk & $800-809$ & 5 & 0.4 & 0.2 & 1.1 \\
\hline -Fractures - Upper Limb & $810-819$ & 7 & 0.6 & 0.3 & 1.3 \\
\hline -Fractures - Lower Limb & $820-829$ & 17 & 1.4 & 0.8 & 2.3 \\
\hline -Dislocations & $830-839$ & 17 & 1.5 & 0.9 & 2.5 \\
\hline -Back Sprains \& Strains & $846-847$ & 10 & 1.0 & 0.5 & 2.1 \\
\hline -Other Sprains \& Strains & $840-845,848$ & 26 & 2.6 & 1.7 & 4.1 \\
\hline -Open Wounds & $870-887,890-897$ & 5 & 0.3 & 0.1 & 0.8 \\
\hline -Burns & $940-949$ & 1 & 0.1 & 0.0 & 0.4 \\
\hline -Adverse Reactions to Nonmedical Substances & $980-989$ & 0 & 0 & 0 & 0 \\
\hline -Adverse Reactions to External Causes & $990-995$ & 1 & 0.1 & 0.0 & 0.4 \\
\hline Health Status/Health Service Contact & V01-V82 & 0 & 0 & 0 & 0 \\
\hline Total & & 738 & 65.7 & 60.5 & 71.3 \\
\hline
\end{tabular}

*Standardized to age distribution of 2000 U.S. population.

**Only women aged 18-45 were included in the calculation of the rates for these diagnostic categories. 
Lawrence Livermore National Laboratory 2006

OSHA Data

Appendix L. Number of Workers with at Least One OSHA Event by Gender, Age, and Job Category*

\begin{tabular}{|c|c|c|c|c|c|c|c|c|c|c|c|}
\hline \multirow{3}{*}{ Job Category } & \multicolumn{5}{|c|}{ Women } & \multicolumn{5}{|c|}{ Men } & \multirow[b]{3}{*}{ TOTAL } \\
\hline & \multicolumn{4}{|c|}{ Age Group } & \multirow[b]{2}{*}{ TOTAL } & \multicolumn{4}{|c|}{ Age Group } & \multirow[b]{2}{*}{ TOTAL } & \\
\hline & $16-29$ & 30 - 39 & $40-49$ & $50+$ & & $16-29$ & 30 - 39 & $40-49$ & $50+$ & & \\
\hline Professional & 1 & 2 & 1 & 4 & 8 & 0 & 1 & 5 & 4 & 10 & 18 \\
\hline Administrative Support & 0 & 6 & 14 & 18 & 38 & 0 & 3 & 6 & 15 & 24 & 62 \\
\hline Technical Support & 5 & 2 & 1 & 3 & 11 & 1 & 2 & 10 & 16 & 29 & 40 \\
\hline Service & 1 & 0 & 1 & 3 & 5 & 1 & 3 & 0 & 3 & 7 & 12 \\
\hline Security and Fire & 0 & 0 & 0 & 0 & 0 & 5 & 4 & 9 & 4 & 22 & 22 \\
\hline Crafts & 0 & 0 & 1 & 0 & 1 & 0 & 2 & 5 & 6 & 13 & 14 \\
\hline TOTAL & 7 & 10 & 18 & 28 & 63 & 7 & 15 & 35 & 48 & 105 & 168 \\
\hline
\end{tabular}

*Only those job categories and gender/age combinations with at least one OSHA event appear in this table.

Appendix M. Total Number of Workdays Lost or with Restricted Activity from OSHA Events by Gender and Age

\begin{tabular}{|c|c|c|c|c|c|c|c|c|c|}
\hline \multirow{2}{*}{$\begin{array}{c}\text { Age } \\
\text { Group }\end{array}$} & \multicolumn{3}{|c|}{ Women } & \multicolumn{3}{|c|}{ Men } & \multicolumn{3}{|c|}{ TOTAL } \\
\hline & $\begin{array}{c}\text { Number } \\
\text { of } \\
\text { Events }\end{array}$ & $\begin{array}{c}\text { Days } \\
\text { Restricted }\end{array}$ & $\begin{array}{l}\text { Days } \\
\text { Lost }\end{array}$ & $\begin{array}{c}\text { Number } \\
\text { of } \\
\text { Events }\end{array}$ & $\begin{array}{c}\text { Days } \\
\text { Restricted }\end{array}$ & $\begin{array}{c}\text { Days } \\
\text { Lost }\end{array}$ & $\begin{array}{c}\text { Number } \\
\text { of } \\
\text { Events }\end{array}$ & $\begin{array}{c}\text { Days } \\
\text { Restricted }\end{array}$ & $\begin{array}{l}\text { Days } \\
\text { Lost }\end{array}$ \\
\hline $16-29$ & 7 & 230 & 2 & 7 & 406 & 6 & 14 & 636 & 8 \\
\hline $30-39$ & 10 & 162 & 32 & 15 & 356 & 459 & 25 & 518 & 491 \\
\hline $40-49$ & 18 & 112 & 44 & 36 & 913 & 181 & 54 & 1025 & 225 \\
\hline $50+$ & 29 & 208 & 87 & 50 & 1398 & 577 & 79 & 1606 & 664 \\
\hline TOTAL & 64 & 712 & 165 & 108 & 3073 & 1223 & 172 & 3785 & 1388 \\
\hline
\end{tabular}

Appendix N. Total Number of Workdays Lost or with Restricted Activity from OSHA Events by Gender and Job Category*

\begin{tabular}{|l|r|r|r|r|r|r|r|r|r|}
\hline \multirow{2}{*}{ Job Category } & \multicolumn{4}{|c|}{ Women } & \multicolumn{3}{|c|}{ Men } & \multicolumn{3}{c|}{ Total } \\
\cline { 2 - 10 } & $\begin{array}{c}\text { Number } \\
\text { of } \\
\text { Events }\end{array}$ & $\begin{array}{c}\text { Days } \\
\text { Restricted }\end{array}$ & $\begin{array}{c}\text { Days } \\
\text { Lost }\end{array}$ & $\begin{array}{c}\text { Number } \\
\text { of } \\
\text { Events }\end{array}$ & $\begin{array}{c}\text { Days } \\
\text { Restricted }\end{array}$ & $\begin{array}{c}\text { Dumber } \\
\text { Days } \\
\text { Lost } \\
\text { Events }\end{array}$ & $\begin{array}{c}\text { Days } \\
\text { Restricted }\end{array}$ & $\begin{array}{c}\text { Days } \\
\text { Lost }\end{array}$ \\
\hline Professional & 8 & 0 & 0 & 10 & 37 & 5 & 18 & 37 & 5 \\
\hline Administrative Support & 39 & 238 & 132 & 25 & 487 & 237 & 64 & 725 & 369 \\
\hline Technical Support & 11 & 392 & 20 & 30 & 605 & 79 & 41 & 997 & 99 \\
\hline Service & 5 & 8 & 13 & 7 & 560 & 239 & 12 & 568 & 252 \\
\hline Security and Fire & 0 & 0 & 0 & 22 & 937 & 423 & 22 & 937 & 423 \\
\hline Crafts & 1 & 74 & 0 & 14 & 447 & 240 & 15 & 521 & 240 \\
\hline Total & 64 & 712 & 165 & 108 & 3073 & 1223 & 172 & 3785 & 1388 \\
\hline
\end{tabular}

*Only those job categories with at least one OSHA event appear in this table. 
Lawrence Livermore National Laboratory 2006

OSHA Data

Appendix O. Number of Diagnoses in Each Diagnostic Category by Gender and Age*

\begin{tabular}{|c|c|c|c|c|c|c|}
\hline & & \multicolumn{5}{|c|}{ Women } \\
\hline & & \multicolumn{4}{|c|}{ Age Group } & \multirow[b]{2}{*}{ TOTAL } \\
\hline & & $16-29$ & 30 - 39 & $40-49$ & $50+$ & \\
\hline Diagnostic Category & $\begin{array}{l}\text { ICD-9-CM } \\
\text { code }\end{array}$ & & & & & \\
\hline INFECTIOUS \& PARASITIC DISEASES (DIS) & 001-139 & 0 & 0 & 0 & 1 & 1 \\
\hline -Mycoses & 110-118 & 0 & 0 & 0 & 1 & 1 \\
\hline NERVOUS SYSTEM (NS) \& SENSE ORGANS & 320-389 & 1 & 1 & 0 & 2 & 4 \\
\hline -Disorders of Peripheral NS & $350-359$ & 1 & 1 & 0 & 2 & 4 \\
\hline -Disorders of Eye & $360-379$ & 0 & 0 & 0 & 0 & 0 \\
\hline -Dis of Ear \& Mastoid & $380-389$ & 0 & 0 & 0 & 0 & 0 \\
\hline RESPIRATORY SYSTEM & $460-519$ & 0 & 0 & 0 & 0 & 0 \\
\hline -Other Respiratory Dis & $510-519$ & 0 & 0 & 0 & 0 & 0 \\
\hline SKIN \& SUBCUTANEOUS TISSUE & $680-709$ & 0 & 0 & 0 & 1 & 1 \\
\hline -Other Inflammatory Conditions & 690-698 & 0 & 0 & 0 & 1 & 1 \\
\hline -Other & 700-709 & 0 & 0 & 0 & 0 & 0 \\
\hline MUSCULOSKELETAL \& CONNECTIVE TISSUE & $710-739$ & 18 & 5 & 20 & 19 & 62 \\
\hline -Arthropathies & 710-719 & 15 & 1 & 12 & 11 & 39 \\
\hline -Dorsopathies & $720-724$ & 1 & 0 & 3 & 4 & 8 \\
\hline -Rheumatism, Excluding Back & 725-729 & 2 & 4 & 5 & 4 & 15 \\
\hline SYMPTOMS, SIGNS, \& ILL-DEFINED CONDITIONS & 780-799 & 0 & 1 & 4 & 7 & 12 \\
\hline -Symptoms & 780-789 & 0 & 1 & 4 & 7 & 12 \\
\hline INJURY \& POISONING & $800-999$ & 5 & 8 & 10 & 21 & 44 \\
\hline -Fracture - Neck, Trunk & 805-809 & 0 & 0 & 0 & 0 & 0 \\
\hline -Fracture - Upper Limb & 810-819 & 0 & 0 & 1 & 0 & 1 \\
\hline -Fracture - Lower Limb & $820-829$ & 0 & 0 & 0 & 1 & 1 \\
\hline -Sprains \& Strains - Back & 846-847 & 0 & 1 & 3 & 3 & 7 \\
\hline -Sprains \& Strains - Other & $840-845,848$ & 1 & 0 & 2 & 3 & 6 \\
\hline -Intracranial Injury & $850-854$ & 0 & 0 & 1 & 0 & 1 \\
\hline -Open Wound - Head, Neck, Trunk & $870-879$ & 0 & 0 & 0 & 1 & 1 \\
\hline -Open Wound - Upper Limb & $880-887$ & 0 & 0 & 0 & 0 & 0 \\
\hline -Open Wound - Lower Limb & 890-897 & 0 & 0 & 0 & 0 & 0 \\
\hline -Superficial Injury & 910-919 & 0 & 3 & 0 & 4 & 7 \\
\hline -Contusion & $920-924$ & 0 & 0 & 1 & 3 & 4 \\
\hline -Foreign Body Entering Orifice & 930-939 & 0 & 1 & 0 & 0 & 1 \\
\hline -Burns & 940-949 & 0 & 0 & 0 & 0 & 0 \\
\hline -Injury to Nerves \& Spinal Cord & 950-957 & 0 & 0 & 0 & 0 & 0 \\
\hline -Complications \& Unspecified Injuries & 958-959 & 4 & 3 & 2 & 6 & 15 \\
\hline -Toxic Effects - Non-medicinal & 980-989 & 0 & 0 & 0 & 0 & 0 \\
\hline
\end{tabular}

*Only those diagnostic categories and gender/age combinations with at least one OSHA event appear in this table. 
Lawrence Livermore National Laboratory 2006

OSHA Data

Appendix O. Number of Diagnoses in Each Diagnostic Category by Gender and Age*

\begin{tabular}{|c|c|c|c|c|c|c|c|}
\hline & & \multicolumn{5}{|c|}{ Men } & \multirow[b]{3}{*}{ TOTAL } \\
\hline & & \multicolumn{4}{|c|}{ Age Group } & \multirow[b]{2}{*}{ TOTAL } & \\
\hline & & $16-29$ & 30 - 39 & $40-49$ & $50+$ & & \\
\hline Diagnostic Category & $\begin{array}{l}\text { ICD-9-CM } \\
\text { code }\end{array}$ & & & & & & \\
\hline INFECTIOUS \& PARASITIC DISEASES (DIS) & 001-139 & 0 & 2 & 0 & 0 & 2 & 3 \\
\hline -Mycoses & 110-118 & 0 & 2 & 0 & 0 & 2 & 3 \\
\hline NERVOUS SYSTEM (NS) \& SENSE ORGANS & 320-389 & 1 & 1 & 0 & 1 & 3 & 7 \\
\hline -Disorders of Peripheral NS & $350-359$ & 0 & 1 & 0 & 0 & 1 & 5 \\
\hline -Disorders of Eye & $360-379$ & 1 & 0 & 0 & 0 & 1 & 1 \\
\hline -Dis of Ear \& Mastoid & $380-389$ & 0 & 0 & 0 & 1 & 1 & 1 \\
\hline RESPIRATORY SYSTEM & $460-519$ & 0 & 0 & 1 & 1 & 2 & 2 \\
\hline -Other Respiratory Dis & $510-519$ & 0 & 0 & 1 & 1 & 2 & 2 \\
\hline SKIN \& SUBCUTANEOUS TISSUE & 680-709 & 1 & 1 & 0 & 1 & 3 & 4 \\
\hline -Other Inflammatory Conditions & $690-698$ & 1 & 1 & 0 & 0 & 2 & 3 \\
\hline -Other & 700-709 & 0 & 0 & 0 & 1 & 1 & 1 \\
\hline MUSCULOSKELETAL \& CONNECTIVE TISSUE & $710-739$ & 2 & 5 & 20 & 29 & 56 & 118 \\
\hline -Arthropathies & $710-719$ & 0 & 1 & 8 & 17 & 26 & 65 \\
\hline -Dorsopathies & $720-724$ & 2 & 2 & 8 & 5 & 17 & 25 \\
\hline -Rheumatism, Excluding Back & 725-729 & 0 & 2 & 4 & 7 & 13 & 28 \\
\hline SYMPTOMS, SIGNS, \& ILL-DEFINED CONDITIONS & 780-799 & 0 & 4 & 3 & 6 & 13 & 25 \\
\hline -Symptoms & $780-789$ & 0 & 4 & 3 & 6 & 13 & 25 \\
\hline INJURY \& POISONING & $800-999$ & 6 & 12 & 37 & 49 & 104 & 148 \\
\hline -Fracture - Neck, Trunk & $805-809$ & 0 & 0 & 0 & 1 & 1 & 1 \\
\hline -Fracture - Upper Limb & 810-819 & 0 & 3 & 3 & 3 & 9 & 10 \\
\hline -Fracture - Lower Limb & $820-829$ & 0 & 0 & 0 & 1 & 1 & 2 \\
\hline -Sprains \& Strains - Back & 846-847 & 3 & 2 & 6 & 3 & 14 & 21 \\
\hline -Sprains \& Strains - Other & $840-845,848$ & 0 & 2 & 13 & 18 & 33 & 39 \\
\hline -Intracranial Injury & $850-854$ & 0 & 1 & 1 & 0 & 2 & 3 \\
\hline -Open Wound - Head, Neck, Trunk & 870-879 & 0 & 1 & 1 & 0 & 2 & 3 \\
\hline -Open Wound - Upper Limb & $880-887$ & 0 & 1 & 6 & 4 & 11 & 11 \\
\hline -Open Wound - Lower Limb & 890-897 & 0 & 0 & 1 & 0 & 1 & 1 \\
\hline -Superficial Injury & 910-919 & 0 & 0 & 0 & 2 & 2 & 9 \\
\hline -Contusion & $920-924$ & 1 & 0 & 4 & 4 & 9 & 13 \\
\hline -Foreign Body Entering Orifice & 930-939 & 0 & 0 & 0 & 2 & 2 & 3 \\
\hline -Burns & 940-949 & 0 & 0 & 0 & 2 & 2 & 2 \\
\hline -Injury to Nerves \& Spinal Cord & 950-957 & 1 & 0 & 0 & 0 & 1 & 1 \\
\hline -Complications \& Unspecified Injuries & 958-959 & 1 & 1 & 2 & 9 & 13 & 28 \\
\hline -Toxic Effects - Non-medicinal & 980-989 & 0 & 1 & 0 & 0 & 1 & 1 \\
\hline
\end{tabular}

*Only those diagnostic categories and gender/age combinations with at least one OSHA event appear in this table. 
Lawrence Livermore National Laboratory 2006

OSHA Data

Appendix O. Number of Diagnoses in Each Diagnostic Category by Gender and Age*

\begin{tabular}{|c|c|c|c|c|c|c|c|c|c|c|c|}
\hline & \multicolumn{5}{|c|}{ Women } & \multicolumn{5}{|c|}{ Men } & \multirow[b]{3}{*}{ TOTAL } \\
\hline & \multicolumn{4}{|c|}{ Age Group } & \multirow[b]{2}{*}{ TOTAL } & \multicolumn{4}{|c|}{ Age Group } & \multirow[b]{2}{*}{ TOTAL } & \\
\hline & $16-29$ & \begin{tabular}{|l|l|}
30 & -39 \\
\end{tabular} & $40-49$ & $50+$ & & $16-29$ & $30-39$ & $40-49$ & $50+$ & & \\
\hline $\begin{array}{l}\text { Diagnostic } \\
\text { Category }\end{array}$ & & & & & & & & & & & \\
\hline Total & 24 & 15 & 34 & 51 & 124 & 10 & 25 & 61 & 87 & 183 & 307 \\
\hline
\end{tabular}

*Only those diagnostic categories and gender/age combinations with at least one OSHA event appear in this table. 


\section{Lawrence Livermore National Laboratory 2006}

OSHA Data

Appendix P. Number of Workdays Lost or with Restricted Activity in Each Diagnostic Category by Gender and Age*

\begin{tabular}{|c|c|c|c|c|c|c|c|c|c|}
\hline & & \multicolumn{8}{|c|}{ Women } \\
\hline & & \multicolumn{8}{|c|}{ Age Group } \\
\hline & & \multicolumn{2}{|c|}{$16-29$} & \multicolumn{2}{|c|}{$30-39$} & \multicolumn{2}{|c|}{$40-49$} & \multicolumn{2}{|l|}{$50+$} \\
\hline & & $\begin{array}{c}\text { Days } \\
\text { Restricted }\end{array}$ & $\begin{array}{l}\text { Days } \\
\text { Lost }\end{array}$ & $\begin{array}{c}\text { Days } \\
\text { Restricted }\end{array}$ & $\begin{array}{l}\text { Days } \\
\text { Lost }\end{array}$ & $\begin{array}{c}\text { Days } \\
\text { Restricted }\end{array}$ & $\begin{array}{l}\text { Days } \\
\text { Lost }\end{array}$ & \begin{tabular}{c|} 
Days \\
Restricted
\end{tabular} & $\begin{array}{l}\text { Days } \\
\text { Lost }\end{array}$ \\
\hline Diagnostic Category & $\begin{array}{l}\text { ICD-9-CM } \\
\text { Codes }\end{array}$ & & & & & & & & \\
\hline -Mycoses & $110-118$ & 0 & 0 & 0 & 0 & 0 & 0 & 0 & 13 \\
\hline -Disorders of Peripheral NS & $350-359$ & 0 & 0 & 0 & 0 & 0 & 0 & 0 & 17 \\
\hline -Other Inflammatory Conditions & $690-698$ & 0 & 0 & 0 & 0 & 0 & 0 & 0 & 0 \\
\hline -Arthropathies & $710-719$ & 230 & 2 & 0 & 0 & 74 & 0 & 0 & 12 \\
\hline -Dorsopathies & $720-724$ & 153 & 0 & 0 & 0 & 82 & 0 & 0 & 13 \\
\hline -Rheumatism, Excluding Back & $725-729$ & 0 & 0 & 0 & 13 & 0 & 42 & 0 & 17 \\
\hline -Symptoms & 780-789 & 0 & 0 & 0 & 0 & 8 & 2 & 173 & 7 \\
\hline -Fracture - Upper Limb & 810-819 & 0 & 0 & 0 & 0 & 0 & 0 & 0 & 0 \\
\hline -Fracture - Lower Limb & 820-829 & 0 & 0 & 0 & 0 & 0 & 0 & 0 & 4 \\
\hline -Sprains \& Strains - Back & 846-847 & 0 & 0 & 0 & 1 & 82 & 0 & 0 & 1 \\
\hline -Sprains \& Strains - Other & $840-845,848$ & 0 & 0 & 0 & 0 & 30 & 0 & 0 & 0 \\
\hline -Intracranial Injury & $850-854$ & 0 & 0 & 0 & 0 & 0 & 2 & 0 & 0 \\
\hline -Open Wound - Head, Neck, Trunk & 870-879 & 0 & 0 & 0 & 0 & 0 & 0 & 0 & 0 \\
\hline -Superficial Injury & 910-919 & 0 & 0 & 0 & 0 & 0 & 0 & 0 & 12 \\
\hline -Contusion & $920-924$ & 0 & 0 & 0 & 0 & 0 & 0 & 208 & 13 \\
\hline -Foreign Body Entering Orifice & 930-939 & 0 & 0 & 0 & 0 & 0 & 0 & 0 & 0 \\
\hline -Complications \& Unspecified Injuries & 958-959 & 199 & 0 & 162 & 18 & 0 & 0 & 0 & 27 \\
\hline
\end{tabular}

\begin{tabular}{|c|c|c|c|c|c|c|c|c|c|}
\hline & & \multicolumn{8}{|c|}{ Men } \\
\hline & & \multicolumn{8}{|c|}{ Age Group } \\
\hline & & \multicolumn{2}{|c|}{$16-29$} & \multicolumn{2}{|c|}{$30-39$} & \multicolumn{2}{|c|}{$40-49$} & \multicolumn{2}{|l|}{$50+$} \\
\hline & & $\begin{array}{c}\text { Days } \\
\text { Restricted }\end{array}$ & \begin{tabular}{|l|} 
Days \\
Lost
\end{tabular} & \begin{tabular}{|c|} 
Days \\
Restricted
\end{tabular} & \begin{tabular}{|l|} 
Days \\
Lost
\end{tabular} & \begin{tabular}{|c|} 
Days \\
Restricted
\end{tabular} & \begin{tabular}{|l|} 
Days \\
Lost
\end{tabular} & \begin{tabular}{|c|} 
Days \\
Restricted
\end{tabular} & \begin{tabular}{|l} 
Days \\
Lost
\end{tabular} \\
\hline Diagnostic Category & \begin{tabular}{|l|} 
ICD-9-CM \\
Codes
\end{tabular} & & & & & & & & \\
\hline -Mycoses & $110-118$ & 0 & 0 & 27 & 129 & 0 & 0 & 0 & 0 \\
\hline -Disorders of Peripheral NS & $350-359$ & 0 & 0 & 8 & 0 & 0 & 0 & 0 & 0 \\
\hline -Disorders of Eye & $360-379$ & 2 & 2 & 0 & 0 & 0 & 0 & 0 & 0 \\
\hline -Dis of Ear \& Mastoid & 380-389 & 0 & 0 & 0 & 0 & 0 & 0 & 0 & 0 \\
\hline -Other Respiratory Dis & $510-519$ & 0 & 0 & 0 & 0 & 0 & 7 & 78 & 102 \\
\hline -Other Inflammatory Conditions & $690-698$ & 70 & 0 & 0 & 0 & 0 & 0 & 0 & 0 \\
\hline -Other & 700-709 & 0 & 0 & 0 & 0 & 0 & 0 & 0 & 0 \\
\hline -Arthropathies & $710-719$ & 0 & 0 & 0 & 0 & 363 & 44 & 546 & 345 \\
\hline -Dorsopathies & $720-724$ & 258 & 4 & 138 & 50 & 245 & 2 & 384 & 0 \\
\hline -Rheumatism, Excluding Back & $725-729$ & 0 & 0 & 49 & 87 & 102 & 3 & 129 & 0 \\
\hline
\end{tabular}

(Continued)

*OSHA events with >1 ICD-9-CM code in the same diagnostic category were counted only once. Only those diagnostic categories and gender/age combinations with at least one occurrence appear in this table. 


\section{Lawrence Livermore National Laboratory 2006}

OSHA Data

Appendix P. Number of Workdays Lost or with Restricted Activity in Each Diagnostic Category by Gender and Age*

\begin{tabular}{|c|c|c|c|c|c|c|c|c|c|}
\hline & & \multicolumn{8}{|c|}{ Men } \\
\hline & & \multicolumn{8}{|c|}{ Age Group } \\
\hline & & \multicolumn{2}{|c|}{$16-29$} & \multicolumn{2}{|c|}{$30-39$} & \multicolumn{2}{|c|}{$40-49$} & \multicolumn{2}{|l|}{$50+$} \\
\hline & & $\begin{array}{c}\text { Days } \\
\text { Restricted }\end{array}$ & $\begin{array}{l}\text { Days } \\
\text { Lost }\end{array}$ & \begin{tabular}{|c|} 
Days \\
Restricted
\end{tabular} & $\begin{array}{l}\text { Days } \\
\text { Lost }\end{array}$ & \begin{tabular}{|c|} 
Days \\
Restricted
\end{tabular} & \begin{tabular}{|l|} 
Days \\
Lost
\end{tabular} & $\begin{array}{c}\text { Days } \\
\text { Restricted }\end{array}$ & \begin{tabular}{|l} 
Days \\
Lost
\end{tabular} \\
\hline Diagnostic Category & $\begin{array}{l}\text { ICD-9-CM } \\
\text { Codes }\end{array}$ & & & & & & & & \\
\hline -Symptoms & $780-789$ & 0 & 0 & 130 & 179 & 96 & 2 & 40 & 31 \\
\hline -Fracture - Neck, Trunk & $805-809$ & 0 & 0 & 0 & 0 & 0 & 0 & 0 & 3 \\
\hline -Fracture - Upper Limb & $810-819$ & 0 & 0 & 142 & 0 & 163 & 25 & 0 & 3 \\
\hline -Fracture - Lower Limb & $820-829$ & 0 & 0 & 0 & 0 & 0 & 0 & 0 & 0 \\
\hline -Sprains \& Strains - Back & 846-847 & 265 & 4 & 8 & 180 & 99 & 2 & 204 & 0 \\
\hline -Sprains \& Strains - Other & $840-845,848$ & 0 & 0 & 49 & 87 & 526 & 143 & 867 & 349 \\
\hline -Intracranial Injury & $850-854$ & 0 & 0 & 130 & 50 & 0 & 2 & 0 & 0 \\
\hline -Open Wound - Head, Neck, Trunk & 870-879 & 0 & 0 & 130 & 50 & 0 & 2 & 0 & 0 \\
\hline -Open Wound - Upper Limb & $880-887$ & 0 & 0 & 35 & 0 & 0 & 2 & 0 & 0 \\
\hline -Open Wound - Lower Limb & 890-897 & 0 & 0 & 0 & 0 & 0 & 1 & 0 & 0 \\
\hline -Superficial Injury & 910-919 & 0 & 0 & 0 & 0 & 0 & 0 & 8 & 4 \\
\hline -Contusion & $920-924$ & 12 & 0 & 0 & 0 & 29 & 3 & 141 & 39 \\
\hline -Foreign Body Entering Orifice & $930-939$ & 0 & 0 & 0 & 0 & 0 & 0 & 78 & 102 \\
\hline -Burns & 940-949 & 0 & 0 & 0 & 0 & 0 & 0 & 0 & 0 \\
\hline -Injury to Nerves \& Spinal Cord & $950-957$ & 12 & 0 & 0 & 0 & 0 & 0 & 0 & 0 \\
\hline -Complications \& Unspecified Injuries & 958-959 & 57 & 0 & 0 & 0 & 67 & 22 & 0 & 3 \\
\hline -Toxic Effects - Non-medicinal & 980-989 & 0 & 0 & 0 & 13 & 0 & 0 & 0 & 0 \\
\hline
\end{tabular}

*OSHA events with >1 ICD-9-CM code in the same diagnostic category were counted only once. Only those diagnostic categories and gender/age combinations with at least one occurrence appear in this table. 


\section{Lawrence Livermore National Laboratory 2006}

OSHA Data

Appendix Q. Number of Occurrences in Each Accident Category by Gender and Age*

\begin{tabular}{|c|c|c|c|c|c|c|c|c|c|c|c|c|}
\hline & & \multicolumn{5}{|c|}{ Women } & \multicolumn{5}{|c|}{ Men } & \multirow[b]{3}{*}{ TOTAL } \\
\hline & & \multicolumn{4}{|c|}{ Age Group } & \multirow[b]{2}{*}{ TOTAL } & \multicolumn{4}{|c|}{ Age Group } & \multirow[b]{2}{*}{ TOTAL } & \\
\hline & & $16-29$ & 30 - 39 & $40-49$ & $\begin{array}{c}50 \\
+\end{array}$ & & $16-29$ & $30-39$ & $40-49$ & $\begin{array}{l}50 \\
+\end{array}$ & & \\
\hline Type of Accident & E Codes & & & & & & & & & & & \\
\hline Motor Vehicle Traffic & E810-E819 & 0 & 1 & 0 & 1 & 2 & 0 & 0 & 1 & 0 & 1 & 3 \\
\hline Other Road Vehicle & E826-E829 & 0 & 1 & 0 & 0 & 1 & 0 & 1 & 2 & 1 & 4 & 5 \\
\hline Falls & E880-E888 & 0 & 1 & 5 & 5 & 11 & 0 & 3 & 3 & 5 & 11 & 22 \\
\hline Natural/Environmental Factors & E900-E909 & 0 & 0 & 0 & 0 & 0 & 0 & 1 & 0 & 2 & 3 & 3 \\
\hline $\begin{array}{l}\text { Submersion/Suffocation/Foreign } \\
\text { Bodies }\end{array}$ & E910-E915 & 0 & 1 & 0 & 0 & 1 & 0 & 0 & 0 & 2 & 2 & 3 \\
\hline Other Accidents & E916-E928 & 7 & 6 & 13 & 21 & 47 & 5 & 6 & 29 & 36 & 76 & 123 \\
\hline $\begin{array}{l}\text { Adverse } \\
\text { Effect - Medicinal/Biological }\end{array}$ & \begin{tabular}{|l|} 
E930-E949 \\
\end{tabular} & 0 & 0 & 0 & 0 & 0 & 0 & 1 & 0 & 0 & 1 & 1 \\
\hline
\end{tabular}

*Only those accident types and gender/age combinations with at least one occurrence appear in this table.

Appendix R. Number of Workdays Lost or with Restricted Activity in Each Accident Category by Gender and Age*

\begin{tabular}{|c|c|c|c|c|c|c|c|c|c|}
\hline & & \multicolumn{8}{|c|}{ Women } \\
\hline & & \multicolumn{8}{|c|}{ Age Group } \\
\hline & & \multicolumn{2}{|c|}{$16-29$} & \multicolumn{2}{|c|}{$30-39$} & \multicolumn{2}{|c|}{$40-49$} & \multicolumn{2}{|l|}{$\mathbf{5 0 +}$} \\
\hline & & \begin{tabular}{|c} 
Days \\
Restricted
\end{tabular} & \begin{tabular}{|l|} 
Days \\
Lost
\end{tabular} & \begin{tabular}{|c|} 
Days \\
Restricted
\end{tabular} & \begin{tabular}{|l|} 
Days \\
Lost
\end{tabular} & \begin{tabular}{c|} 
Days \\
Restricted
\end{tabular} & $\begin{array}{l}\text { Days } \\
\text { Lost }\end{array}$ & \begin{tabular}{|c|} 
Days \\
Restricted
\end{tabular} & \begin{tabular}{|l} 
Days \\
Lost \\
\end{tabular} \\
\hline Type of Accident & E Codes & & & & & & & & \\
\hline Motor Vehicle Traffic & E810-E819 & 0 & 0 & 0 & 0 & 0 & 0 & 173 & 7 \\
\hline Other Road Vehicle & \begin{tabular}{|l|} 
E826-E829 \\
\end{tabular} & 0 & 0 & 0 & 0 & 0 & 0 & 0 & 0 \\
\hline Falls & \begin{tabular}{|l|} 
E880-E888 \\
\end{tabular} & 0 & 0 & 0 & 1 & 30 & 0 & 0 & 4 \\
\hline Submersion/Suffocation/Foreign Bodies & E910-E915 & 0 & 0 & 0 & 0 & 0 & 0 & 0 & 0 \\
\hline Other Accidents & E916-E928 & 230 & 2 & 162 & 31 & 82 & 44 & 35 & 63 \\
\hline
\end{tabular}

\begin{tabular}{|c|c|c|c|c|c|c|c|c|c|}
\hline & & \multicolumn{8}{|c|}{ Men } \\
\hline & & \multicolumn{8}{|c|}{ Age Group } \\
\hline & & \multicolumn{2}{|c|}{$16-29$} & \multicolumn{2}{|c|}{$30-39$} & \multicolumn{2}{|c|}{$40-49$} & \multicolumn{2}{|l|}{$50+$} \\
\hline & & $\begin{array}{c}\text { Days } \\
\text { Restricted }\end{array}$ & \begin{tabular}{|l|} 
Days \\
Lost
\end{tabular} & \begin{tabular}{|c|} 
Days \\
Restricted
\end{tabular} & \begin{tabular}{|l|} 
Days \\
Lost \\
\end{tabular} & \begin{tabular}{|c|} 
Days \\
Restricted
\end{tabular} & \begin{tabular}{|l|} 
Days \\
Lost \\
\end{tabular} & \begin{tabular}{|c|} 
Days \\
Restricted
\end{tabular} & $\begin{array}{l}\text { Days } \\
\text { Lost }\end{array}$ \\
\hline Type of Accident & E Codes & \multirow[b]{2}{*}{0} & \multirow[b]{2}{*}{0} & \multirow[b]{2}{*}{0} & \multirow[b]{2}{*}{0} & \multirow[b]{2}{*}{0} & \multirow[b]{2}{*}{2} & \multirow[b]{2}{*}{0} & \multirow[b]{2}{*}{0} \\
\hline Motor Vehicle Traffic & E810-E819 & & & & & & & & \\
\hline Other Road Vehicle & E826-E829 & 0 & 0 & 37 & 0 & 67 & 22 & 0 & 3 \\
\hline Falls & E880-E888 & 0 & 0 & 49 & 267 & 53 & 1 & 9 & 0 \\
\hline Natural/Environmental Factors & E900-E909 & 0 & 0 & 0 & 13 & 0 & 0 & 8 & 4 \\
\hline Submersion/Suffocation/Foreign Bodies & E910-E915 & 0 & 0 & 0 & 0 & 0 & 0 & 78 & 102 \\
\hline Other Accidents & E916-E928 & 334 & 4 & 235 & 50 & 793 & 149 & 1303 & 468 \\
\hline Adverse Effect - Medicinal/Biological & E930-E949 & 0 & 0 & 0 & 0 & 0 & 0 & 0 & 0 \\
\hline
\end{tabular}

*OSHA events with >1 E code in the same accident type were counted only once. Only those accident types and gender/age combinations with at least one occurrence appear in this table. 


\section{Lawrence Livermore National Laboratory 2006}

OSHA Data

Appendix S. Number of Diagnoses in Each Diagnostic Category by Gender and Job Category*

\begin{tabular}{|c|c|c|c|c|c|c|c|}
\hline & & \multicolumn{6}{|c|}{ Women } \\
\hline & & \multicolumn{5}{|c|}{ Job Category } & \multirow[b]{2}{*}{ TOTAL } \\
\hline & & Professional & $\begin{array}{c}\text { Administrative } \\
\text { Support }\end{array}$ & $\begin{array}{l}\text { Technical } \\
\text { Support }\end{array}$ & Service & Crafts & \\
\hline Diagnostic Category & $\begin{array}{l}\text { ICD-9-CM } \\
\text { Code }\end{array}$ & & & & & & \\
\hline INFECTIOUS \& PARASITIC DISEASES (DIS) & 001-139 & 0 & 0 & 0 & 1 & 0 & 1 \\
\hline -Mycoses & 110-118 & 0 & 0 & 0 & 1 & 0 & 1 \\
\hline NERVOUS SYSTEM (NS) \& SENSE ORGANS & 320-389 & 1 & 3 & 0 & 0 & 0 & 4 \\
\hline -Disorders of Peripheral NS & $350-359$ & 1 & 3 & 0 & 0 & 0 & 4 \\
\hline SKIN \& SUBCUTANEOUS TISSUE & 680-709 & 0 & 0 & 0 & 1 & 0 & 1 \\
\hline -Other Inflammatory Conditions & 690-698 & 0 & 0 & 0 & 1 & 0 & 1 \\
\hline MUSCULOSKELETAL \& CONNECTIVE TISSUE & $710-739$ & 10 & 31 & 16 & 3 & 2 & 62 \\
\hline -Arthropathies & 710-719 & 6 & 18 & 12 & 2 & 1 & 39 \\
\hline -Dorsopathies & 720-724 & 2 & 3 & 1 & 1 & 1 & 8 \\
\hline -Rheumatism, Excluding Back & 725-729 & 2 & 10 & 3 & 0 & 0 & 15 \\
\hline $\begin{array}{l}\text { SYMPTOMS, SIGNS, \& ILL-DEFINED } \\
\text { CONDITIONS }\end{array}$ & 780-799 & 2 & 7 & 2 & 1 & 0 & 12 \\
\hline -Symptoms & 780-789 & 2 & 7 & 2 & 1 & 0 & 12 \\
\hline INJURY \& POISONING & 800-999 & 4 & 27 & 9 & 3 & 1 & 44 \\
\hline -Fracture - Upper Limb & 810-819 & 0 & 1 & 0 & 0 & 0 & 1 \\
\hline -Fracture - Lower Limb & $820-829$ & 0 & 1 & 0 & 0 & 0 & 1 \\
\hline -Sprains \& Strains - Back & 846-847 & 1 & 4 & 0 & 1 & 1 & 7 \\
\hline -Sprains \& Strains - Other & $\begin{array}{l}840-845 \\
848\end{array}$ & 1 & 3 & 1 & 1 & 0 & 6 \\
\hline -Intracranial Injury & $850-854$ & 0 & 1 & 0 & 0 & 0 & 1 \\
\hline -Open Wound - Head, Neck, Trunk & 870-879 & 0 & 1 & 0 & 0 & 0 & 1 \\
\hline -Superficial Injury & 910-919 & 1 & 6 & 0 & 0 & 0 & 7 \\
\hline -Contusion & 920-924 & 0 & 4 & 0 & 0 & 0 & 4 \\
\hline -Foreign Body Entering Orifice & 930-939 & 1 & 0 & 0 & 0 & 0 & 1 \\
\hline -Complications \& Unspecified Injuries & 958-959 & 0 & 6 & 8 & 1 & 0 & 15 \\
\hline
\end{tabular}

\begin{tabular}{|l|r|r|r|r|r|r|}
\hline \multirow{4}{*}{} & \multicolumn{5}{|c|}{ Wob Category } & \\
\cline { 2 - 6 } & \multicolumn{5}{|c|}{ Jomen } \\
\cline { 2 - 6 } & Professional & $\begin{array}{c}\text { Administrative } \\
\text { Support }\end{array}$ & $\begin{array}{l}\text { Technical } \\
\text { Support }\end{array}$ & Service & Crafts & TOTAL \\
\hline $\begin{array}{l}\text { Diagnostic } \\
\text { Category }\end{array}$ & & & & & & \\
\cline { 1 - 7 } Total & 17 & 68 & 27 & 9 & 3 & 124 \\
\hline
\end{tabular}

*Only those diagnostic categories and gender/job category combinations with at least one occurrence appear in this table. 


\section{Lawrence Livermore National Laboratory 2006}

OSHA Data

Appendix S. Number of Diagnoses in Each Diagnostic Category by Gender and Job Category*

\begin{tabular}{|c|c|c|c|c|c|c|c|c|}
\hline & & \multicolumn{7}{|c|}{ Men } \\
\hline & & \multicolumn{6}{|c|}{ Job Category } & \multirow[b]{2}{*}{ TOTAL } \\
\hline & & Professional & $\begin{array}{l}\text { Administrative } \\
\text { Support }\end{array}$ & $\begin{array}{l}\text { Technical } \\
\text { Support }\end{array}$ & Service & $\begin{array}{c}\text { Security } \\
\text { and } \\
\text { Fire }\end{array}$ & Crafts & \\
\hline Diagnostic Category & \begin{tabular}{|l|} 
ICD-9-CM \\
Code
\end{tabular} & & & & & & & \\
\hline $\begin{array}{l}\text { INFECTIOUS \& PARASITIC DISEASES } \\
\text { (DIS) }\end{array}$ & 001-139 & 0 & 0 & 0 & 0 & 1 & 1 & 2 \\
\hline -Mycoses & $110-118$ & 0 & 0 & 0 & 0 & 1 & 1 & 2 \\
\hline $\begin{array}{l}\text { NERVOUS SYSTEM (NS) \& SENSE } \\
\text { ORGANS }\end{array}$ & 320-389 & 0 & 1 & 0 & 0 & 2 & 0 & 3 \\
\hline -Disorders of Peripheral NS & $350-359$ & 0 & 0 & 0 & 0 & 1 & 0 & 1 \\
\hline -Disorders of Eye & $360-379$ & 0 & 0 & 0 & 0 & 1 & 0 & 1 \\
\hline -Dis of Ear \& Mastoid & 380-389 & 0 & 1 & 0 & 0 & 0 & 0 & 1 \\
\hline RESPIRATORY SYSTEM & $460-519$ & 0 & 1 & 0 & 0 & 1 & 0 & 2 \\
\hline -Other Respiratory Dis & $510-519$ & 0 & 1 & 0 & 0 & 1 & 0 & 2 \\
\hline SKIN \& SUBCUTANEOUS TISSUE & $680-709$ & 0 & 1 & 1 & 1 & 0 & 0 & 3 \\
\hline -Other Inflammatory Conditions & $690-698$ & 0 & 1 & 0 & 1 & 0 & 0 & 2 \\
\hline -Other & 700-709 & 0 & 0 & 1 & 0 & 0 & 0 & 1 \\
\hline $\begin{array}{l}\text { MUSCULOSKELETAL \& CONNECTIVE } \\
\text { TISSUE }\end{array}$ & 710-739 & 7 & 8 & 16 & 7 & 11 & 7 & 56 \\
\hline -Arthropathies & $710-719$ & 3 & 5 & 8 & 2 & 4 & 4 & 26 \\
\hline -Dorsopathies & $720-724$ & 2 & 2 & 6 & 2 & 4 & 1 & 17 \\
\hline -Rheumatism, Excluding Back & 725-729 & 2 & 1 & 2 & 3 & 3 & 2 & 13 \\
\hline $\begin{array}{l}\text { SYMPTOMS, SIGNS, \& ILL-DEFINED } \\
\text { CONDITIONS }\end{array}$ & $780-799$ & 2 & 2 & 3 & 2 & 4 & 0 & 13 \\
\hline -Symptoms & 780-789 & 2 & 2 & 3 & 2 & 4 & 0 & 13 \\
\hline INJURY \& POISONING & $800-999$ & 14 & 20 & 29 & 7 & 20 & 14 & 104 \\
\hline -Fracture - Neck, Trunk & $805-809$ & 1 & 0 & 0 & 0 & 0 & 0 & 1 \\
\hline -Fracture - Upper Limb & $810-819$ & 3 & 1 & 2 & 0 & 1 & 2 & 9 \\
\hline -Fracture - Lower Limb & $820-829$ & 1 & 0 & 0 & 0 & 0 & 0 & 1 \\
\hline -Sprains \& Strains - Back & 846-847 & 1 & 1 & 4 & 0 & 7 & 1 & 14 \\
\hline -Sprains \& Strains - Other & $\begin{array}{l}840-845 \\
848\end{array}$ & 0 & 7 & 7 & 4 & 9 & 6 & 33 \\
\hline -Intracranial Injury & $850-854$ & 1 & 0 & 0 & 1 & 0 & 0 & 2 \\
\hline -Open Wound - Head, Neck, Trunk & $870-879$ & 1 & 0 & 0 & 1 & 0 & 0 & 2 \\
\hline -Open Wound - Upper Limb & $880-887$ & 1 & 1 & 6 & 0 & 1 & 2 & 11 \\
\hline -Open Wound - Lower Limb & 890-897 & 0 & 1 & 0 & 0 & 0 & 0 & 1 \\
\hline -Superficial Injury & 910-919 & 0 & 0 & 2 & 0 & 0 & 0 & 2 \\
\hline -Contusion & $920-924$ & 2 & 2 & 3 & 0 & 1 & 1 & 9 \\
\hline -Foreign Body Entering Orifice & 930-939 & 1 & 1 & 0 & 0 & 0 & 0 & 2 \\
\hline -Burns & 940-949 & 0 & 1 & 0 & 0 & 0 & 1 & 2 \\
\hline
\end{tabular}

(Continued)

*Only those diagnostic categories and gender/job category combinations with at least one occurrence appear in this table. 


\section{Lawrence Livermore National Laboratory 2006}

OSHA Data

Appendix S. Number of Diagnoses in Each Diagnostic Category by Gender and Job Category*

\begin{tabular}{|c|c|c|c|c|c|c|c|c|}
\hline & & \multicolumn{7}{|c|}{ Men } \\
\hline & & \multicolumn{6}{|c|}{ Job Category } & \multirow[b]{2}{*}{ TOTAL } \\
\hline & & Professional & $\begin{array}{c}\text { Administrative } \\
\text { Support }\end{array}$ & $\begin{array}{l}\text { Technical } \\
\text { Support }\end{array}$ & Service & $\begin{array}{c}\text { Security } \\
\text { and } \\
\text { Fire }\end{array}$ & Crafts & \\
\hline Diagnostic Category & $\begin{array}{l}\text { ICD-9-CM } \\
\text { Code }\end{array}$ & \multirow[b]{2}{*}{0} & \multirow[b]{2}{*}{0} & \multirow[b]{2}{*}{0} & \multirow[b]{2}{*}{0} & \multirow[b]{2}{*}{1} & \multirow[b]{2}{*}{0} & \multirow[b]{2}{*}{1} \\
\hline -Injury to Nerves \& Spinal Cord & $950-957$ & & & & & & & \\
\hline -Complications \& Unspecified Injuries & 958-959 & 2 & 5 & 5 & 0 & 0 & 1 & 13 \\
\hline -Toxic Effects - Non-medicinal & 980-989 & 0 & 0 & 0 & 1 & 0 & 0 & 1 \\
\hline
\end{tabular}

\begin{tabular}{|c|c|c|c|c|c|c|c|}
\hline & \multicolumn{7}{|c|}{ Men } \\
\hline & \multicolumn{6}{|c|}{ Job Category } & \multirow[b]{2}{*}{ TOTAL } \\
\hline & Professional & $\begin{array}{c}\text { Administrative } \\
\text { Support }\end{array}$ & $\begin{array}{l}\text { Technical } \\
\text { Support }\end{array}$ & Service & $\begin{array}{c}\text { Security } \\
\text { and } \\
\text { Fire }\end{array}$ & Crafts & \\
\hline $\begin{array}{l}\text { Diagnostic } \\
\text { Category }\end{array}$ & \multirow[b]{2}{*}{23} & \multirow[b]{2}{*}{33} & \multirow[b]{2}{*}{49} & \multirow[b]{2}{*}{17} & \multirow[b]{2}{*}{39} & \multirow[b]{2}{*}{22} & \multirow[b]{2}{*}{183} \\
\hline Total & & & & & & & \\
\hline
\end{tabular}

*Only those diagnostic categories and gender/job category combinations with at least one occurrence appear in this table. 


\section{Lawrence Livermore National Laboratory 2006}

OSHA Data

Appendix T. Number of Workdays Lost or with Restricted Activity in Each Diagnostic Category by Gender and Job Category*

\begin{tabular}{|c|c|c|c|c|c|c|c|}
\hline & & \multicolumn{6}{|c|}{ Women } \\
\hline & & \multicolumn{6}{|c|}{ Job Category } \\
\hline & & \multicolumn{2}{|c|}{ Professional } & \multicolumn{2}{|c|}{$\begin{array}{l}\text { Administrative } \\
\text { Support }\end{array}$} & \multicolumn{2}{|c|}{$\begin{array}{l}\text { Technical } \\
\text { Support }\end{array}$} \\
\hline & & \begin{tabular}{|c|} 
Days \\
Restricted
\end{tabular} & $\begin{array}{l}\text { Days } \\
\text { Lost }\end{array}$ & \begin{tabular}{|c|} 
Days \\
Restricted
\end{tabular} & $\begin{array}{l}\text { Days } \\
\text { Lost }\end{array}$ & $\begin{array}{c}\text { Days } \\
\text { Restricted }\end{array}$ & $\begin{array}{l}\text { Days } \\
\text { Lost }\end{array}$ \\
\hline Diagnostic Category & \begin{tabular}{|l|} 
ICD-9-CM \\
Codes
\end{tabular} & & & & & & \\
\hline -Mycoses & 110-118 & 0 & 0 & 0 & 0 & 0 & 0 \\
\hline -Disorders of Peripheral NS & $350-359$ & 0 & 0 & 0 & 17 & 0 & 0 \\
\hline -Other Inflammatory Conditions & $690-698$ & 0 & 0 & 0 & 0 & 0 & $\overline{0}$ \\
\hline -Arthropathies & 710-719 & 0 & 0 & 0 & 12 & 230 & 2 \\
\hline -Dorsopathies & $720-724$ & 0 & 0 & 0 & 13 & 153 & 0 \\
\hline -Rheumatism, Excluding Back & $725-729$ & 0 & 0 & 0 & 72 & 0 & $\overline{0}$ \\
\hline -Symptoms & 780-789 & 0 & 0 & 173 & 9 & 0 & $\overline{0}$ \\
\hline -Fracture - Upper Limb & 810-819 & 0 & 0 & 0 & 0 & 0 & 0 \\
\hline -Fracture - Lower Limb & 820-829 & 0 & 0 & 0 & 4 & 0 & 0 \\
\hline -Sprains \& Strains - Back & 846-847 & 0 & 0 & 0 & 2 & 0 & 0 \\
\hline -Sprains \& Strains - Other & $840-845,848$ & 0 & 0 & 30 & 0 & 0 & 0 \\
\hline -Intracranial Injury & $850-854$ & 0 & 0 & 0 & 2 & 0 & 0 \\
\hline -Open Wound - Head, Neck, Trunk & $870-879$ & 0 & 0 & 0 & 0 & 0 & 0 \\
\hline -Superficial Injury & $910-919$ & 0 & 0 & 0 & 12 & 0 & 0 \\
\hline -Contusion & $920-924$ & 0 & 0 & 208 & 13 & 0 & 0 \\
\hline -Foreign Body Entering Orifice & 930-939 & 0 & 0 & 0 & 0 & 0 & 0 \\
\hline -Complications \& Unspecified Injuries & 958-959 & 0 & 0 & 0 & 27 & 361 & 18 \\
\hline
\end{tabular}

*OSHA events with >1 ICD-9-CM code in the same diagnostic category were counted only once. Only those diagnostic categories and gender/job category combinations with at least one occurrence appear in this table. 


\section{Lawrence Livermore National Laboratory 2006}

OSHA Data

Appendix T. Number of Workdays Lost or with Restricted Activity in Each Diagnostic Category by Gender and Job Category*

\begin{tabular}{|c|c|c|c|c|c|}
\hline & & \multicolumn{4}{|c|}{ Women } \\
\hline & & \multicolumn{4}{|c|}{ Job Category } \\
\hline & & \multicolumn{2}{|c|}{ Service } & \multicolumn{2}{|c|}{ Crafts } \\
\hline & & $\begin{array}{c}\text { Days } \\
\text { Restricted }\end{array}$ & \begin{tabular}{|l|} 
Days \\
Lost
\end{tabular} & \begin{tabular}{c|} 
Days \\
Restricted
\end{tabular} & $\begin{array}{l}\text { Days } \\
\text { Lost }\end{array}$ \\
\hline Diagnostic Category & \begin{tabular}{|l|} 
ICD-9-CM \\
Codes
\end{tabular} & & & & \\
\hline -Mycoses & $110-118$ & 0 & 13 & 0 & 0 \\
\hline -Disorders of Peripheral NS & $350-359$ & 0 & 0 & 0 & 0 \\
\hline -Other Inflammatory Conditions & 690-698 & 0 & 0 & 0 & 0 \\
\hline -Arthropathies & $710-719$ & 0 & 0 & 74 & 0 \\
\hline -Dorsopathies & $720-724$ & 8 & 0 & 74 & 0 \\
\hline -Rheumatism, Excluding Back & $725-729$ & 0 & 0 & 0 & 0 \\
\hline -Symptoms & 780-789 & 8 & 0 & 0 & 0 \\
\hline -Fracture - Upper Limb & 810-819 & 0 & 0 & 0 & 0 \\
\hline -Fracture - Lower Limb & $820-829$ & 0 & 0 & 0 & 0 \\
\hline -Sprains \& Strains - Back & $846-847$ & 8 & 0 & 74 & 0 \\
\hline -Sprains \& Strains - Other & $840-845,848$ & 0 & 0 & 0 & 0 \\
\hline -Intracranial Injury & $850-854$ & 0 & 0 & 0 & 0 \\
\hline -Open Wound - Head, Neck, Trunk & $870-879$ & 0 & 0 & 0 & 0 \\
\hline -Superficial Injury & $910-919$ & 0 & 0 & 0 & 0 \\
\hline -Contusion & $920-924$ & 0 & 0 & 0 & 0 \\
\hline -Foreign Body Entering Orifice & $930-939$ & 0 & 0 & 0 & 0 \\
\hline -Complications \& Unspecified Injuries & 958-959 & 0 & 0 & 0 & 0 \\
\hline
\end{tabular}

*OSHA events with >1 ICD-9-CM code in the same diagnostic category were counted only once. Only those diagnostic categories and gender/job category combinations with at least one occurrence appear in this table. 


\section{Lawrence Livermore National Laboratory 2006}

OSHA Data

Appendix T. Number of Workdays Lost or with Restricted Activity in Each Diagnostic Category by Gender and Job Category*

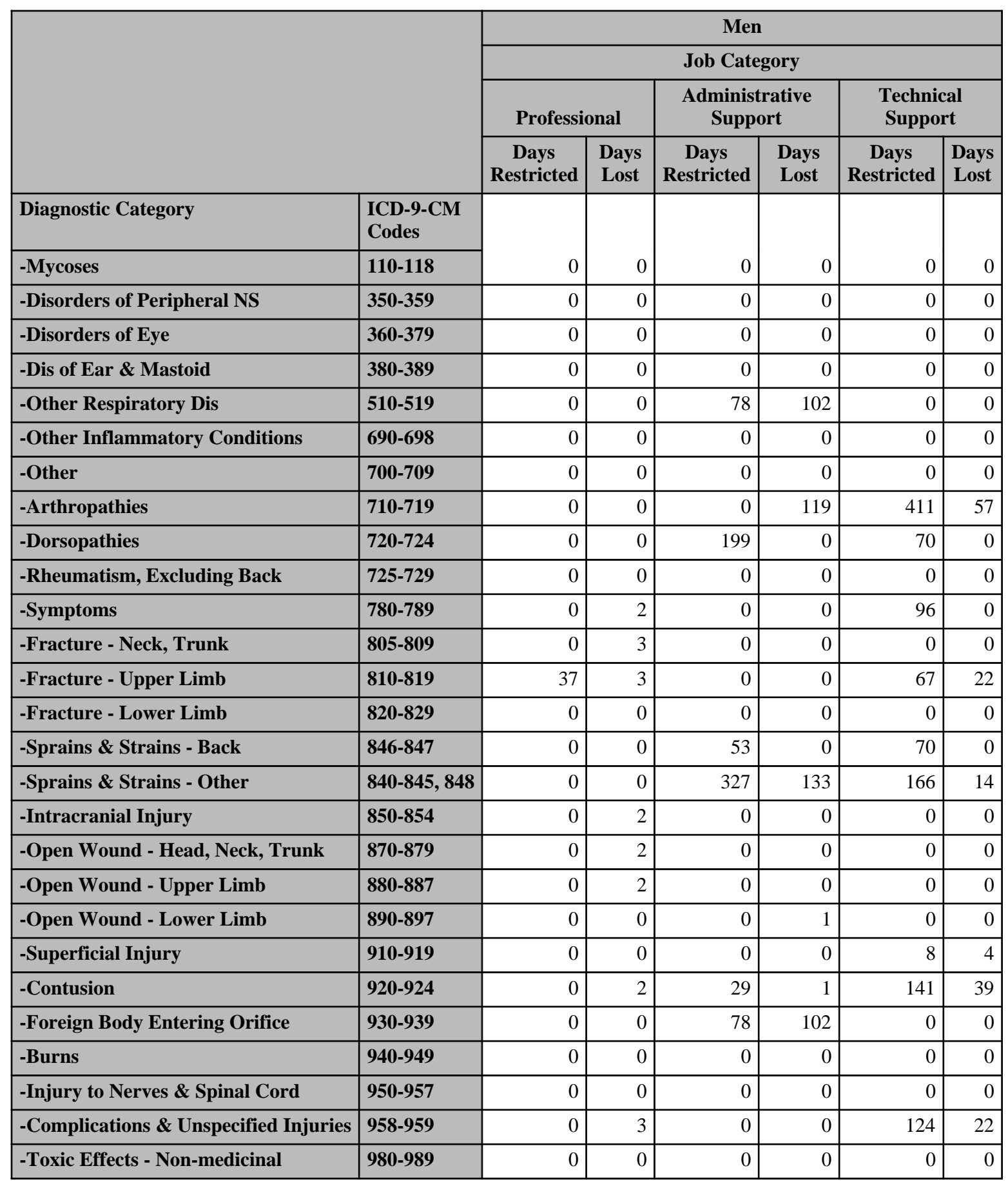

*OSHA events with >1 ICD-9-CM code in the same diagnostic category were counted only once. Only those diagnostic categories and gender/job category combinations with at least one occurrence appear in this table. 


\section{Lawrence Livermore National Laboratory 2006}

OSHA Data

Appendix T. Number of Workdays Lost or with Restricted Activity in Each Diagnostic Category by Gender and Job Category*

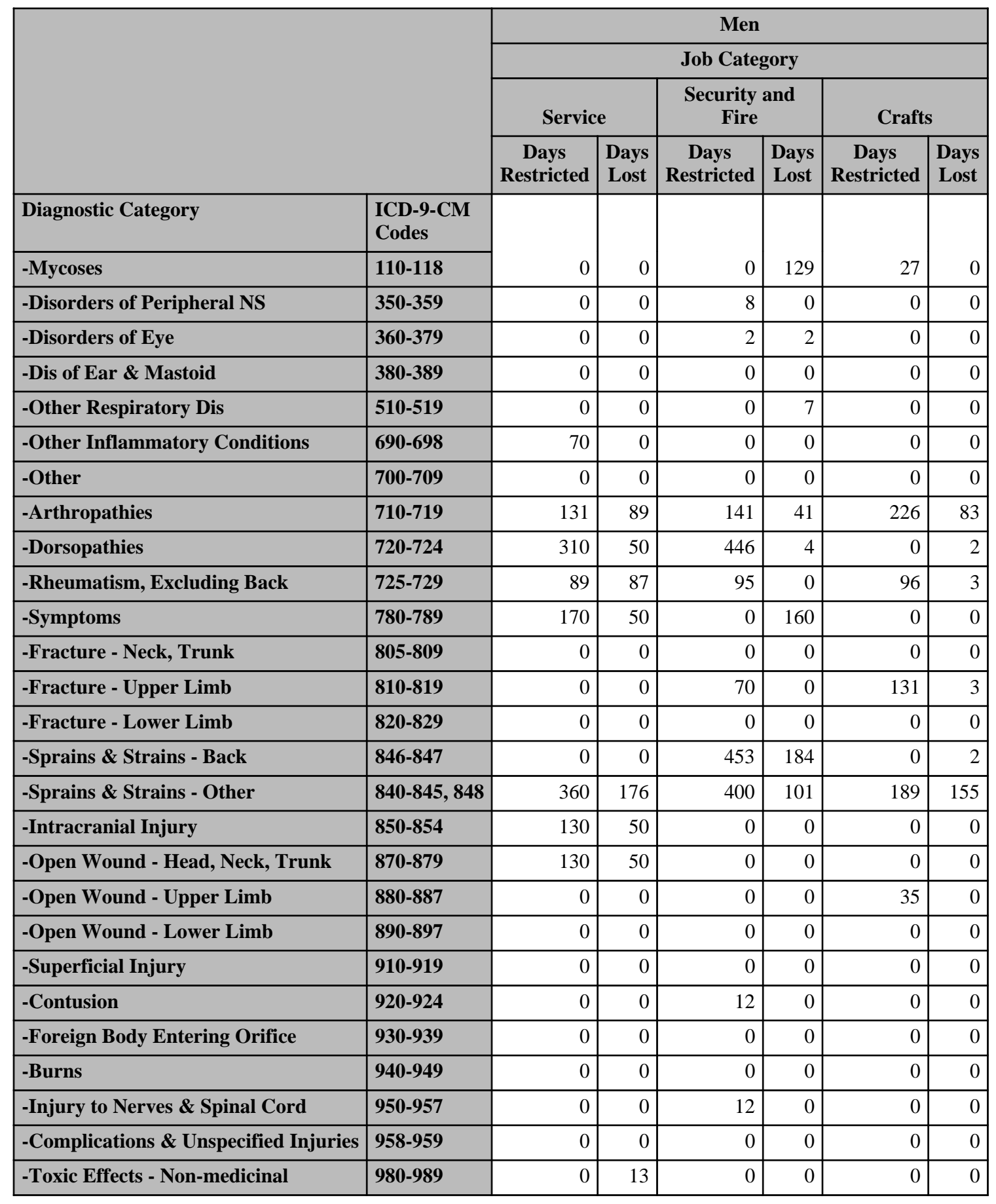

*OSHA events with >1 ICD-9-CM code in the same diagnostic category were counted only once. Only those diagnostic categories and gender/job category combinations with at least one occurrence appear in this table. 


\section{Lawrence Livermore National Laboratory 2006}

OSHA Data

Appendix U. Number of Occurrences in Each Accident Category by Gender and Job Category*

\begin{tabular}{|c|c|c|c|c|c|c|c|}
\hline & & \multicolumn{6}{|c|}{ Women } \\
\hline & & \multicolumn{5}{|c|}{ Job Category } & \multirow[b]{2}{*}{ TOTAL } \\
\hline & & Professional & $\begin{array}{c}\text { Administrative } \\
\text { Support }\end{array}$ & \begin{tabular}{|l|} 
Technical \\
Support
\end{tabular} & Service & Crafts & \\
\hline Type of Accident & E CODES & \multirow[b]{2}{*}{1} & \multirow[b]{2}{*}{1} & \multirow[b]{2}{*}{0} & \multirow[b]{2}{*}{0} & \multirow[b]{2}{*}{0} & \multirow[b]{2}{*}{2} \\
\hline Motor Vehicle Traffic & E810-E819 & & & & & & \\
\hline Other Road Vehicle & E826-E829 & 0 & 1 & 0 & 0 & 0 & 1 \\
\hline Falls & \begin{tabular}{|l|} 
E880-E888 \\
\end{tabular} & 1 & 9 & 1 & 0 & 0 & 11 \\
\hline Submersion/Suffocation/Foreign Bodies & E910-E915 & 1 & 0 & 0 & 0 & 0 & 1 \\
\hline Other Accidents & E916-E928 & 5 & 28 & 10 & 3 & 1 & 47 \\
\hline
\end{tabular}

\begin{tabular}{|c|c|c|c|c|c|c|c|c|}
\hline & & \multicolumn{7}{|c|}{ Men } \\
\hline & & \multicolumn{6}{|c|}{ Job Category } & \multirow[b]{2}{*}{ TOTAL } \\
\hline & & Professional & $\begin{array}{c}\text { Administrative } \\
\text { Support }\end{array}$ & $\begin{array}{l}\text { Technical } \\
\text { Support }\end{array}$ & Service & $\begin{array}{c}\text { Security } \\
\text { and } \\
\text { Fire }\end{array}$ & Crafts & \\
\hline Type of Accident & E CODES & 1 & 0 & 0 & 0 & 0 & 0 & 1 \\
\hline Falls & E880-E888 & 1 & 2 & 3 & 1 & 1 & 3 & 11 \\
\hline Natural/Environmental Factors & E900-E909 & 0 & 0 & 2 & 1 & 0 & 0 & 3 \\
\hline Submersion/Suffocation/Foreign Bodies & E910-E915 & 1 & 1 & 0 & 0 & 0 & 0 & 2 \\
\hline Other Accidents & E916-E928 & 4 & 20 & 21 & 4 & 17 & 10 & 76 \\
\hline
\end{tabular}

*Only those accident types and gender/job category combinations with at least one occurrence appear in this table. 
Lawrence Livermore National Laboratory 2006

OSHA Data

Appendix V. Number of Workdays Lost or with Restricted Activity in Each Accident Category by Gender and Job Category*

\begin{tabular}{|c|c|c|c|c|c|c|c|}
\hline & & \multicolumn{6}{|c|}{ Women } \\
\hline & & \multicolumn{6}{|c|}{ Job Category } \\
\hline & & \multicolumn{2}{|c|}{ Professional } & \multicolumn{2}{|c|}{$\begin{array}{l}\text { Administrative } \\
\text { Support }\end{array}$} & \multicolumn{2}{|c|}{$\begin{array}{l}\text { Technical } \\
\text { Support }\end{array}$} \\
\hline & & $\begin{array}{c}\text { Days } \\
\text { Restricted }\end{array}$ & $\begin{array}{l}\text { Days } \\
\text { Lost }\end{array}$ & $\begin{array}{c}\text { Days } \\
\text { Restricted }\end{array}$ & $\begin{array}{l}\text { Days } \\
\text { Lost }\end{array}$ & $\begin{array}{c}\text { Days } \\
\text { Restricted }\end{array}$ & \begin{tabular}{|l} 
Days \\
Lost
\end{tabular} \\
\hline Type of Accident & E Codes & \multirow[b]{2}{*}{0} & \multirow[b]{2}{*}{0} & \multirow[b]{2}{*}{173} & \multirow[b]{2}{*}{7} & \multirow[b]{2}{*}{0} & \multirow[b]{2}{*}{0} \\
\hline Motor Vehicle Traffic & E810-E819 & & & & & & \\
\hline Other Road Vehicle & E826-E829 & 0 & 0 & 0 & 0 & 0 & 0 \\
\hline Falls & E880-E888 & 0 & 0 & 30 & 5 & 0 & 0 \\
\hline Submersion/Suffocation/Foreign Bodies & E910-E915 & 0 & 0 & 0 & 0 & 0 & 0 \\
\hline Other Accidents & E916-E928 & 0 & 0 & 35 & 120 & 392 & 20 \\
\hline
\end{tabular}

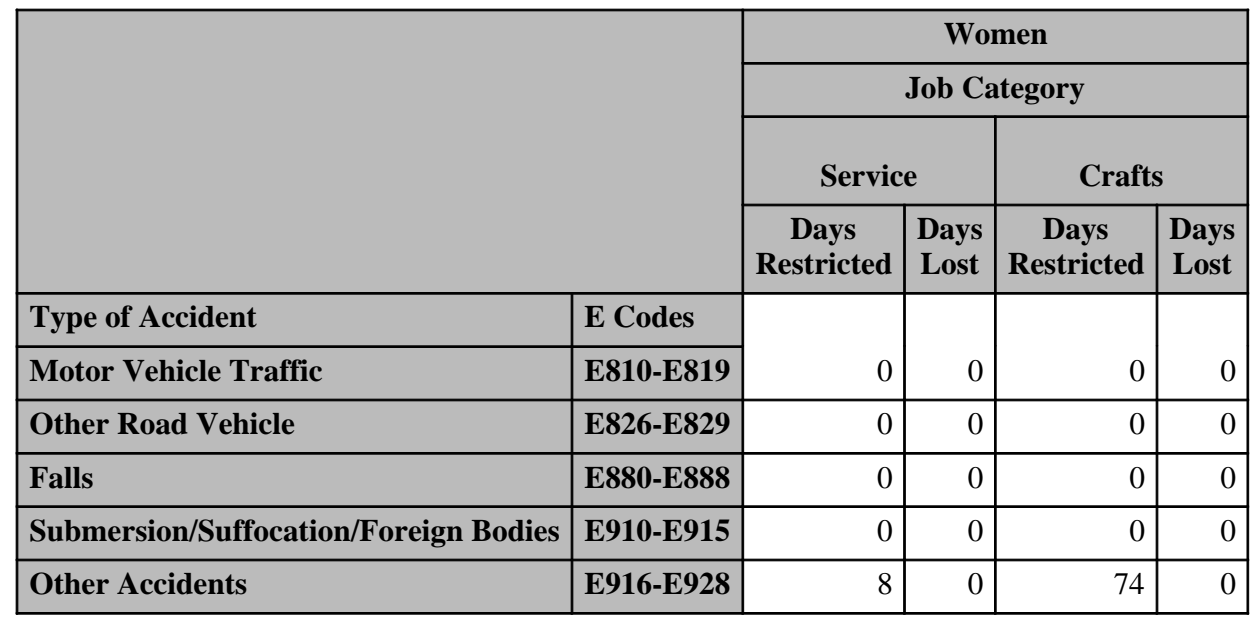

*OSHA events with $>1 \mathrm{E}$ code in the same accident type were counted only once. Only those accident types and gender/job category combinations with at least one occurrence appear in this table. 
Lawrence Livermore National Laboratory 2006

OSHA Data

Appendix V. Number of Workdays Lost or with Restricted Activity in Each Accident Category by Gender and Job Category*

\begin{tabular}{|c|c|c|c|c|c|c|c|}
\hline & & \multicolumn{6}{|c|}{ Men } \\
\hline & & \multicolumn{6}{|c|}{ Job Category } \\
\hline & & \multicolumn{2}{|c|}{ Professional } & \multicolumn{2}{|c|}{$\begin{array}{l}\text { Administrative } \\
\text { Support }\end{array}$} & \multicolumn{2}{|c|}{$\begin{array}{l}\text { Technical } \\
\text { Support }\end{array}$} \\
\hline & & $\begin{array}{c}\text { Days } \\
\text { Restricted }\end{array}$ & $\begin{array}{l}\text { Days } \\
\text { Lost }\end{array}$ & \begin{tabular}{|c|} 
Days \\
Restricted
\end{tabular} & $\begin{array}{l}\text { Days } \\
\text { Lost }\end{array}$ & $\begin{array}{c}\text { Days } \\
\text { Restricted }\end{array}$ & \begin{tabular}{|l} 
Days \\
Lost
\end{tabular} \\
\hline Type of Accident & E Codes & \multirow[b]{2}{*}{0} & \multirow[b]{2}{*}{2} & \multirow[b]{2}{*}{0} & \multirow[b]{2}{*}{0} & \multirow[b]{2}{*}{0} & \multirow[b]{2}{*}{0} \\
\hline Motor Vehicle Traffic & E810-E819 & & & & & & \\
\hline Other Road Vehicle & E826-E829 & 37 & 3 & 0 & 0 & 67 & 22 \\
\hline Falls & E880-E888 & 0 & 0 & 29 & 1 & 0 & 0 \\
\hline Natural/Environmental Factors & E900-E909 & 0 & 0 & 0 & 0 & 8 & 4 \\
\hline Submersion/Suffocation/Foreign Bodies & E910-E915 & 0 & 0 & 78 & 102 & 0 & 0 \\
\hline Other Accidents & E916-E928 & 0 & 0 & 380 & 134 & 530 & 53 \\
\hline Adverse Effect - Medicinal/Biological & E930-E949 & 0 & 0 & 0 & 0 & 0 & 0 \\
\hline
\end{tabular}

\begin{tabular}{|c|c|c|c|c|c|c|c|}
\hline & \multicolumn{6}{|c|}{ Men } \\
\hline & & \multicolumn{6}{|c|}{ Job Category } \\
\hline & & \multicolumn{2}{|c|}{ Service } & \multicolumn{2}{|c|}{$\begin{array}{c}\text { Security and } \\
\text { Fire }\end{array}$} & \multicolumn{2}{|c|}{ Crafts } \\
\hline & & $\begin{array}{c}\text { Days } \\
\text { Restricted }\end{array}$ & $\begin{array}{l}\text { Days } \\
\text { Lost }\end{array}$ & $\begin{array}{c}\text { Days } \\
\text { Restricted }\end{array}$ & $\begin{array}{l}\text { Days } \\
\text { Lost }\end{array}$ & \begin{tabular}{|c|} 
Days \\
Restricted
\end{tabular} & $\begin{array}{l}\text { Days } \\
\text { Lost }\end{array}$ \\
\hline Type of Accident & E Codes & \multirow[b]{2}{*}{0} & \multirow[b]{2}{*}{0} & \multirow[b]{2}{*}{0} & \multirow[b]{2}{*}{0} & \multirow[b]{2}{*}{0} & \multirow[b]{2}{*}{0} \\
\hline Motor Vehicle Traffic & E810-E819 & & & & & & \\
\hline Other Road Vehicle & E826-E829 & 0 & 0 & 0 & 0 & 0 & 0 \\
\hline Falls & E880-E888 & 49 & 87 & 0 & 180 & 33 & 0 \\
\hline Natural/Environmental Factors & E900-E909 & 0 & 13 & 0 & 0 & 0 & 0 \\
\hline Submersion/Suffocation/Foreign Bodies & E910-E915 & 0 & 0 & 0 & 0 & 0 & 0 \\
\hline Other Accidents & E916-E928 & 441 & 139 & 927 & 105 & 387 & 240 \\
\hline Adverse Effect - Medicinal/Biological & E930-E949 & 0 & 0 & 0 & 0 & 0 & 0 \\
\hline
\end{tabular}

*OSHA events with >1 E code in the same accident type were counted only once. Only those accident types and gender/job category combinations with at least one occurrence appear in this table. 
Lawrence Livermore National Laboratory 2006

OSHA Data

Appendix W. Age-Adjusted OSHA Illness and Injury Rates by Diagnostic Category

Part 1. Men

\begin{tabular}{|c|c|c|c|c|c|}
\hline Category of Diagnoses & ICD-9-CM Code & $\begin{array}{c}\text { Number of } \\
\text { Diagnoses }\end{array}$ & $\begin{array}{c}\text { Age-Adjusted } \\
\text { Rate per 1000* }\end{array}$ & \begin{tabular}{|c|} 
Lower $95 \%$ \\
Confidence \\
Limit per 1000
\end{tabular} & \begin{tabular}{|c|} 
Upper $95 \%$ \\
Confidence \\
Limit per 1000
\end{tabular} \\
\hline Infections/Parasites & $001-139$ & 2 & 0.5 & 0.1 & 1.9 \\
\hline Cancer & $140-208,230-234$ & 0 & 0 & 0 & 0 \\
\hline Benign Growths & $210-229,235-239$ & 0 & 0 & 0 & 0 \\
\hline Endocrine/Metabolic & $240-279$ & 0 & 0 & 0 & 0 \\
\hline Blood & $280-289$ & 0 & 0 & 0 & 0 \\
\hline Mental & $290-319$ & 0 & 0 & 0 & 0 \\
\hline Nervous System & $320-389$ & 3 & 0.8 & 0.2 & 2.8 \\
\hline -Disorders of Peripheral NS & $350-359$ & 1 & 0.2 & 0.0 & 1.7 \\
\hline -Disorders of Eye & $360-379$ & 1 & 0.4 & 0.1 & 3.2 \\
\hline -Disorders of Ear \& Mastoid & $380-389$ & 1 & 0.1 & 0.0 & $\overline{0.6}$ \\
\hline Heart/Circulatory & $390-459$ & 0 & 0 & 0 & 0 \\
\hline Respiratory & $460-519$ & 2 & 0.2 & 0.1 & 0.9 \\
\hline -Chronic Obstructive Diseases & $490-496$ & 0 & 0 & 0 & 0 \\
\hline -Lung Disease from External Agents & $500-508$ & 0 & 0 & 0 & 0 \\
\hline Digestive & $520-579$ & 0 & 0 & 0 & 0 \\
\hline -Hernias & $550-553$ & 0 & 0 & 0 & 0 \\
\hline Genitourinary & $580-629$ & 0 & 0 & 0 & 0 \\
\hline Skin & $680-709$ & 3 & 0.8 & 0.2 & 2.8 \\
\hline Musculoskeletal & $710-739$ & 56 & 7.1 & 5.2 & 9.6 \\
\hline -Arthropathies & $710-719$ & 26 & 2.7 & 1.8 & 4.0 \\
\hline -Dorsopathies & $720-724$ & 17 & 2.8 & 1.6 & 5.0 \\
\hline -Rheumatism, Excluding Back & $725-729$ & 13 & 1.6 & 0.9 & 2.9 \\
\hline Unspecified Symptoms & $780-799$ & 13 & 1.8 & 1.0 & 3.4 \\
\hline Injury & $800-999$ & 104 & 14.5 & 11.6 & 18.3 \\
\hline -Fractures - Skull, Neck, Trunk & $800-809$ & 1 & 0.1 & 0.0 & $\overline{0.6}$ \\
\hline -Fractures - Upper Limb & $810-819$ & 9 & 1.4 & 0.7 & 2.8 \\
\hline -Fractures - Lower Limb & $820-829$ & 1 & 0.1 & 0.0 & 0.6 \\
\hline -Dislocations & $830-839$ & 0 & 0 & 0 & 0 \\
\hline -Back Sprains \& Strains & $846-847$ & 14 & 2.9 & 1.5 & 5.3 \\
\hline -Other Sprains \& Strains & $840-845,848$ & 33 & 3.7 & 2.6 & 5.3 \\
\hline -Open Wounds - Head, Neck, Trunk & $870-879$ & 2 & 0.4 & 0.1 & 1.6 \\
\hline -Open Wounds - Upper Limb & $880-887$ & 11 & 1.4 & 0.7 & 2.5 \\
\hline -Open Wounds - Lower Limb & $890-897$ & 1 & 0.1 & 0.0 & 0.9 \\
\hline -Superficial Injuries & $910-919$ & 2 & 0.2 & 0.0 & 0.7 \\
\hline -Bruises & $920-924$ & 9 & 1.3 & 0.6 & 3.0 \\
\hline -Foreign Bodies Entering Orifice & $930-939$ & 2 & 0.2 & 0.0 & 0.7 \\
\hline -Burns & $940-949$ & 2 & 0.2 & 0.0 & 0.7 \\
\hline
\end{tabular}

*Standardized to age distribution of 2000 U.S. population. 
Lawrence Livermore National Laboratory 2006

OSHA Data

Appendix W. Age-Adjusted OSHA Illness and Injury Rates by Diagnostic Category

\begin{tabular}{|c|c|c|c|c|c|}
\hline Category of Diagnoses & ICD-9-CM Code & $\begin{array}{l}\text { Number of } \\
\text { Diagnoses }\end{array}$ & $\begin{array}{l}\text { Age-Adjusted } \\
\text { Rate per } 1000 *\end{array}$ & $\begin{array}{c}\text { Lower } 95 \% \\
\text { Confidence } \\
\text { Limit per } 1000\end{array}$ & $\begin{array}{c}\text { Upper } 95 \% \\
\text { Confidence } \\
\text { Limit per } 1000\end{array}$ \\
\hline $\begin{array}{r}\text {-Adverse Reactions to Nonmedical } \\
\text { Substances or External Causes }\end{array}$ & $980-995$ & 1 & 0.2 & 0.0 & 1.7 \\
\hline -All Other Injuries & \begin{tabular}{|c|}
$850-854,860-869,900-909$ \\
$925-929,950-979,996-999$
\end{tabular} & 16 & 2.5 & 1.4 & 4.7 \\
\hline Health Status/Health Service Contact & V01-V82 & 0 & 0 & 0 & 0 \\
\hline Total & & 183 & 25.7 & 21.6 & 30.5 \\
\hline
\end{tabular}

\section{Part 2. Women}

\begin{tabular}{|c|c|c|c|c|c|}
\hline Category of Diagnoses & ICD-9-CM Code & $\begin{array}{l}\text { Number of } \\
\text { Diagnoses }\end{array}$ & $\begin{array}{l}\text { Age-Adjusted } \\
\text { Rate per } 1000 *\end{array}$ & \begin{tabular}{|c|} 
Lower $95 \%$ \\
Confidence \\
Limit per 1000
\end{tabular} & $\begin{array}{c}\text { Upper } 95 \% \\
\text { Confidence } \\
\text { Limit per } 1000\end{array}$ \\
\hline Infections/Parasites & $001-139$ & 1 & 0.2 & 0.0 & 1.4 \\
\hline Cancer & $140-208,230-234$ & 0 & 0 & 0 & 0 \\
\hline Benign Growths & $210-229,235-239$ & 0 & 0 & 0 & 0 \\
\hline Endocrine/Metabolic & $240-279$ & 0 & 0 & 0 & 0 \\
\hline Blood & $280-289$ & 0 & 0 & 0 & 0 \\
\hline Mental & $290-319$ & 0 & 0 & 0 & 0 \\
\hline Nervous System & $320-389$ & 4 & 1.6 & 0.5 & 5.0 \\
\hline -Disorders of Peripheral NS & $350-359$ & 4 & 1.6 & 0.5 & 5.0 \\
\hline -Disorders of Eye & $360-379$ & 0 & 0 & 0 & 0 \\
\hline -Disorders of Ear \& Mastoid & $380-389$ & 0 & 0 & 0 & 0 \\
\hline Heart/Circulatory & $390-459$ & 0 & 0 & 0 & 0 \\
\hline Respiratory & $460-519$ & 0 & 0 & 0 & 0 \\
\hline -Chronic Obstructive Diseases & $490-496$ & 0 & 0 & 0 & 0 \\
\hline -Lung Disease from External Agents & $500-508$ & 0 & 0 & 0 & 0 \\
\hline Digestive & $520-579$ & 0 & 0 & 0 & 0 \\
\hline -Hernias & $550-553$ & 0 & 0 & 0 & 0 \\
\hline Genitourinary & $580-629$ & 0 & 0 & 0 & 0 \\
\hline Skin & $680-709$ & 1 & 0.2 & 0.0 & 1.4 \\
\hline Musculoskeletal & $710-739$ & 62 & 25.6 & 19.3 & 34.1 \\
\hline -Arthropathies & $710-719$ & 39 & 17.9 & 12.5 & 25.5 \\
\hline -Dorsopathies & $720-724$ & 8 & 2.3 & 1.0 & 5.2 \\
\hline -Rheumatism, Excluding Back & $725-729$ & 15 & 5.5 & 3.1 & 9.7 \\
\hline Unspecified Symptoms & $780-799$ & 12 & 2.9 & 1.6 & 5.2 \\
\hline Injury & $800-999$ & 44 & 15.3 & 11.0 & 21.5 \\
\hline -Fractures - Skull, Neck, Trunk & $800-809$ & 0 & 0 & 0 & 0 \\
\hline -Fractures - Upper Limb & $810-819$ & 1 & 0.2 & 0.0 & 1.8 \\
\hline -Fractures - Lower Limb & $820-829$ & 1 & 0.5 & 0.1 & 3.8 \\
\hline -Dislocations & $830-839$ & 0 & 0 & 0 & 0 \\
\hline
\end{tabular}

*Standardized to age distribution of 2000 U.S. population. 
Lawrence Livermore National Laboratory 2006

OSHA Data

Appendix W. Age-Adjusted OSHA Illness and Injury Rates by Diagnostic Category

\begin{tabular}{|c|c|c|c|c|c|}
\hline Category of Diagnoses & ICD-9-CM Code & $\begin{array}{l}\text { Number of } \\
\text { Diagnoses }\end{array}$ & $\begin{array}{l}\text { Age-Adjusted } \\
\text { Rate per } 1000^{*}\end{array}$ & \begin{tabular}{|c|} 
Lower $95 \%$ \\
Confidence \\
Limit per 1000
\end{tabular} & $\begin{array}{c}\text { Upper } 95 \% \\
\text { Confidence } \\
\text { Limit per } 1000\end{array}$ \\
\hline -Back Sprains \& Strains & $846-847$ & 7 & 1.8 & 0.8 & 4.0 \\
\hline -Other Sprains \& Strains & $840-845,848$ & 6 & 1.8 & 0.7 & 4.8 \\
\hline -Open Wounds - Head, Neck, Trunk & $870-879$ & 1 & 0.2 & 0.0 & 1.4 \\
\hline -Open Wounds - Upper Limb & $880-887$ & 0 & 0 & 0 & 0 \\
\hline -Open Wounds - Lower Limb & $890-897$ & 0 & 0 & 0 & 0 \\
\hline -Superficial Injuries & $910-919$ & 7 & 2.3 & 1.0 & 5.1 \\
\hline -Bruises & $920-924$ & 4 & 0.8 & 0.3 & 2.3 \\
\hline -Foreign Bodies Entering Orifice & $930-939$ & 1 & 0.5 & 0.1 & 3.5 \\
\hline -Burns & $940-949$ & 0 & 0 & 0 & 0 \\
\hline $\begin{array}{r}\text {-Adverse Reactions to Nonmedical } \\
\text { Substances or External Causes }\end{array}$ & $980-995$ & 0 & 0 & 0 & 0 \\
\hline -All Other Injuries & \begin{tabular}{|c|}
$850-854,860-869,900-909$ \\
$925-929,950-979,996-999$
\end{tabular} & 16 & 7.1 & 4.1 & 12.2 \\
\hline Health Status/Health Service Contact & V01-V82 & 0 & 0 & 0 & 0 \\
\hline Total & & 124 & 45.9 & 37.5 & 56.2 \\
\hline
\end{tabular}

Part 3. Men and Women

\begin{tabular}{|c|c|c|c|c|c|}
\hline Category of Diagnoses & ICD-9-CM Code & $\begin{array}{l}\text { Number of } \\
\text { Diagnoses }\end{array}$ & $\begin{array}{l}\text { Age-Adjusted } \\
\text { Rate per } 1000^{*}\end{array}$ & \begin{tabular}{|c|} 
Lower $95 \%$ \\
Confidence \\
Limit per 1000
\end{tabular} & $\begin{array}{c}\text { Upper } 95 \% \\
\text { Confidence } \\
\text { Limit per } 1000\end{array}$ \\
\hline Infections/Parasites & $001-139$ & 3 & 0.4 & 0.1 & 1.3 \\
\hline Cancer & $140-208,230-234$ & 0 & 0 & 0 & 0 \\
\hline Benign Growths & $210-229,235-239$ & 0 & 0 & 0 & 0 \\
\hline Endocrine/Metabolic & $240-279$ & 0 & 0 & 0 & 0 \\
\hline Blood & $280-289$ & 0 & 0 & 0 & 0 \\
\hline Mental & $290-319$ & 0 & 0 & 0 & 0 \\
\hline Nervous System & $320-389$ & 7 & 1.0 & 0.4 & 2.5 \\
\hline -Disorders of Peripheral NS & $350-359$ & 5 & 0.7 & 0.3 & 2.0 \\
\hline -Disorders of Eye & $360-379$ & 1 & 0.3 & 0.0 & 2.0 \\
\hline -Disorders of Ear \& Mastoid & $380-389$ & 1 & 0.1 & 0.0 & 0.4 \\
\hline Heart/Circulatory & $390-459$ & 0 & 0 & 0 & 0 \\
\hline Respiratory & $460-519$ & 2 & 0.1 & 0.0 & 0.6 \\
\hline -Chronic Obstructive Diseases & $490-496$ & 0 & 0 & 0 & 0 \\
\hline -Lung Disease from External Agents & $500-508$ & 0 & 0 & 0 & 0 \\
\hline Digestive & $520-579$ & 0 & 0 & 0 & 0 \\
\hline -Hernias & $550-553$ & 0 & 0 & 0 & 0 \\
\hline Genitourinary & $580-629$ & 0 & 0 & 0 & 0 \\
\hline Skin & $680-709$ & 4 & 0.6 & 0.2 & 1.8 \\
\hline Musculoskeletal & $710-739$ & 118 & 13.5 & 10.9 & 16.8 \\
\hline
\end{tabular}

*Standardized to age distribution of 2000 U.S. population. 
Lawrence Livermore National Laboratory 2006

OSHA Data

Appendix W. Age-Adjusted OSHA IIlness and Injury Rates by Diagnostic Category

\begin{tabular}{|c|c|c|c|c|c|}
\hline Category of Diagnoses & ICD-9-CM Code & $\begin{array}{l}\text { Number of } \\
\text { Diagnoses }\end{array}$ & $\begin{array}{l}\text { Age-Adjusted } \\
\text { Rate per } 1000^{*}\end{array}$ & \begin{tabular}{|c|} 
Lower $95 \%$ \\
Confidence \\
Limit per 1000
\end{tabular} & \begin{tabular}{|c|} 
Upper $95 \%$ \\
Confidence \\
Limit per 1000
\end{tabular} \\
\hline -Arthropathies & $710-719$ & 65 & 8.0 & 5.9 & 10.7 \\
\hline -Dorsopathies & $720-724$ & 25 & 2.6 & 1.6 & 4.2 \\
\hline -Rheumatism, Excluding Back & $725-729$ & 28 & 2.9 & 1.9 & 4.5 \\
\hline Unspecified Symptoms & $780-799$ & 25 & 2.2 & 1.4 & 3.3 \\
\hline Injury & $800-999$ & 148 & 14.6 & 12.1 & 17.7 \\
\hline -Fractures - Skull, Neck, Trunk & $800-809$ & 1 & 0.1 & 0.0 & 0.4 \\
\hline -Fractures - Upper Limb & $810-819$ & 10 & 1.0 & 0.5 & 2.0 \\
\hline -Fractures - Lower Limb & $820-829$ & 2 & 0.2 & 0.0 & 0.7 \\
\hline -Dislocations & $830-839$ & 0 & 0 & 0 & 0 \\
\hline -Back Sprains \& Strains & $846-847$ & 21 & 2.4 & 1.5 & 4.0 \\
\hline -Other Sprains \& Strains & $840-845,848$ & 39 & 3.2 & 2.2 & 4.5 \\
\hline -Open Wounds - Head, Neck, Trunk & $870-879$ & 3 & 0.3 & 0.1 & 1.0 \\
\hline -Open Wounds - Upper Limb & $880-887$ & 11 & 0.9 & 0.5 & 1.7 \\
\hline -Open Wounds - Lower Limb & $890-897$ & 1 & 0.1 & 0.0 & 0.6 \\
\hline -Superficial Injuries & $910-919$ & 9 & 0.8 & 0.4 & 1.7 \\
\hline -Bruises & $920-924$ & 13 & 1.2 & 0.6 & 2.2 \\
\hline -Foreign Bodies Entering Orifice & $930-939$ & 3 & 0.3 & 0.1 & 1.0 \\
\hline -Burns & 940-949 & 2 & 0.1 & 0.0 & 0.5 \\
\hline $\begin{array}{r}\text {-Adverse Reactions to Nonmedical } \\
\text { Substances or External Causes }\end{array}$ & $980-995$ & 1 & 0.2 & 0.0 & 1.1 \\
\hline -All Other Injuries & \begin{tabular}{|c|}
$850-854,860-869,900-909$ \\
$925-929,950-979,996-999$
\end{tabular} & 32 & 4.0 & 2.6 & 6.0 \\
\hline Health Status/Health Service Contact & V01-V82 & 0 & 0 & 0 & 0 \\
\hline Total & & 307 & 32.5 & 28.4 & 37.1 \\
\hline
\end{tabular}

*Standardized to age distribution of 2000 U.S. population. 